\title{
Boundaries of systolic groups
}

\author{
DAMIAN OSAJDA \\ PIOTR PRZYTYCKI
}

\begin{abstract}
For all systolic groups we construct boundaries which are $E Z$-structures. This implies the Novikov conjecture for torsion-free systolic groups. The boundary is constructed via a system of distinguished geodesics in a systolic complex, which we prove to have coarsely similar properties to geodesics in CAT(0) spaces.
\end{abstract}

20F65, 20F67; 20F69

\section{Introduction}

There are many notions of boundaries of groups used for various purposes. In this paper we focus on the notions of $Z$-structure and $E Z$-structure introduced by Bestvina [3] and studied eg by Dranishnikov [12] and Farrell-Lafont [16]. Our main result is the following.

Theorem A (Theorem 6.3) Let a group $G$ act geometrically by simplicial automorphisms on a systolic complex $X$. Then there exists a compactification $\bar{X}=X \cup \partial X$ of $X$ satisfying the following:

(1) $\bar{X}$ is a Euclidean retract (ER).

(2) $\partial X$ is a $Z$-set in $\bar{X}$.

(3) For every compact set $K \subset X,(g K)_{g \in G}$ is a null sequence.

(4) The action of $G$ on $X$ extends to an action by homeomorphisms of $G$ on $\bar{X}$.

A group $G$ as in Theorem A is called a systolic group. It is a group acting geometrically (ie cocompactly and properly discontinuously) by simplicial automorphisms on a systolic complex — contractible simplicial complex satisfying some local combinatorial conditions. Systolic complexes were introduced by Chepoi [8] (under the name of bridged complexes) and, independently, by Januszkiewicz-Światkowski [19] and by Haglund [17] (in Section 2 we give some background on them). Systolic complexes (groups) have many properties of nonpositively curved spaces (groups). There are systolic complexes that are not $\mathrm{CAT}(0)$ when equipped with the path metric in which 
every simplex is isometric to the standard Euclidean simplex. On the other hand, there are systolic groups that are not hyperbolic, eg $\mathbb{Z}^{2}$. Summarizing, the systolic setting does not reduce to the CAT(0) or to the hyperbolic one. Systolic groups admit various combinatorial constructions (see Haglund [17], Januszkiewicz-Świątkowski [19] and Arzhantseva et al [1]) with unexpected properties (see Januszkiewicz-Świątkowski [20] and Arzhantseva et al [1]). We also believe that eventually both systolic complexes and $\mathrm{CAT}(0)$ cubical ones will be placed among a wider family of combinatorially nonpositively curved contractible cell complexes.

Here we give the other definitions that appear in the statement of Theorem A. A compact space is a Euclidean retract (or ER) if it can be embedded in some Euclidean space as its retract. A closed subset $Z$ of a Euclidean retract $Y$ is called a $Z-$ set if for every open set $U \subset Y$, the inclusion $U \backslash Z \hookrightarrow U$ is a homotopy equivalence. A sequence $\left(K_{i}\right)_{i=1}^{\infty}$ of subsets of a topological space $Y$ is called a null sequence if for every open cover $\mathcal{U}=\left\{U_{i}\right\}_{i \in I}$ of $Y$ all but finitely many $K_{i}$ are $\mathcal{U}$-small, ie for all but finitely many $j$ there exist $i(j)$ such that $K_{j} \subset U_{i(j)}$.

Conditions (1), (2) and (3) of Theorem A mean (following Bestvina [3], where only free actions are considered, and Dranishnikov [12]) that any systolic group $G$ admits a $Z$-structure $(\bar{X}, \partial X)$. The notion of an $E Z$-structure, ie a $Z$-structure with the additional property (4) was explored by Farrell-Lafont [16] (in the case of a free action). Bestvina [3] showed that some local homological invariants of the boundary $\partial X$ are related to cohomological invariants of the group. In particular, the dimension of the boundary is an invariant of the group ie it does not depend on the $Z$-structure we choose. This was generalized by Dranishnikov [12] to the case of geometric actions. We emphasize that the homeomorphism type of the boundary is not a group invariant (but the shape is an invariant; see Bestvina [3]). This was proved by Croke-Kleiner [10] in the context of visual boundaries of CAT(0) spaces.

Carlsson-Pedersen [7] and Farrell-Lafont [16] proved that existence of an $E Z$-structure on a torsion-free group $G$ implies that the Novikov conjecture is true for $G$. Thus, by Theorem A, we get the following.

\section{Corollary Torsion-free systolic groups satisfy the Novikov conjecture.}

Bartels-Lück [2] prove the Borel conjecture (which in particular implies the Novikov conjecture) for a class $\mathcal{B}$ of groups which includes $\mathrm{CAT}(0)$ groups and hyperbolic groups and is closed under some elementary operations. All systolic groups known to us belong to the class $\mathcal{B}$. However it is not likely that all systolic groups belong to $\mathcal{B}$. There are only a few classes of groups for which a $Z$-structure $(\bar{X}, \partial X)$ has been found (and even fewer for which an $E Z$-structure is known). The most important examples 
are: hyperbolic groups (see Bestvina-Mess [4]) —where $X$ is the Rips complex and $\partial X$ is the Gromov boundary; CAT(0) groups — where $X$ is a CAT(0) space and $\partial X$ is the visual boundary of $X$; relatively hyperbolic groups whose parabolic subgroups admit a $Z$-structure (see Dahmani [11]). Bestvina [3] asked whether every group $G$ with finite $K(G, 1)$ has a $Z$-structure.

The question whether every systolic group has an $E Z$-structure was posed by Januszkiewicz and Świątkowski in 2004. Theorem A answers affirmatively this question.

We hope that, similarly to the hyperbolic and $\mathrm{CAT}(0)$ cases, our boundaries will be also useful for purposes other than the ones mentioned above. In particular we think that splittings of systolic groups can be recognized through the topology of the boundary, as in eg Bowditch [5] and Papasoglu-Swenson [22]. Studying more refined structures on the boundary could help in obtaining rigidity results for some systolic groups.

The essential point of our construction is the choice of the system of good geodesics (derived from the system of Euclidean geodesics, the distinction is not important at this moment), which is coarsely closed under taking subsegments (Theorem B below), and which satisfies coarsely a weak form of CAT(0) condition (Theorem C below).

Recall that Januszkiewicz-Świątkowski [19] considered a system of directed geodesics in a systolic complex (cf Definition 2.11). One may try to define the boundary of a systolic complex by taking the inverse limit of the following system. Consider the sequence of combinatorial spheres around a fixed vertex $O$ and projections from larger to smaller spheres along the directed geodesics terminating at $O$. Unfortunately, the inverse limit of this system does not satisfy, in general, property (3) of Theorem A. Property (3) fails, for example, already for the flat systolic plane (cf Definition 7.1).

Hence, instead of using directed geodesics, we introduce Euclidean geodesics, which behave like CAT(0) geodesics with respect to the flat subcomplexes of a systolic complex. To define the Euclidean geodesic between two vertices, say $s, t$, in a systolic complex, we consider the loop obtained by concatenating the two directed geodesics joining $s$ to $t$ and $t$ to $s$. Then we span a minimal surface $S$ on this loop. (We make use of minimal surface theory developed by Elsner [15]. To obtain some uniqueness properties on $S$ we complement Elsner's theory with our results on layers, which span the union of all 1 -skeleton geodesics between $t$ and $s$.) The surface $S$ is isometric to a contractible subcomplex of the flat systolic plane and hence has a natural structure of a CAT(0) space. The Euclidean geodesic is defined as a sequence of simplices in $S$, which runs near the CAT( 0$)$ geodesic between $s$ and $t$.

Now we pass to the more technical part of the exposition. Formally, the Euclidean geodesic is defined for a pair of simplices $\sigma, \tau$ in a systolic complex, which satisfies 
$\sigma \subset S_{n}(\tau), \tau \subset S_{n}(\sigma)$ for some $n \geq 0$ (where $S_{n}(\sigma)$ denotes the combinatorial sphere of radius $n$ around $\sigma$, cf Definition 2.4). The Euclidean geodesic is a certain sequence of simplices $\delta_{k}$, where $0 \leq k \leq n$, such that $\delta_{0}=\sigma, \delta_{n}=\tau$, and $\delta_{k} \subset$ $S_{1}\left(\delta_{k+1}\right), \delta_{k+1} \subset S_{1}\left(\delta_{k}\right)$ for $0 \leq k<n$ (cf Lemma 9.15(i)). The two most significant features of Euclidean geodesics are given by the following.

Theorem B (Theorem 12.2) Let $\sigma, \tau$ be simplices of a systolic complex $X$, such that for some natural $n$ we have $\sigma \subset S_{n}(\tau), \tau \subset S_{n}(\sigma)$. Let $\left(\delta_{k}\right)_{k=0}^{n}$ be the Euclidean geodesic between $\sigma$ and $\tau$. Take some $0 \leq l<m \leq n$ and let $\left(r_{k}\right)_{k=l}^{m}$ be a $1-$ skeleton geodesic such that $r_{k} \in \delta_{k}$ for $l \leq k \leq m$. Consider the simplices $\tilde{\delta}_{l}=$ $r_{l}, \widetilde{\delta}_{l+1}, \ldots, \tilde{\delta}_{m}=r_{m}$ of the Euclidean geodesic between vertices $r_{l}$ and $r_{m}$. Then for each $l \leq k \leq m$ we have $\left|\delta_{k}, \widetilde{\delta}_{k}\right| \leq C$, where $C$ is a universal constant.

Theorem C (Theorem 13.1) Let $s, s^{\prime}, t$ be vertices in a systolic complex $X$ such that $|s t|=n,\left|s^{\prime} t\right|=n^{\prime}$. Let $\left(r_{k}\right)_{k=0}^{n},\left(r_{k}^{\prime}\right)_{k=0}^{n^{\prime}}$ be 1-skeleton geodesics such that $r_{k} \in$ $\delta_{k}, r_{k}^{\prime} \in \delta_{k}^{\prime}$, where $\left(\delta_{k}\right),\left(\delta_{k}^{\prime}\right)$ are Euclidean geodesics for $t, s$ and for $t, s^{\prime}$ respectively. Then for all $0 \leq c \leq 1$ we have $\left|r_{\lfloor c n\rfloor} r_{\left\lfloor c n^{\prime}\right\rfloor}^{\prime}\right| \leq c\left|s s^{\prime}\right|+C$, where $C$ is a universal constant.

The article is organized as follows. It consists of an introductory part (Sections 1-2), two main parts (Sections 3-6 and Sections 7-13) which can be read independently and a concluding Section 14.

In Section 2 we give a brief introduction to systolic complexes.

In the first part, assuming we have defined Euclidean geodesics satisfying Theorem B and Theorem C, we define the boundary: In Section 3 we define the boundary as a set of equivalence classes of good geodesic rays. Then we define topology on the compactification obtained by adjoining the boundary (Section 4) and we show its compactness and finite dimensionality (Section 5). Finally, in Section 6, we prove Theorem A - the main result of the paper.

In the second part of the article we define Euclidean geodesics and establish Theorem B and Theorem C: In Section 7 we recall Elsner's results on minimal surfaces. In Section 8 we study layers, whose union contains all geodesics between given vertices. We define Euclidean geodesics in Section 9.

In the next two sections we prove Theorem 10.1 which is a weak version of Theorem B (though with a better constant). Apart from the definitions these sections can be skipped by a hurried reader. We decided to include them since this way of obtaining (the weak version of) Theorem B is straightforward in opposition to the strategy in 
Section 12, which is designed to obtain Theorem C. In Section 10 we study the position of directed geodesics between two simplices of a given Euclidean geodesic with respect to the minimal surface appearing in its construction. Then we verify Theorem 10.1 in Section 11 by studying CAT( 0 ) geometry of minimal surfaces.

The last two sections are devoted to the proofs of Theorem B and Theorem C: In Section 12 we prove (in a technically cumbersome manner) the powerful Proposition 12.1 linked with CAT(0) properties of the triangles, whose two sides are Euclidean geodesics. Proposition 12.1 easily implies Theorem B, but its main application comes in Section 13, where we use it to derive Theorem C.

We conclude with announcing some further results for which we do not provide proofs in Section 14.

Acknowledgments We are grateful to Tadeusz Januszkiewicz and Jacek Świątkowski for discussions and to Mladen Bestvina for encouragement. We thank the Mathematical Sciences Research Institute and the Institut des Hautes Études Scientifiques for its hospitality during the preparation of this article.

The first author was partially supported by MNiSW grant N201 012 32/0718. This research was supported by a Marie Curie European Reintegration Grant within the 6th European Community Framework Programme. The second author was partially supported by MNiSW grant N201 003 32/0070, MNiSW grant N201 012 32/0718 and the Foundation for Polish Science.

\section{Systolic complexes}

In this section we recall (from Januszkiewicz-Świątkowski [19; 20] and HaglundŚwiatkowski [18]) the definition and basic properties of systolic complexes and groups.

Definition 2.1 A subcomplex $K$ of a simplicial complex $X$ is called full in $X$ if any simplex of $X$ spanned by vertices of $K$ is a simplex of $K$. The span of a subcomplex $K \subset X$ is the smallest full subcomplex of $X$ containing $K$. We denote it by $\operatorname{span}(K)$. A simplicial complex $X$ is called flag if any set of vertices, which are pairwise connected by edges of $X$, spans a simplex in $X$. A simplicial complex $X$ is called $k$-large, for $\infty \geq k \geq 4$, if $X$ is flag and there are no embedded cycles of length less than $k$, which are full subcomplexes of $X$ (ie $X$ is flag and every simplicial cycle of length at least 4 and less than $k$ "has a diagonal").

Definition 2.2 A simplicial complex $X$ is called systolic if it is connected, simply connected and links of all simplices in $X$ are 6-large. A group $\Gamma$ is called systolic 
if it acts cocompactly and properly (ie geometrically) by simplicial automorphisms on a systolic complex $X$. (Properly means $X$ is locally finite and for each compact subcomplex $K \subset X$ the set of $\gamma \in \Gamma$ such that $\gamma(K) \cap K \neq \varnothing$ is finite.)

Recall a result of Januszkiewicz-Świątkowski [19, Proposition 1.4 ], that systolic complexes are themselves 6-large. In particular they are flag. Moreover, we have the following.

Theorem 2.3 [19, Theorem 4.1(1)] Finite dimensional systolic complexes are contractible.

Now we briefly treat the definitions and facts concerning convexity.

Definition 2.4 For every pair of subcomplexes (usually vertices) $A, B$ in a simplicial complex $X$ denote by $|A, B|(|a b|$ for vertices $a, b)$ the combinatorial distance between $A^{(0)}, B^{(0)}$ in $X^{(1)}$, the 1 -skeleton of $X$ (ie the minimal number of edges in a simplicial path connecting both sets). A subcomplex $K$ of a simplicial complex $X$ is called 3-convex if it is a full subcomplex of $X$ and for every pair of edges $a b, b c$ such that $a, c \in K,|a c|=2$, we have $b \in K$. A subcomplex $K$ of a systolic complex $X$ is called convex if it is connected and links of all simplices in $K$ are 3-convex subcomplexes of links of those simplices in $X$.

Januszkiewicz-Świątkowski [19, Lemma 7.2] conclude that convex subcomplexes of a systolic complex $X$ are full and 3-convex in $X$, and systolic themselves, hence contractible by Theorem 2.3. The intersection of a family of convex subcomplexes is convex. For a subcomplex $Y \subset X, n \geq 0$, the combinatorial ball $B_{n}(Y)$ of radius $n$ around $Y$ is the span of $\left\{p \in X^{(0)}:|p, Y| \leq n\right\}$. (Similarly $S_{n}(Y)=\operatorname{span}\left\{p \in X^{(0)}\right.$ : $|p, Y|=n\}$.) If $Y$ is convex (in particular, if $Y$ is a simplex) then $B_{n}(Y)$ is also convex, as proved by Januszkiewicz-Świątkowski [19, Corollary 7.5]. Combining this with previous remarks we record:

Corollary 2.5 In systolic complexes, balls around simplices are contractible.

Haglund-Świątkowski prove the following.

Proposition 2.6 [18, Proposition 4.9] A full subcomplex $Y$ of a systolic complex $X$ is convex if and only if $Y^{(1)}$ is geodesically convex in $X^{(1)}$ (ie if all geodesics in $X^{(1)}$ joining vertices of $Y$ lie in $\left.Y^{(1)}\right)$.

We record:

Corollary 2.7 In systolic complexes balls around simplices are geodesically convex. 
We will need a crucial "projection lemma". The residue of a simplex $\sigma$ in $X$ is the union of all simplices in $X$, which contain $\sigma$.

Lemma 2.8 [19, Lemma 7.7] Let $Y$ be a convex subcomplex of a systolic complex $X$ and let $\sigma$ be a simplex in $S_{1}(Y)$. Then the intersection of the residue of $\sigma$ and of the complex $Y$ is a simplex (in particular it is nonempty).

Definition 2.9 The simplex as in Lemma 2.8 is called the projection of $\sigma$ onto $Y$.

The following lemma immediately follows from Definition 2.9.

Lemma 2.10 Let $\sigma \subset \widetilde{\sigma}$ be simplices in $S_{1}(Y)$ for some convex $Y$ and let $\pi, \tilde{\pi}$ be their projections onto $Y$. Then $\tilde{\pi} \subset \pi$.

Definition 2.11 For a pair of vertices $v, w$ with $|v w|=n$ in a systolic complex $X$ we define inductively the sequence of simplices $\sigma_{0}=v, \sigma_{1}, \ldots, \sigma_{n}=w$ as follows. Take $\sigma_{i}$ equal to the projection of $\sigma_{i-1}$ onto $B_{n-i}(w)$ for $i=1, \ldots, n-1, n$. The sequence $\left(\sigma_{i}\right)_{i=0}^{n}$ is called the directed geodesic from $v$ to $w$ (this notion is introduced and studied by Januszkiewicz-Światkowski [19]).

We can extend this construction to any pair $\left(\sigma_{0}, W\right)$, where $W$ is a convex subcomplex of $X$ and $\sigma_{0}$ is a simplex. Namely, if for some $n$ we have $\sigma_{0} \subset S_{n}(W)$ then take $\sigma_{i}$ to be the projection of $\sigma_{i-1}$ onto $B_{n-i}(W)$. If $\sigma_{0}$ intersects both $S_{n}(W)$ and $S_{n-1}(W)$ then take $\sigma_{1}=\sigma_{0} \cap S_{n-1}(W)$ and then proceed as previously. We call the final $\sigma_{n} \subset W$ the projection of $\sigma_{0}$ onto $W$. Note that this coincides with Definition 2.9. Observe that if $\sigma_{0} \subset W$ then the projection of $\sigma_{0}$ onto $W$ is equal to $\sigma_{0}$.

Finally, recall a powerful observation.

Lemma 2.12 [20, Lemma 4.4] Every full subcomplex of a systolic complex is aspherical.

\section{Definition of the boundary}

Let $X$ be a systolic complex. In this section we give two equivalent definitions of the boundary of $X$ as a set. We use the notion of Euclidean geodesics which will be introduced in Section 9, but actually we need only its features given by Theorem B and Theorem C. Thus, it is enough to read Sections 1-2 to follow the first part of the article (Sections 3-6). Let $C$ be a natural number, which is a universal constant satisfying assertions of both Theorem B and Theorem C. 
Remark 3.1 Let $\left(\delta_{i}\right)_{i=0}^{n}$ be a Euclidean geodesic and let $v_{k}$ be a vertex in $\delta_{k}$ for some $0 \leq k \leq n$. Then there exists a 1 -skeleton geodesic $\left(v_{i}\right)_{i=0}^{n}$ such that $v_{i} \in \delta_{i}$ for $0 \leq i \leq n$. This follows from the fact that $\delta_{i+1} \subset S_{1}\left(\delta_{i}\right)$, which we use for $1 \leq i<k$, and from $\delta_{i} \subset S_{1}\left(\delta_{i+1}\right)$, which we use for $k \leq i<n-1$ (see Section 1 or Lemma 9.15(i)).

Definition 3.2 Let $v, w$ be vertices of a systolic complex $X$. Let $\gamma=\left(v_{0}=\right.$ $\left.v, v_{1}, v_{2}, \ldots, v_{n}=w\right)$ be a geodesic in the 1-skeleton of $X$ between $v$ and $w$ or let $\gamma=\left(v=v_{0}, v_{1}, v_{2}, \ldots\right)$ be a 1 -skeleton geodesic ray starting at $v$ (then we set $n=\infty)$. For $0 \leq i<j \leq n$, we denote the Euclidean geodesic between $v_{i}$ and $v_{j}$ by $\left(\delta_{i}^{i, j}=v_{i}, \delta_{i+1}^{i, j}, \ldots, \delta_{j}^{i, j}=v_{j}\right)$. We say that $\gamma$ is a good geodesic between $v$ and $w$ or that $\gamma$ is a good geodesic ray starting at $v$ if for every $0 \leq i<j \leq n$ and every $i \leq k \leq j$ we have $\left|v_{k}, \delta_{k}^{i, j}\right| \leq C+1$ (the constant $C$ is defined at the beginning of this section).

We denote the set of all good geodesic rays in $X$ by $\mathcal{R}$. For a given vertex $O$ of $X$, we denote the set of all good geodesic rays starting at $O$ by $\mathcal{R}_{O}$.

The following two results are immediate corollaries of Theorem B and Theorem C.

Corollary 3.3 For every two vertices $v, w \in X$ there exists a good geodesic between them.

Proof Let $\left(\delta_{0}=v, \delta_{1}, \ldots, \delta_{n}=w\right)$ be the Euclidean geodesic between $v$ and $w$. By Remark 3.1, there exists a 1 -skeleton geodesic $\gamma=\left(v_{0}=v, v_{1}, v_{2}, \ldots, v_{n}=w\right)$ with $v_{i} \in \delta_{i}$. We claim that $\gamma$ is a good geodesic. To justify the claim let $0 \leq i<j \leq n$. Let $\left(\tilde{\delta}_{i}, \tilde{\delta}_{i+1}, \ldots, \tilde{\delta}_{j}\right)$ be the Euclidean geodesic between $v_{i}$ and $v_{j}$. By Theorem B, for every $i \leq k \leq j$, we have

$$
\left|v_{k}, \tilde{\delta}_{k}\right| \leq\left|\delta_{k}, \tilde{\delta}_{k}\right|+1 \leq C+1
$$

which justifies the claim.

Corollary 3.4 Let $\left(v_{0}=O, v_{1}, v_{2}, \ldots, v_{n}\right),\left(w_{0}=O, w_{1}, w_{2}, \ldots, w_{m}\right)$ be good geodesics in $X$. Then for all $0 \leq c \leq 1$ we have $\left|v_{\lfloor c n\rfloor} w_{\lfloor c m\rfloor}\right| \leq c\left|v_{n} w_{m}\right|+D$, where $D=3 C+2$.

Proof Let $\left(\delta_{i}^{v}\right),\left(\delta_{i}^{w}\right)$ be the Euclidean geodesics between $O$ and $v_{n}, w_{m}$, respectively. Fix $0 \leq c \leq 1$. Pick vertices $v_{\lfloor c n\rfloor}^{\prime} \in \delta_{\lfloor c n\rfloor}^{v}$ and $w_{\lfloor c m\rfloor}^{\prime} \in \delta_{\lfloor c m\rfloor}^{w}$ which realize the distance to $v_{\lfloor c n\rfloor}, w_{\lfloor c m\rfloor}$, respectively. Find 1 -skeleton geodesics $\left(v_{i}^{\prime}\right)_{i=0}^{n}$ and $\left(w_{i}^{\prime}\right)_{i=0}^{m}$ such 
that $v_{i}^{\prime} \in \delta_{i}^{v}$ and $w_{i}^{\prime} \in \delta_{i}^{w}$. Their existence is guaranteed by Remark 3.1. By Theorem C, we have

$$
\begin{aligned}
\left|v_{\lfloor c n\rfloor} w_{\lfloor c m\rfloor}\right| & \leq\left|v_{\lfloor c n\rfloor} v_{\lfloor c n\rfloor}^{\prime}\right|+\left|v_{\lfloor c n\rfloor}^{\prime} w_{\lfloor c m\rfloor}^{\prime}\right|+\left|w_{\lfloor c m\rfloor}^{\prime} w_{\lfloor c m\rfloor}\right| \\
& =\left|v_{\lfloor c n\rfloor}, \delta_{\lfloor c n\rfloor}^{v}\right|+\left|v_{\lfloor c n\rfloor}^{\prime} w_{\lfloor c m\rfloor}^{\prime}\right|+\left|\delta_{\lfloor c m\rfloor}^{w}, w_{\lfloor c m\rfloor}\right| \\
& \leq(C+1)+\left(c\left|v_{n} w_{m}\right|+C\right)+(C+1),
\end{aligned}
$$

as desired.

The following simple corollary of Corollary 3.4 will be useful.

Corollary 3.5 Let $\left(v_{0}=O, v_{1}, v_{2}, \ldots, v_{k}\right),\left(w_{0}=O, w_{1}, w_{2}, \ldots, w_{l}\right)$ be good geodesics in $X$. Then for all $0 \leq N \leq \min \{k, l\}$ we have $\left|v_{N} w_{N}\right| \leq 2\left|v_{k} w_{l}\right|+D$.

Proof Without loss of generality we can assume that $k \leq l$. Observe that $l-k \leq\left|v_{k} w_{l}\right|$. Hence, by Corollary 3.4, we have

$$
\begin{aligned}
\left|v_{N} w_{N}\right| \leq\left|v_{k} w_{k}\right|+D & \leq\left|v_{k} w_{l}\right|+\left|w_{l} w_{k}\right|+D \\
& =\left|v_{k} w_{l}\right|+(l-k)+D \leq 2\left|v_{k} w_{l}\right|+D .
\end{aligned}
$$

Below we define the central object of the article.

Definition 3.6 The ideal boundary (or shortly the boundary) of a systolic complex $X$ is the set $\partial X=\mathcal{R} / \sim$ of equivalence classes of good geodesic rays, where rays $\eta=\left(v_{0}, v_{1}, v_{2}, \ldots\right), \xi=\left(w_{0}, w_{1}, w_{2}, \ldots\right)$ are identified if $\left|v_{i} w_{i}\right|$ is bounded above by a constant independent of $i$ (one can check that this happens exactly when the Hausdorff distance between $\eta$ and $\xi$ is finite). For a good geodesic ray $\eta$, we denote its equivalence class in $\partial X$ by $[\eta]$.

In order to introduce topology on $\bar{X}=X \cup \partial X$ we give another definition of the boundary. The two definitions will turn out to be equivalent in the case of a systolic complex with a geometric group action.

Definition 3.7 Let $O$ be a vertex of a systolic complex $X$. Then the (ideal) boundary of $X$ with respect to the basepoint vertex $O$ is the set $\partial_{O} X=\mathcal{R}_{O} / \sim$ of equivalence classes of good geodesic rays starting at $O$, where rays $\eta=\left(v_{0}=O, v_{1}, v_{2}, \ldots\right), \xi=$ $\left(w_{0}=O, w_{1}, w_{2}, \ldots\right)$ are identified if $\left|v_{i} w_{i}\right|$ is bounded above by a constant independent of $i$ (again this happens exactly when the Hausdorff distance between $\eta$ and $\xi$ is finite). For $\eta \in \mathcal{R}_{O}$, we denote its equivalence class in $\partial_{O} X$ by $[\eta]$ (we hope this ambiguity of the notation will not cause confusion). 
Lemma 3.8 Let $\eta=\left(v_{0}=O, v_{1}, v_{2}, \ldots\right), \xi=\left(w_{0}=O, w_{1}, w_{2}, \ldots\right) \in \mathcal{R}_{O}$. Then $[\eta]=[\xi]$ if and only if $\left|v_{i} w_{i}\right| \leq D$ for all $i$.

Proof We show that if for some $i$ we have $\left|v_{i} w_{i}\right|-D \geq 1$, then $[\xi] \neq[\eta]$. Let $i$ be as above and $R$ be a natural number. Then, by Corollary 3.4, we have

$$
\left|v_{R i} w_{R i}\right| \geq R\left(\left|v_{i} w_{i}\right|-D\right) \geq R .
$$

Since $R$ can be chosen arbitrarily large, we get $[\xi] \neq[\eta]$.

In the remaining part of this section we prove equivalence of the above two notions of boundaries in the case of locally finite complexes. Assume that $X$ is a locally finite systolic complex. Let $O \in X$ be a fixed vertex and let $\eta=\left(v^{0}, v^{1}, v^{2}, \ldots\right)$ be a good geodesic ray in $X$. For every $i \geq 0$ we choose a good geodesic $\eta^{i}=$ $\left(v_{0}^{i}=O, v_{1}^{i}, v_{2}^{i}, \ldots, v_{n(i)}^{i}=v^{i}\right)$, guaranteed by Corollary 3.3. Since $B_{1}(O)$ is finite, for some vertex $v_{1} \in S_{1}(O)$ there are infinitely many $i$ such that $n(i)=\left|O v^{i}\right| \geq 1$ and $v_{1}^{i}=v_{1}$. Similarly, since all balls are finite, we obtain inductively vertices $v_{k} \in S_{k}(O)$ satisfying the following. For each $k$ there are infinitely many $i$ such that $n(i) \geq k$ and for all $j \leq k$ we have $v_{j}^{i}=v_{j}$. For each $k$ denote some such $i$ by $i(k)$. The following easy facts hold.

Lemma 3.9 The sequence $\left(v_{0}=O, v_{1}, v_{2}, \ldots\right)$ obtained as above is a good geodesic ray. Moreover, for every $j$ we have $\left|v^{j} v_{j}\right| \leq 3\left|O v^{0}\right|+D$.

Proof The first assertion follows from the fact that for every $k$ the sequence $\left(v_{0}=O\right.$, $\left.v_{1}, v_{2}, \ldots, v_{k}\right)$ is a subsequence of the good geodesic $\eta^{i(k)}$ and hence, by Definition 3.2 , it is a good geodesic.

Now we prove the second assertion. Let $j \geq 0$. Consider the case of $n(i(j)) \leq i(j)$ (the case of $n(i(j))>i(j)$ can be examined analogously). Then for $k=i(j)-n(i(j))$ we have $\left|v^{k} v^{i(j)}\right|=\left|v_{0}^{i(j)} v^{i(j)}\right|$. Thus we can apply Corollary 3.4 with $m=n$ to good geodesics $\eta^{i(j)}$ and $\left(v^{k}, v^{k+1}, \ldots, v^{i(j)}\right)$, which yields the following.

$$
\left|v^{k+j} v_{j}\right|=\left|v^{k+j} v_{j}^{i(j)}\right| \leq\left|v^{k} v_{0}^{i(j)}\right|+D \leq\left(\left|O v^{0}\right|+k\right)+D .
$$

Hence

$$
\left|v^{j} v_{j}\right| \leq k+\left|v^{k+j} v_{j}\right| \leq\left|O v^{0}\right|+2 k+D \leq 3\left|O v^{0}\right|+D,
$$

where the last inequality follows from $k \leq\left|O v^{0}\right|$, which is the triangle inequality for $v^{0}, v^{i(j)}$ and $O$.

Corollary 3.10 Let $X$ be a locally finite systolic complex and $O, O^{\prime}$ its vertices. Then the map $\Phi_{O}: \partial X \rightarrow \partial_{O} X$ given by $\Phi_{O}\left(\left[\left(v^{0}, v^{1}, v^{2}, \ldots\right)\right]\right)=\left[\left(v_{0}=O, v_{1}, v_{2}, \ldots\right)\right]$ is well defined. It is a bijection between $\partial X$ and $\partial_{O} X$. Its restriction $\Phi_{O^{\prime} O}=\left.\Phi_{O}\right|_{\partial_{O^{\prime}} X}$ is a bijection between $\partial_{O^{\prime}} X$ and $\partial_{O} X$. 


\section{Topology on $\bar{X}=X \cup \partial_{o} X$}

Let $X$ be a systolic complex and $O \in X$ be its vertex. In this section we define the topology on the set $\bar{X}=X \cup \partial_{O} X$, which extends the usual topology on the simplicial complex $X$. The idea is to define the topology through neighborhoods (not necessarily open) of points in $\bar{X}$. The only problem is to define the neighborhoods of points in the boundary.

For a 1 -skeleton geodesic or geodesic ray $\eta=\left(v_{0}, v_{1}, v_{2}, \ldots\right)$, we denote by $B_{1}(\eta)$ the combinatorial ball of radius 1 around the subcomplex $\left\{v_{0}, v_{1}, v_{2}, \ldots\right\}$. Let $C$ and $D=3 C+2$ be the constants from the previous section.

Definition 4.1 Let $\eta=\left(v_{0}=O, v_{1}, v_{2}, \ldots\right)$ be a good geodesic ray in $X$ and let $R>D$ (ie $R \geq D+1$ ) and $N \geq 1$ be natural numbers (in fact we could also allow $N=0$, but this would complicate some computations later on). By $\mathcal{G}_{O}(\eta, N, R)$ we denote the set of all good geodesics $\left(w_{0}=O, w_{1}, \ldots, w_{k}\right)$ with $k \geq N$ and good geodesic rays $\left(O=w_{0}, w_{1}, \ldots\right)$, such that $\left|w_{N} v_{N}\right| \leq R$. By $\mathcal{G}^{\prime} O(\eta, N, R)$ we denote the set

$$
\left\{\left(w_{N}, w_{N+1}, \ldots\right) \mid\left(w_{0}=O, w_{1}, \ldots\right) \in \mathcal{G}_{O}(\eta, N, R)\right\} .
$$

A standard neighborhood of $[\eta] \in \partial_{O} X \subset \bar{X}$ is the set

$$
U_{O}(\eta, N, R)=\left\{[\xi] \mid \xi \in \mathcal{G}_{O}(\eta, N, R) \cap \mathcal{R}_{O}\right\} \cup \bigcup\left\{\operatorname{int} B_{1}(\xi) \mid \xi \in \mathcal{G}^{\prime}{ }_{O}(\eta, N, R)\right\} .
$$

If it is clear what is the basepoint $O$, we write $\mathcal{G}(\eta, N, R), \mathcal{G}^{\prime}(\eta, N, R)$ and $U(\eta, N, R)$ instead of $\mathcal{G}_{O}(\eta, N, R), \mathcal{G}^{\prime} O(\eta, N, R)$ and $U_{O}(\eta, N, R)$.

Before we define the topology, we need the following useful lemmas. The first one is an immediate consequence of the above definition.

Lemma 4.2 Let $\eta, \xi \in \mathcal{R}_{O}$ and let $N, N^{\prime}, R>D, R^{\prime}>D$ be natural numbers such that $N^{\prime} \geq N$. If $\mathcal{G}\left(\xi, N^{\prime}, R^{\prime}\right) \subset \mathcal{G}(\eta, N, R)$ then $U\left(\xi, N^{\prime}, R^{\prime}\right) \subset U(\eta, N, R)$.

Lemma 4.3 Let $U(\eta, N, R)$ be a standard neighborhood, let $\xi \in \mathcal{R}_{O}$ be such that $[\xi]=[\eta]$ and let $R^{\prime}>D$ be a natural number. Then, for $N^{\prime} \geq\left(R^{\prime}+D\right) N$, we have $U\left(\xi, N^{\prime}, R^{\prime}\right) \subset U(\eta, N, R)$.

Proof Denote $\eta=\left(v_{0}=O, v_{1}, v_{2}, \ldots\right)$ and $\xi=\left(w_{0}=O, w_{1}, w_{2}, \ldots\right)$.

By Lemma 4.2, it is enough to show that for every $\zeta \in \mathcal{G}\left(\xi, N^{\prime}, R^{\prime}\right)$ we have $\zeta \in$ $\mathcal{G}(\eta, N, R)$. 
Let $\zeta=\left(z_{0}=O, z_{1}, z_{2}, \ldots\right) \in \mathcal{G}\left(\xi, N^{\prime}, R^{\prime}\right)$. By Corollary 3.4 and Lemma 3.8, we have

$$
\begin{aligned}
\left|z_{N} v_{N}\right| & \leq \frac{1}{R^{\prime}+D}\left|z_{N^{\prime}} v_{N^{\prime}}\right|+D \leq \frac{1}{R^{\prime}+D}\left(\left|z_{N^{\prime}} w_{N^{\prime}}\right|+\left|w_{N^{\prime}} v_{N^{\prime}}\right|\right)+D \\
& \leq \frac{1}{R^{\prime}+D}\left(R^{\prime}+D\right)+D \leq R .
\end{aligned}
$$

Thus $\zeta \in \mathcal{G}(\eta, N, R)$ and the lemma follows.

The following defines topology on $\bar{X}$.

Proposition 4.4 Let $\mathcal{A}$ be the family of subsets $A$ of $\bar{X}=X \cup \partial_{O} X$ satisfying the following. $A \cap X$ is open in $X$ and for every $x \in A \cap \partial_{O} X$ there is some $\eta \in \mathcal{R}_{O}$ such that $[\eta]=x$ and there is a standard neighborhood $U(\eta, N, R) \subset A$. Then $\mathcal{A}$ is a topology on $\bar{X}$.

Proof The only thing we have to check is the following. If $A_{1}, A_{2} \in \mathcal{A}$ and $[\eta] \in$ $A_{1} \cap A_{2} \cap \partial_{O} X$, then there is a standard neighborhood $U(\eta, N, R)$ of $[\eta]$ contained in $A_{1} \cap A_{2}$.

Since $A_{i} \in \mathcal{A}$, for $i=1,2$, there are standard neighborhoods $U\left(\eta_{i}, N_{i}, R_{i}\right) \subset A_{i}$ such that $\left[\eta_{i}\right]=[\eta]$. Thus, by Lemma 4.3, for any natural $R>D$ there exists $N \geq N_{i}$, $i=1,2$, such that $U(\eta, N, R) \subset U\left(\eta_{1}, N_{1}, R_{1}\right) \cap U\left(\eta_{2}, N_{2}, R_{2}\right) \subset A_{1} \cap A_{2}$.

Remark 4.5 The boundary $\partial_{O} X$ is a closed subset of $\bar{X}=X \cup \partial_{O} X$.

Remark 4.6 It is easy to verify that when $X$ is $\delta$-hyperbolic (in the sense of Gromov) then our boundary $\partial_{O} X$ (with topology induced from $\bar{X}$ ) is homeomorphic in a natural way with the Gromov boundary of $X$.

We still did not prove that the topology defined in Proposition 4.4 is nontrivial. This will follow from the next two lemmas, in which we characterize the intersections with the boundary of the interiors of standard neighborhoods. In particular, we show (in Lemma 4.8) that $[\xi]$ is contained in the interior of $U(\xi, N, R)$.

Lemma 4.7 For a set $A \subset \bar{X}$, the intersection int $A \cap \partial_{O} X$ consists of those points $x \in \partial_{O} X$ for which there exists a representative $\eta$ with a standard neighborhood $U(\eta, N, R) \subset A$. 
Proof Let $B$ be the set of those points $x \in \partial_{O} X$ for which there exists a representative $\eta$ of $x$ with a standard neighborhood $U(\eta, N, R) \subset A$.

It is clear that int $A \cap \partial_{O} X \subset B$, since int $A$ is open in the topology defined in Proposition 4.4. We want now to prove the converse inclusion $B \subset$ int $A \cap \partial_{O} X$. It is clear that $B \subset A \cap \partial_{O} X$. Thus to prove the lemma we only have to show that $B$ is open in $\partial_{O} X$ (in the topology induced from $\bar{X}$ ).

Let $x \in B$ and let its representative $\eta$ be such that the standard neighborhood $U\left(\eta, N, R^{\prime}\right)$ is contained in $A$. By Lemma 4.3, we can assume that $R^{\prime} \geq 2(D+1)$. Choose natural numbers $R>D$ and $N^{\prime} \geq R N$. We claim that $U\left(\eta, N^{\prime}, R\right) \cap \partial_{O} X \subset B$ (ie that equivalence classes of elements in $\mathcal{G}\left(\eta, N^{\prime}, R\right) \cap \mathcal{R}_{O}$ lie in $B$ ). This implies that $B$ is open in $\partial_{O} X$.

To justify the claim let $\xi \in \mathcal{G}\left(\eta, N^{\prime}, R\right) \cap \mathcal{R}_{O}$. To prove that $[\xi] \in B$ it is enough to establish $U\left(\xi, N^{\prime}, R\right) \subset U\left(\eta, N, R^{\prime}\right)$, since the latter is contained in $A$. By Lemma 4.2 , it is enough to show that for every $\zeta \in \mathcal{G}\left(\xi, N^{\prime}, R\right)$, we have $\zeta \in \mathcal{G}\left(\eta, N, R^{\prime}\right)$. Let $\zeta=\left(z_{0}=O, z_{1}, \ldots\right) \in \mathcal{G}\left(\xi, N^{\prime}, R\right)$. Denote $\eta=\left(v_{0}=O, v_{1}, \ldots\right), \xi=\left(w_{0}=\right.$ $\left.O, w_{1}, \ldots\right)$.

By Corollary 3.4, we have

$$
\begin{aligned}
\left|z_{N} v_{N}\right| & \leq\left|z_{N} w_{N}\right|+\left|w_{N} v_{N}\right| \\
& \leq\left(\frac{1}{R}\left|z_{N^{\prime}} w_{N^{\prime}}\right|+D\right)+\left(\frac{1}{R}\left|w_{N^{\prime}} v_{N^{\prime}}\right|+D\right) \\
& \leq\left(\frac{1}{R} R+D\right)+\left(\frac{1}{R} R+D\right)=2(D+1) \leq R^{\prime} .
\end{aligned}
$$

Thus $\zeta \in \mathcal{G}\left(\eta, N, R^{\prime}\right)$ and it follows that $U\left(\xi, N^{\prime}, R\right) \subset U\left(\eta, N, R^{\prime}\right)$, which justifies the claim.

Lemma 4.8 Suppose that $U(\eta, N, R)$ is a standard neighborhood and suppose $\xi=$ $\left(w_{0}=O, w_{1}, w_{2}, \ldots\right) \in \mathcal{R}_{O}$ is such that $v_{N}=w_{N}$, where $\eta=\left(v_{0}=O, v_{1}, v_{2}, \ldots\right)$. Then $[\xi]$ is contained in the interior of $U(\eta, N, R)$.

Proof By Lemma 4.7, it is enough to show that there exists a standard neighborhood $U\left(\xi, N^{\prime}, R\right)$ of $[\xi]$ contained in $U(\eta, N, R)$. Let $N^{\prime} \geq R N$. By Lemma 4.2, it is enough to show that for $\left(z_{0}=O, z_{1}, z_{2}, \ldots\right) \in \mathcal{G}\left(\xi, N^{\prime}, R\right)$, we have $\left|z_{N} v_{N}\right| \leq R$. By Corollary 3.4 , we have

$$
\left|z_{N} v_{N}\right|=\left|z_{N} w_{N}\right| \leq \frac{1}{R}\left|z_{N^{\prime}} w_{N^{\prime}}\right|+D \leq \frac{1}{R} R+D \leq R,
$$

as desired. 
Below we give a sufficient condition for two standard neighborhoods to be disjoint.

Lemma 4.9 Let $U(\eta, N, R)$ and $U(\xi, N, S)$ be two standard neighborhoods, with $\eta=\left(v_{0}=O, v_{1}, v_{2}, \ldots\right)$ and $\xi=\left(w_{0}=O, w_{1}, w_{2}, \ldots\right)$. If $\left|v_{N} w_{N}\right|>R+S+D+2$, then $U(\eta, N, R) \cap U(\xi, N, S)=\varnothing$.

Proof By contradiction. Assume $U(\eta, N, R) \cap U(\xi, N, S) \neq \varnothing$.

Case 1 Let $x \in U(\eta, N, R) \cap U(\xi, N, S) \cap X$. Then, by Definition 4.1, there exist $\eta^{\prime}=\left(v_{0}^{\prime}=O, v_{1}^{\prime}, v_{2}^{\prime}, \ldots\right) \in \mathcal{G}(\eta, N, R)$ and $\xi^{\prime}=\left(w_{0}^{\prime}=O, w_{1}^{\prime}, w_{2}^{\prime}, \ldots\right) \in \mathcal{G}(\xi, N, S)$ such that $x$ belongs to the interior of both some simplex with vertex $v_{k}^{\prime}$ and some simplex with vertex $w_{l}^{\prime}$, for some $k, l \geq N$. Then these simplices coincide and $\left|v_{k}^{\prime} w_{l}^{\prime}\right| \leq 1$. By Corollary 3.5, we have

$\left|v_{N} w_{N}\right| \leq\left|v_{N} v_{N}^{\prime}\right|+\left|v_{N}^{\prime} w_{N}^{\prime}\right|+\left|w_{N}^{\prime} w_{N}\right| \leq R+\left(2\left|v_{k}^{\prime} w_{l}^{\prime}\right|+D\right)+S \leq R+(2+D)+S$, which is a contradiction.

Case 2 Let $\eta^{\prime}=\left(v_{0}^{\prime}=O, v_{1}^{\prime}, v_{2}^{\prime}, \ldots\right) \in \mathcal{G}(\eta, N, R)$ and $\xi^{\prime}=\left(w_{0}^{\prime}=O, w_{1}^{\prime}, w_{2}^{\prime}, \ldots\right) \in$ $\mathcal{G}(\xi, N, S)$ be such that $\left[\eta^{\prime}\right]=\left[\xi^{\prime}\right]$. Then, by Lemma 3.8, we get

$$
\left|v_{N} w_{N}\right| \leq\left|v_{N} v_{N}^{\prime}\right|+\left|v_{N}^{\prime} w_{N}^{\prime}\right|+\left|w_{N}^{\prime} w_{N}\right| \leq R+D+S,
$$

which is a contradiction.

\section{Compactness and finite dimensionality}

Let $X$ be a locally finite systolic complex and let $O \in X$ be its vertex. In this section we show that $\bar{X}=X \cup \partial_{O} X$ is compact metrizable and (if $X$ satisfies some additional local finiteness conditions) finitely dimensional. We also prove that, for a different vertex $O^{\prime}$ of $X$, the compactifications $X \cup \partial_{O} X$ and $X \cup \partial_{O^{\prime}} X$ are homeomorphic.

Proposition 5.1 If $X$ is locally finite then the space $\bar{X}=X \cup \partial_{O} X$ is second countable and regular.

Proof It is clear that $\bar{X}$ is second countable. We show that $\bar{X}$ is regular.

First we show that $\bar{X}$ is Hausdorff. We consider only the case of two points of the boundary-the other cases are obvious. Let $[\eta] \neq[\xi]$ be two boundary points with $\eta=\left(v_{0}=O, v_{1}, v_{2}, \ldots\right)$ and $\xi=\left(w_{0}=O, w_{1}, w_{2}, \ldots\right)$. Fix a natural number $R>D$ (for example $R=D+1$ ). We can find $N$ such that $\left|v_{N} w_{N}\right|>2 R+D+2$. Then, by Lemma 4.8, we have $[\eta] \in \operatorname{int} U(\eta, N, R)$ and $[\xi] \in \operatorname{int} U(\xi, N, R)$ and, by Lemma 4.9, 
we get int $U(\eta, N, R) \cap \operatorname{int} U(\xi, N, R) \subset U(\eta, N, R) \cap U(\xi, N, R)=\varnothing$. Thus we get disjoint nonempty open neighborhoods of $[\eta]$ and $[\xi]$.

To show that $\bar{X}$ is regular it now suffices to find, for every point $x \in \bar{X}$ and every closed subset $A \subset \bar{X}$ which does not contain $x$, disjoint open sets $U, V$ such that $x \in U$ and $A \subset V$. The case of $x \in X$ is obvious, hence we consider only the case of $x=[\eta] \in \partial_{O} X$, for $\eta=\left(v_{0}=O, v_{1}, v_{2}, \ldots\right)$. Fix some natural $R>D$. Since $\bar{X} \backslash A$ is open, by the definition of the topology (Proposition 4.4) and by Lemma 4.3 , we can find a natural number $N>0$ such that $U\left(\eta, N, R^{\prime}\right) \subset \bar{X} \backslash A$, where $R^{\prime} \geq 2 D+2$. Let $N^{\prime}=(R+1) N+1$ and let $U=\operatorname{int} U\left(\eta, N^{\prime}, R\right)$. Observe that, by Lemma 4.8, we have $x \in U$. Now we define $V$. For each $y \in A \cap X$, choose an open set $V_{y}=\operatorname{int} B_{1}\left(z^{\prime}\right)$ for some vertex $z^{\prime}$ in $X$ such that $y \in \operatorname{int} B_{1}\left(z^{\prime}\right)$. Then we set $V=\bigcup\left\{V_{y} \mid y \in A \cap X\right\} \cup \bigcup\left\{\operatorname{int} U\left(\xi, N^{\prime}, R\right) \mid[\xi] \in A \cap \partial_{O} X\right\}$. By Lemma 4.8, we have $A \cap \partial_{O} X \subset V$, hence $A \subset V$. Thus to prove that $U$ and $V$ are as desired we only need to show that $U \cap V=\varnothing$.

First we prove that $U \cap \operatorname{int} U\left(\xi, N^{\prime}, R\right)=\varnothing$, for $[\xi] \in A \cap \partial_{O} X$. Let $\xi=$ $\left(w_{0}=O, w_{1}, w_{2}, \ldots\right)$. Then, by Corollary 3.4 and by $A \cap U\left(\eta, N, R^{\prime}\right)=\varnothing$, we have

$$
\begin{aligned}
\left|v_{N^{\prime}} w_{N^{\prime}}\right| & \geq \frac{N^{\prime}}{N}\left(\left|v_{N} w_{N}\right|-D\right)>(R+1)\left(R^{\prime}-D\right) \\
& \geq(R+1)(D+2)>2 R+D+2 .
\end{aligned}
$$

Thus, by Lemma 4.9, $U \cap \operatorname{int} U\left(\xi, N^{\prime}, R\right) \subset U\left(\eta, N^{\prime}, R\right) \cap U\left(\xi, N^{\prime}, R\right)=\varnothing$.

Now we show that $U \cap V_{y}=\varnothing$, for $y \in A \cap X$. By contradiction, assume $p \in U \cap V_{y}$. Since $p \in U$, there exist a vertex $z$ of the simplex containing $p$ in its interior and a good geodesic $\left(z_{0}=O, z_{1}, \ldots, z_{k}=z\right) \in \mathcal{G}\left(\eta, N^{\prime}, R\right)$, where $k \geq N^{\prime}$. Then, by Corollary 3.4 , we have

$$
\left|v_{N} z_{N}\right| \leq \frac{N}{N^{\prime}}\left|v_{N^{\prime}} z_{N^{\prime}}\right|+D<\frac{1}{R} R+D \leq D+1 .
$$

On the other hand, since $p \in V_{y}$, there is a vertex $z^{\prime}$ such that $\{y, p\} \in \operatorname{int} B_{1}\left(z^{\prime}\right)$. Then $\left|z z^{\prime}\right| \leq 1$. Let $\left(O=z_{0}^{\prime}, z_{1}^{\prime}, \ldots, z_{l}^{\prime}=z^{\prime}\right)$ be a good geodesic. We have $l \geq N^{\prime}-1$, hence by Corollary 3.4 and Corollary 3.5, we get

$$
\begin{aligned}
\left|z_{N} z_{N}^{\prime}\right| & \leq \frac{N}{N^{\prime}-1}\left|z_{N^{\prime}-1} z_{N^{\prime}-1}^{\prime}\right|+D \leq \frac{1}{R+1}\left(2\left|z z^{\prime}\right|+D\right)+D \\
& \leq \frac{1}{D+2}(2+D)+D=D+1 .
\end{aligned}
$$

Summarizing, we have $\left|v_{N} z_{N}^{\prime}\right| \leq\left|v_{N} z_{N}\right|+\left|z_{N} z_{N}^{\prime}\right| \leq 2 D+2 \leq R^{\prime}$. It follows that $\left(O=z_{0}^{\prime}, z_{1}^{\prime}, \ldots, z_{L}^{\prime}=z^{\prime}\right) \in \mathcal{G}\left(\eta, N, R^{\prime}\right)$ and hence $y \in U\left(\eta, N, R^{\prime}\right)$-contradiction. 
Corollary 5.2 If $X$ is locally finite then the space $\bar{X}=X \cup \partial_{O} X$ is metrizable.

Proof This follows from the Urysohn Metrization Theorem—cf Dugundji [13, Corollary 9.2].

Proposition 5.3 If $X$ is locally finite then the space $\bar{X}=X \cup \partial_{O} X$ is compact.

Proof By Corollary 5.2, it is enough to show that every infinite sequence of points in $\bar{X}$ contains a convergent subsequence. Let $\left(x^{1}, x^{2}, x^{3}, \ldots\right)$ be a given sequence of points in $\bar{X}$. If for some $n>0$ there are only finitely many $x^{i}$ outside the ball $B_{n}(O)$ (which is finite), then we can find a convergent subsequence. From now on we assume there is no $n$ as above.

For every $i$ we choose a good geodesic or a good geodesic ray $\eta^{i}=\left(v_{0}^{i}=O, v_{1}^{i}, v_{2}^{i}, \ldots\right)$ in the following way. If $x^{i} \in X$ then $\eta^{i}=\left(v_{0}^{i}=O, v_{1}^{i}, v_{2}^{i}, \ldots, v_{n(i)}^{i}\right)$ is a good geodesic between $O$ and a vertex $v_{n(i)}^{i}$ lying in a common simplex with the point $x^{i}$. If $x^{i} \in \partial_{O} X$ then we take $\eta^{i}$ so that $x^{i}=\left[\eta^{i}\right]$ and we set $n(i)=\infty$. By our assumptions on $\left(x^{1}, x^{2}, x^{3}, \ldots\right)$, for every $n>0$ there exists an arbitrarily large $i$ such that $n(i)>n$. Since $S_{1}(O)$ is finite, for some vertex $v_{1} \in S_{1}(O)$ there are infinitely many $i$ such that $n(i) \geq 1$ and $v_{1}^{i}=v_{1}$. Let $i(1)$ be some such $i$. Similarly, since all spheres are finite, we obtain inductively vertices $v_{k} \in S_{k}(O)$ and numbers $i(k)$ satisfying the following. For each $k$ there are infinitely many $i$ such that $n(i) \geq k$ and for all $j \leq k$ we have $v_{j}^{i}=v_{j}$; we denote some such $i>i(k-1)$ by $i(k)$.

Observe that for every $k$ the sequence $\left(v_{0}=O, v_{1}, v_{2}, \ldots, v_{k}\right)$ is a subsequence of the good geodesic or the good geodesic ray $\eta^{i(k)}$ and hence, by Definition 3.2, it is a good geodesic. Thus every subsequence of the infinite sequence $\left(v_{0}=O, v_{1}, v_{2}, \ldots\right)$ is a good geodesic and again, by Definition 3.2, $\left(v_{0}=O, v_{1}, v_{2}, \ldots\right)$ is a good geodesic ray.

We claim that the sequence $\left(x^{i(k)}\right)_{k=1}^{\infty}$ of points of $\bar{X}$ converges to $[\eta] \in \partial_{O} X$, where $\eta=\left(v_{0}=O, v_{1}, v_{2}, \ldots\right)$. To prove the claim it is enough to show (since every open set containing $[\eta]$ contains some $U(\eta, N, R)$, by Lemma 4.3) that we have $\eta^{i(k)} \in \mathcal{G}(\eta, N, R)$, for every $k \geq N$. This follows from the equality $v_{N}^{i(k)}=v_{N}$, which holds for every $k \geq N$.

Observe that by the above proof we get the following.

Corollary 5.4 If a locally finite systolic complex is unbounded then its boundary is nonempty. 
Below we prove that the bijection $\Phi_{O^{\prime} O}$ defined in Corollary 3.10 extends to a homeomorphism of compactifications coming from different basepoints.

Lemma 5.5 Let $X$ be a locally finite systolic complex and let $O, O^{\prime}$ be its vertices. Then the map $\Phi_{O^{\prime} O}: X \cup \partial_{O^{\prime}} X \rightarrow X \cup \partial_{O} X$ defined as an extension by identity on $X$ of the map $\Phi_{O^{\prime} O}: \partial_{O^{\prime}} X \rightarrow \partial_{O} X$ is a homeomorphism.

Proof By compactness (Proposition 5.3) and by Corollary 3.10, we only have to show that $\Phi_{O^{\prime} O}$ is continuous. It is enough to check the continuity at the boundary points. Let $\xi=\left(v_{0}=O, v_{1}, v_{2}, \ldots\right)$ be obtained from a good geodesic ray $\eta=$ $\left(v^{0}=O^{\prime}, v^{1}, v^{2}, \ldots\right)$ as in the definition of the map $\Phi_{O^{\prime} O}$. We show that $\Phi_{O^{\prime} O}$ is continuous at [ $\eta$ ]. Let $d=\left|O O^{\prime}\right|$, let $R>D$ be a natural number, let $R^{\prime}=R+3 D+6 d$ and let $U$ be an open neighborhood of $[\xi]$ in $X \cup \partial_{O} X$. We have to show that there exists an open neighborhood $V$ of $[\eta]$ in $X \cup \partial_{O^{\prime}} X$ such that $\Phi_{O^{\prime}} O(V) \subset U$. By Lemma 4.3, there exists $N$ such that $U_{O}\left(\xi, N, R^{\prime}\right) \subset U$. Let $V=\operatorname{int} U_{O^{\prime}}(\eta, N+d, R)$. By Lemma 4.8, $[\eta] \in V$. We claim that $\Phi_{O^{\prime}} O(V) \subset U$-this will finish the proof.

First we show that for $x \in V \cap X$ we have $\Phi_{O^{\prime} O}(x)=x \in U$. For such an $x$ choose, by the definition of $U_{O^{\prime}}(\eta, N+d, R)$, a good geodesic $\left(w^{0}=O^{\prime}, w^{1}, w^{2}, \ldots, w^{k}\right) \in$ $\mathcal{G}_{O^{\prime}}(\eta, N+d, R)$ such that $x$ belongs to the interior of a simplex with vertex $w^{k}$, where $k \geq N+d$. Let $\zeta=\left(w_{0}=O, w_{1}, w_{2}, \ldots, w_{l}=w^{k}\right)$ be a good geodesic guaranteed by Corollary 3.3. Then $|l-k| \leq d$, hence $l \geq N$ and $w_{N}$ is defined. By Lemma 3.9 and Corollary 3.4, we have

$$
\begin{aligned}
\left|w_{N} v_{N}\right| & \leq\left|w_{N} w^{N}\right|+\left|w^{N} v^{N}\right|+\left|v^{N} v_{N}\right| \\
& \leq(3 d+D)+\left(\left|w^{N+d} v^{N+d}\right|+D\right)+(3 d+D) \\
& \leq R+3 D+6 d=R^{\prime} .
\end{aligned}
$$

This inequality implies that $\zeta \in \mathcal{G}_{O}\left(\xi, N, R^{\prime}\right)$ and hence $x \in U_{O}\left(\xi, N, R^{\prime}\right) \subset U$.

Now we show that for $[\rho] \in V \cap \partial_{O^{\prime}} X$ we have that $\Phi_{O^{\prime} O}([\rho]) \in U$. Let $\rho=$ $\left(w^{0}=O^{\prime}, w^{1}, w^{2}, \ldots\right) \in \mathcal{G}_{O^{\prime}}(\eta, N+d, R) \cap \mathcal{R}_{O^{\prime}}$. Let $\zeta=\left(w_{0}=O, w_{1}, w_{2}, \ldots\right)$ be obtained from $\rho$ as in the definition of $\Phi_{O^{\prime}} O$. Then, by Lemma 3.9 and Corollary 3.4 , we can perform the same computation as in the previous case to get $\left|w_{N} v_{N}\right| \leq$ $R+3 D+6 d=R^{\prime}$. Thus $\Phi_{O^{\prime} O}([\rho])=[\zeta] \in U_{O}\left(\xi, N, R^{\prime}\right) \subset U$ and we have completed the proof of $\Phi_{O^{\prime} O}(V) \subset U$ and of the whole lemma.

Now we address the question of finite dimensionality of $\bar{X}$. Let us remind that a simplicial complex $X$ is uniformly locally finite if there exists a natural number $L$ such that every vertex belongs to at most $L$ different simplices. This happens for example when some group acts geometrically on $X$. 
Proposition 5.6 Let $X$ be a uniformly locally finite systolic complex. Then $\bar{X}=$ $X \cup \partial_{O} X$ is finitely dimensional.

Proof Recall that a space $Y$ has dimension at most $n$ if, for every open cover $\mathcal{U}$ of $Y$, there exists an open cover $\mathcal{V} \prec \mathcal{U}(\mathcal{V}$ is a refinement of $\mathcal{U}$, ie every element of $\mathcal{V}$ is contained in some element of $\mathcal{U}$ ) such that every point in $Y$ belongs to at most $n+1$ elements of $\mathcal{V}$ (ie the multiplicity of $\mathcal{V}$ is at most $n+1$ ).

It is clear that $X$ is finitely dimensional. It is thus enough to show that there exists a constant $K$ such that for every open (in $\bar{X}$ ) cover $\mathcal{U}$ of $\partial_{O} X$ there exists an open cover $\mathcal{V} \prec \mathcal{U}$ of $\partial_{O} X$ of multiplicity at most $K$.

Let $R>D$ be a natural number. Then, by uniform local finiteness, there is a constant $K$ such that every ball of radius at most $2 R+D+2$ contains at most $K$ vertices.

Let $\mathcal{U}$ be an open cover of $\partial_{O} X$ in $\bar{X}$. We construct an open cover $\mathcal{V} \prec \mathcal{U}$ of $\partial_{O} X$ in $\bar{X}$ consisting of interiors of standard neighborhoods such that the multiplicity of $\mathcal{V}$ is at most $K$.

Let $R^{\prime}=2 R+2 D$. By the definition of topology (Proposition 4.4) and by Lemma 4.3, for every $[\eta] \in \partial_{O} X$ there exists a standard neighborhood $U\left(\eta, N_{\eta}, R^{\prime}\right)$ contained in some element of $\mathcal{U}$. By Lemma 4.8 we have $[\eta] \in \operatorname{int} U\left(\eta, N_{\eta}, R^{\prime}\right)$. By compactness of $\partial_{O} X$ (Proposition 5.3 and Remark 4.5), among such neighborhoods we can find a finite family $\left\{U\left(\eta^{j}, N_{\eta^{j}}, R^{\prime}\right)\right\}_{j=1}^{m}$ such that the family of smaller standard neighborhoods $\left\{U\left(\eta^{j}, N_{\eta^{j}}, R\right)\right\}_{j=1}^{m}$ covers $\partial_{O} X$. Let $N=\max \left\{N_{\eta^{1}}, N_{\eta^{2}}, \ldots, N_{\eta^{m}}\right\}$. Let $A$ denote the set of vertices $v$ in $S_{N}(O)$ for which there exists a good geodesic ray starting at $O$ and passing through $v$. For each $v \in A$, pick some such good geodesic ray $\xi^{v}=\left(w_{0}^{v}=O, w_{1}^{v}, w_{2}^{v}, \ldots, w_{N}^{v}=v, \ldots\right)$. We claim that the family $\mathcal{V}=\left\{\right.$ int $\left.U\left(\xi^{v}, N, R\right) \mid v \in A\right\}$ is as desired.

First we show that $\mathcal{V}$ covers $\partial_{O} X$. Let $\zeta=\left(z_{0}=O, z_{1}, z_{2}, \ldots\right)$ be an arbitrary good

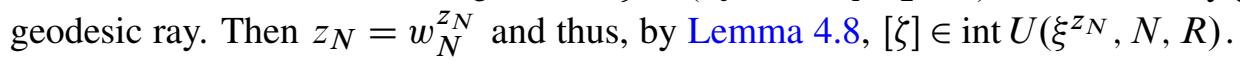

Now we show that $\mathcal{V} \prec \mathcal{U}$. To prove this it is enough to show that for every $v \in A$ there exists $j \in\{1,2, \ldots, m\}$ such that $U\left(\xi^{v}, N, R\right) \subset U\left(\eta^{j}, N_{\eta^{j}}, R^{\prime}\right)$. Let $v \in A$. Choose $j$ such that $\left[\xi^{v}\right] \in U\left(\eta^{j}, N_{\eta^{j}}, R\right)$. By Lemma 4.2, to show that $U\left(\xi^{v}, N, R\right) \subset$ $U\left(\eta^{j}, N_{\eta^{j}}, R^{\prime}\right)$ it is enough to show that, for every $\zeta \in \mathcal{G}\left(\xi^{v}, N, R\right)$, we have $\zeta \in$ $\mathcal{G}\left(\eta^{j}, N_{\eta^{j}}, R^{\prime}\right)$. Let $\zeta=\left(z_{0}=O, z_{1}, z_{2}, \ldots, z_{N}, \ldots\right) \in \mathcal{G}\left(\xi^{v}, N, R\right)$ and denote $\eta^{j}=\left(v_{0}^{j}=O, v_{1}^{j}, v_{2}^{j}, \ldots\right)$. By Lemma 3.8, we have $\left|w_{N_{\eta^{j}}}^{v} v_{N_{\eta^{j}}}^{j}\right| \leq R+D$. Then, by Corollary 3.4, we have

$$
\begin{aligned}
\left|z_{N_{\eta^{j}}} v_{N_{\eta^{j}}}^{j}\right| & \leq\left|z_{N_{\eta^{j}}} w_{N_{\eta^{j}}}^{v}\right|+\left|w_{N_{\eta^{j}}}^{v} v_{N_{\eta^{j}}}^{j}\right| \leq\left(\left|z_{N} w_{N}^{v}\right|+D\right)+(R+D) \\
& \leq 2 R+2 D=R^{\prime} .
\end{aligned}
$$


Thus $\zeta \in \mathcal{G}\left(\eta^{j}, N_{\eta^{j}}, R^{\prime}\right)$ and it follows that $\mathcal{V} \prec \mathcal{U}$.

Finally, we claim that the multiplicity of $\mathcal{V}$ is at most $K$. By Lemma 4.9, if $\left|v v^{\prime}\right|>$ $2 R+D+2$ then int $U\left(\xi^{v}, N, R\right) \cap \operatorname{int} U\left(\xi^{v^{\prime}}, N, R\right) \subset U\left(\xi^{v}, N, R\right) \cap U\left(\xi^{v^{\prime}}, N, R\right)=\varnothing$. Thus multiplicity of $\mathcal{V}$ is at most the number of vertices in a ball of radius $2 R+D+2$ in $X$, ie it is at most $K$.

\section{The main result}

The aim of this section is to prove the main result of the paper-Theorem A (Theorem 6.3).

The following result will be crucial.

Proposition 6.1 [4, Proposition 2.1; 3, Lemma 1.3] Let $(Y, Z)$ be a pair of finitedimensional compact metrizable spaces with $Z$ nowhere dense in $Y$, and such that $Y \backslash Z$ is contractible and locally contractible and the following condition holds:

For every $z \in Z$ and every open neighborhood $U$ of $z$ in $Y$, there exists an open neighborhood $V$ of $z$ contained in $U$ such that $V \backslash Z \hookrightarrow U \backslash Z$ is null-homotopic.

Then $Y$ is an ER and $Z$ is a $Z$-set in $Y$.

Before proving Theorem A we need an important preparatory lemma.

Lemma 6.2 Let $[\eta] \in \partial_{O} X$ and let $U(\eta, N, R)$ be a standard neighborhood of $[\eta]$ in $\bar{X}$. Then there exists $N^{\prime}$ such that $U\left(\eta, N^{\prime}, R\right) \subset U(\eta, N, R)$ and the inclusion map $U\left(\eta, N^{\prime}, R\right) \cap X \hookrightarrow U(\eta, N, R) \cap X$ is null-homotopic.

Proof Let $R^{\prime}=4 D+7$. By Lemma 4.3, there exists $\tilde{N}$ such that $U\left(\eta, \tilde{N}, R^{\prime}\right) \subset$ $U(\eta, N, R)$, so that it is enough to prove the following. For natural $R \geq D$ there exists $N^{\prime}$ such that $U\left(\eta, N^{\prime}, R\right) \subset U\left(\eta, N, R^{\prime}\right)$ and the inclusion map $U\left(\eta, N^{\prime}, R\right) \cap X \hookrightarrow$ $U\left(\eta, N, R^{\prime}\right) \cap X$ is null-homotopic.

Before we start, let us give a rough idea of the proof. Let us restrict to the problem of contracting loops from $U\left(\eta, N^{\prime}, R\right) \cap X$ in $U\left(\eta, N, R^{\prime}\right) \cap X$ (this turns out to be the most complicated case). Let $\alpha$ be such a loop. We connect each vertex of $\alpha$ by a good geodesic with $O$, and we are interested in the vertex of this geodesic at certain distance $M$ from $O$, where $N<M<N^{\prime}$. All vertices constructed in this way lie in 
a certain ball (see Condition 1 below), which is in turn contained in $U\left(\eta, N, R^{\prime}\right) \cap X$ (see Condition 3 below). If we connect these vertices by 1 -skeleton geodesics in the right order, we obtain a loop $\alpha_{M}$, which lies in the ball considered (Corollary 2.7) and is contractible inside this ball (Corollary 2.5). So we need to find a free homotopy between $\alpha$ and $\alpha_{M}$, which we construct via intermediate loops $\alpha_{l}$. To find that two such consecutive loops are homotopic in $U\left(\eta, N, R^{\prime}\right) \cap X$, we need Condition 2. This condition guarantees that all relatively small loops by which consecutive $\alpha_{l}$ differ can be contracted inside $U\left(\eta, N, R^{\prime}\right) \cap X$.

Let $M=N+R+1$ and $N^{\prime}-1 \geq(R+D+4) M$. We will show that $N^{\prime}$ is as desired. Denote $\eta=\left(v_{0}=O, v_{1}, v_{2}, \ldots\right)$. The choice of $M$ and $N^{\prime}$ guarantees that the following three conditions hold.

Condition 1 Let $\xi=\left(w_{0}=O, w_{1}, \ldots, w_{k}\right)$ be a good geodesic with $k \geq N^{\prime}-1$ and $w_{k} \in \overline{U\left(\eta, N^{\prime}, R\right) \cap X}$. Then $w_{M} \in B_{D+1}\left(v_{M}\right)$.

Indeed, let $\left(z_{0}=O, z_{1}, \ldots, z_{l}\right) \in \mathcal{G}\left(\eta, N^{\prime}, R\right)$ be such that $\left|w_{k} z_{l}\right| \leq 1$ (guaranteed by the definition of $U\left(\eta, N^{\prime}, R\right)$ ). Since $k \geq N^{\prime}-1$, we have, by Corollary 3.5, that

$$
\begin{aligned}
\left|w_{N^{\prime}-1} v_{N^{\prime}-1}\right| & \leq\left|w_{N^{\prime}-1} z_{N^{\prime}-1}\right|+\left|z_{N^{\prime}-1} v_{N^{\prime}-1}\right| \\
& \leq\left(2\left|w_{k} z_{l}\right|+D\right)+\left(1+\left|z_{N^{\prime}} v_{N^{\prime}}\right|+1\right) \leq R+D+4 .
\end{aligned}
$$

Thus, by Corollary 3.4, we have

$$
\left|w_{M} v_{M}\right| \leq \frac{M}{N^{\prime}-1}\left|w_{N^{\prime}-1} v_{N^{\prime}-1}\right|+D \leq \frac{1}{R+D+4}\left|w_{N^{\prime}-1} v_{N^{\prime}-1}\right|+D \leq D+1 .
$$

Condition 2 Let $\xi=\left(w_{0}=O, w_{1}, \ldots, w_{k}\right)$ be as in Condition 1. Then, for every $k \geq l \geq M+1$ we have $B_{D+3}\left(w_{l}\right) \subset U\left(\eta, N, R^{\prime}\right) \cap X$.

To show this observe that, as in the proof of Condition 1, we have that $\left|w_{N^{\prime}-1} v_{N^{\prime}-1}\right| \leq$ $R+D+4$. Now, let $z$ be a vertex of $B_{D+3}\left(w_{l}\right)$. Choose a good geodesic $\left(z_{0}=O, z_{1}, z_{2}, \ldots, z_{m}=z\right)$ (guaranteed by Corollary 3.3). Since $l \geq M+1=$ $N+R+2 \geq N+(D+3)$, we have that $m \geq N$ and $z_{N}$ is defined. Thus, by Corollary 3.4 and Corollary 3.5, we have

$$
\begin{aligned}
\left|z_{N} v_{N}\right| & \leq\left|z_{N} w_{N}\right|+\left|w_{N} v_{N}\right| \leq\left(2\left|z_{m} w_{l}\right|+D\right)+\left(\frac{N}{N^{\prime}-1}\left|w_{N^{\prime}-1} v_{N^{\prime}-1}\right|+D\right) \\
& <(2(D+3)+D)+\left(\frac{1}{R+D+4}(R+D+4)+D\right)=4 D+7=R^{\prime} .
\end{aligned}
$$

Thus $z \in U\left(\eta, N, R^{\prime}\right) \cap X$ and it follows that $B_{D+3}\left(w_{l}\right) \subset U\left(\eta, N, R^{\prime}\right) \cap X$. 
Condition 3 We have $B_{D+1}\left(v_{M}\right) \subset U\left(\eta, N, R^{\prime}\right) \cap X$.

This follows immediately from Condition 2, but we want to record it separately.

The goal First observe that $U\left(\eta, N^{\prime}, R\right) \subset U\left(\eta, N, R^{\prime}\right)$ by Lemma 4.3 and the definition of $N^{\prime}$. Now we show that the map $\pi_{i}\left(U\left(\eta, N^{\prime}, R\right) \cap X\right) \rightarrow \pi_{i}\left(U\left(\eta, N, R^{\prime}\right) \cap X\right)$ induced by inclusion is trivial, for every $i=0,1,2, \ldots$. Let $A$ be the smallest full subcomplex of $X$ containing $U\left(\eta, N^{\prime}, R\right) \cap X$. Observe that the vertices of $A$ lie in $\overline{U\left(\eta, N^{\prime}, R\right) \cap X}$. By Condition 2, $A$ is contained in $U\left(\eta, N, R^{\prime}\right) \cap X$. Thus it is enough to show that the map $\pi_{i}(A) \rightarrow \pi_{i}\left(U\left(\eta, N, R^{\prime}\right) \cap X\right)$ induced by the inclusion is trivial and we may restrict ourselves only to simplicial spherical cycles.

Case $(i=0)$ Let $z^{1}, z^{2}$ be two vertices of $A$. We will construct a simplicial path in $U\left(\eta, N, R^{\prime}\right) \cap X$ connecting $z^{1}$ and $z^{2}$.

Choose (using Corollary 3.3) good geodesics $\left(z_{0}^{j}=O, z_{1}^{j}, \ldots, z_{k(j)}^{j}=z^{j}\right), j=1,2$. By Condition 2, $\left(z_{M}^{j}, z_{M+1}^{j}, \ldots, z_{k(j)}^{j}=z^{j}\right)$ is contained in $U\left(\eta, N, R^{\prime}\right)$ and we have $z_{M}^{j} \in B_{D+1}\left(v_{M}\right)$ by Condition 1 . Choose a $1-$ skeleton geodesic $\left(u_{1}=z_{M}^{1}, u_{2}, \ldots\right.$, $u_{l}=z_{M}^{2}$ ). Since balls are geodesically convex (Corollary 2.7$)$, this geodesic is contained in $B_{D+1}\left(v_{M}\right)$ and hence, by Condition 3, it is contained in $U\left(\eta, N, R^{\prime}\right) \cap X$.

Then the 1-skeleton path

$$
\left(z^{1}=z_{k(1)}^{1}, z_{k(1)-1}^{1}, \ldots, z_{M}^{1}=u_{1}, u_{2}, \ldots, u_{l}=z_{M}^{2}, z_{M+1}^{2}, \ldots, z_{k(2)}^{2}=z^{2}\right)
$$

connects $z^{1}$ and $z^{2}$ and is contained in $U\left(\eta, N, R^{\prime}\right) \cap X$. Therefore the map $\pi_{0}(A) \rightarrow$ $\pi_{0}\left(U\left(\eta, N, R^{\prime}\right) \cap X\right)$ is trivial.

Case $(i=1)$ Let $\alpha=\left(z^{0}, z^{1}, \ldots, z^{n}=z^{0}\right)$ be a 1 -skeleton loop in $A$. We show that this loop can be contracted within $U\left(\eta, N, R^{\prime}\right) \cap X$.

Choose good geodesics $\left(z_{0}^{j}=O, z_{1}^{j}, \ldots, z_{k(j)}^{j}=z^{j}\right)$ (guaranteed by Corollary 3.3), for $j=0,1,2, \ldots, n-1$. By $z_{k}^{j}$, for $k>k(j)$, we denote $z^{j}$. Let $K$ be the maximum of $\{k(0), k(1), \ldots, k(n-1)\}$. Observe that, by Corollary 3.5, we have $\left|z_{l}^{j} z_{l}^{j+1}\right| \leq$ $D+2$ (we consider $j$ modulo $n$ ), for every $l=M, M+1, M+2, \ldots, K$ (we are not interested in smaller $l)$. For these $l$ let $\left(z_{l}^{j}=t_{l}^{j, 0}, t_{l}^{j, 1}, \ldots, t_{l}^{j, p_{l}(j)}=z_{l}^{j+1}\right)$ be arbitrary 1 -skeleton geodesics. Record that $p_{l}(j) \leq D+2$.

Thus, for every $l=M+1, M+2, \ldots, K$ and for every $j=0,1, \ldots, n-1$, we have a 1 -skeleton loop

$$
\begin{aligned}
\gamma_{l}^{j}=\left(z_{l}^{j}, z_{l-1}^{j}=t_{l-1}^{j, 0}, t_{l-1}^{j, 1}, \ldots, t_{l-1}^{j, p_{l-1}(j)}\right. & =z_{l-1}^{j+1}, \\
z_{l}^{j+1} & \left.=t^{j, p_{l}(j)}, t_{l}^{j, p_{l}(j)-1}, \ldots, t_{l}^{j, 0}=z_{l}^{j}\right)
\end{aligned}
$$


of length at most

$$
1+p_{l-1}(j)+1+p_{l}(j) \leq 1+(D+2)+1+(D+2)=2 D+6 .
$$

Hence $\gamma_{l}^{j} \subset B_{D+3}\left(z_{l}^{j}\right)$. Since balls are contractible (Corollary 2.5), $\gamma_{l}^{j}$ is contractible inside $B_{D+3}\left(z_{l}^{j}\right)$, which is, by Condition 2 , contained in $U\left(\eta, N, R^{\prime}\right)$. Thus, for $M \leq l \leq K$, the loops

$$
\begin{array}{r}
\alpha_{l}=\left(z_{l}^{0}=t_{l}^{0,0}, t_{l}^{0,1}, \ldots, t_{l}^{0, p_{l}(0)}=z_{l}^{1}=t_{l}^{1,0}, t_{l}^{1,1}, \ldots, t_{l}^{1, p_{l}(1)}=z_{l}^{2}, \ldots\right. \\
\left.\ldots, z_{l}^{n-1}=t_{l}^{n-1,0}, t_{l}^{n-1,1}, \ldots, t_{l}^{n-1, p_{l}(n-1)}=z_{l}^{n}=z_{l}^{0}\right)
\end{array}
$$

for consecutive $l$ are freely homotopic in $U\left(\eta, N, R^{\prime}\right)$.

Observe that $\alpha=\alpha_{K}$. On the other hand $\alpha_{M} \subset B_{D+1}\left(v_{M}\right)$, by Condition 1 and by geodesic convexity of balls (Corollary 2.7). Moreover, since balls are contractible (Corollary 2.5), $\alpha_{M}$ can be contracted inside $B_{D+1}\left(v_{M}\right)$, which lies in $U\left(\eta, N, R^{\prime}\right)$, by Condition 3. Thus $\alpha$ is contractible in $U\left(\eta, N, R^{\prime}\right)$. It follows that the map $\pi_{1}(A) \rightarrow \pi_{1}(U(\eta, N, R) \cap X)$ is trivial.

Case ( $i>1$ ) Since $A$ is a full subcomplex of a systolic complex it is, by Lemma 2.12, aspherical and thus $\pi_{i}(A)=0$ and the map in question is obviously trivial. $\square$

Theorem 6.3 (Theorem A) Let a group $G$ act geometrically by simplicial automorphisms on a systolic complex $X$. Then $\bar{X}=X \cup \partial_{O} X$, where $O$ is a vertex of $X$, is a compactification of $X$ satisfying the following:

(1) $\bar{X}$ is a Euclidean retract (ER).

(2) $\partial_{O} X$ is a $Z$-set in $\bar{X}$.

(3) For every compact set $K \subset X,(g K)_{g \in G}$ is a null sequence.

(4) The action of $G$ on $X$ extends to an action, by homeomorphisms, of $G$ on $\bar{X}$.

Proof (1) and (2) By Corollary 5.2, Proposition 5.3, Remark 4.5, and Proposition 5.6 we have that $\left(\bar{X}, \partial_{O} X\right)$ is a pair of finite-dimensional compact metrizable spaces.

Since $X$ is a simplicial complex, it is locally contractible and, by Theorem 2.3, it is contractible since it is a finitely dimensional systolic complex. By the definition of the topology on $\bar{X}$ (cf Proposition 4.4), it is clear that $\partial_{O} X$ is nowhere dense in $\bar{X}$. Thus we are in a position to apply Proposition 6.1. Let $x \in \partial_{O} X$ and let $U$ be its open neighborhood in $\bar{X}$.

By the definition of the topology (Proposition 4.4) we can find a standard neighborhood $U(\eta, N, R) \subset U$, where $[\eta]=x$. By Lemma 6.2, there exists a standard neighborhood 
$U\left(\eta, N^{\prime}, R\right) \subset U(\eta, N, R) \subset U$ (with $[\eta] \in \operatorname{int}\left(U\left(\eta, N^{\prime}, R\right)\right.$, by Lemma 4.8) such that the map int $\left(U\left(\eta, N^{\prime}, R\right) \cap X\right) \hookrightarrow U\left(\eta, N^{\prime}, R\right) \cap X \hookrightarrow U(\eta, N, R) \cap X \hookrightarrow U \cap X$ is null-homotopic. Thus $\bar{X}$ is an ER and $\partial_{O} X$ is a $Z$-set in $\bar{X}$.

(3) Let $\mathcal{U}$ be an open cover of $\bar{X}$ and let $K \subset X$ be a compact set. We will show that all but finitely many translates $g K$, for $g \in G$, are $\mathcal{U}$-small.

Let $R>D$ be such that $K \subset B_{R}(z)$, for some vertex $z$. As in the proof of Proposition 5.6, we can find a natural number $N$, a finite set of vertices $A \subset S_{N}(O)$ and a collection of good geodesic rays $\left\{\xi^{v} \mid v \in A\right\}$ with $\xi^{v}$ passing through $v$ such that the following holds. The family $\mathcal{V}=\left\{\operatorname{int} U\left(\xi^{v}, N, R\right) \mid v \in A\right\}$ covers $\partial_{O} X$ and the family $\mathcal{V}^{\prime}=\left\{U\left(\xi^{v}, N, 4 R\right) \mid v \in A\right\}$ is a refinement of $\mathcal{U}$. Thus we can find an open cover $\mathcal{W}=\mathcal{V} \cup \mathcal{W}^{\prime}$ of $\bar{X}$ such that every $W \in \mathcal{W}^{\prime}$ is contained in $X$. By compactnessProposition 5.3-there is a finite subfamily of $\mathcal{W}$ covering $\bar{X}$. It follows that there exists a natural number $N^{\prime}>N$ such that $\bar{X} \backslash B_{N^{\prime}}(O) \subset \bigcup \mathcal{V}$. By properness of the action there exists a cofinite subset $H \subset G$ such that $g K \subset B_{R}(g z) \subset X \backslash B_{N^{\prime}}(O)$, for $g \in H$.

We claim that, for every $g \in H$, we have $g K \subset B_{R}(g z) \subset U\left(\xi^{v}, N, 4 R\right) \cap X$, for some $v \in A$. Assertion (3) follows then from the claim. Let $g \in H$. Since $\bar{X} \backslash B_{N^{\prime}}(O) \subset \bigcup \mathcal{V}$, there exists $v \in A$ such that $g z \in \operatorname{int} U\left(\xi^{v}, N, R\right)$. We show that $B_{R}(g z) \subset U\left(\xi^{v}, N, 4 R\right)$. Let $x \in B_{R}(g z)$ and let $\zeta=\left(z_{0}^{\prime}=O, z_{1}^{\prime}, \ldots, z_{l}^{\prime}\right)$ be a good geodesic (which exists by Corollary 3.3) such that $z_{l}^{\prime} \in B_{R}(g z)$ is a vertex of the simplex containing $x$ in its interior. Since $g z \in U\left(\xi^{v}, N, R\right)$ there exists a good geodesic $\left(z_{0}=O, z_{1}, z_{2}, \ldots, z_{k}=g z\right)$, such that $\left|z_{N} v\right| \leq R$. We have $l, k \geq N^{\prime}$ and $\left|z_{l}^{\prime} z_{k}\right| \leq R$. Hence, by Corollary 3.5, we have

$$
\begin{aligned}
\left|z_{N}^{\prime} v\right| & \leq\left|z_{N}^{\prime} z_{N}\right|+\left|z_{N} v\right| \leq\left(2\left|z_{l}^{\prime} z_{k}\right|+D\right)+\left|z_{N} v\right| \\
& \leq(2 R+D)+R<4 R .
\end{aligned}
$$

Thus $\zeta \in \mathcal{G}\left(\xi^{v}, N, 4 R\right)$ and hence $x \in U\left(\xi^{v}, N, 4 R\right)$. It follows that $B_{R}(g z) \subset$ $U\left(\xi^{v}, N, 4 R\right)$. Since $g \in H$ was arbitrary we have that elements of $(g K)_{g \in H}$ are $\mathcal{V}^{\prime}$-small and thus they are $\mathcal{U}$-small.

(4) For $g \in G$ we define a map $g \circ: X \cup \partial_{O} X \rightarrow X \cup \partial_{g O} X$ in the following way. For $x \in X$ let $g \circ x=g x$ and for $x=\left[\left(v_{0}=O, v_{1}, v_{2}, \ldots\right)\right] \in \partial_{O} X$ let $g \circ x=$ $\left[\left(g v_{0}=g O, g v_{1}, g v_{2}, \ldots\right)\right] \in \partial_{g} O X$. This is obviously a well defined homeomorphism. We extend the action of $G$ on $X$ to $X \cup \partial_{O} X$ by the formula $g \cdot x=\Phi_{g O O}(g \circ x)$, for $x \in \partial_{O} X$. By Lemma 5.5, the map $g \cdot: X \cup \partial_{O} X \rightarrow X \cup \partial_{O} X$ is a homeomorphism. To see that $(g h) \cdot x=g \cdot(h \cdot x)$, for $x \in \partial_{O} X$, pick some representative $\eta=\left(v_{0}=O, v_{1}, \ldots\right)$ of $x$. We need to show that

$$
\Phi_{g h O O}(g h \circ[\eta])=\Phi_{g O O}\left(g \circ \Phi_{h O O}(h \circ[\eta])\right) .
$$


Recall that, by Lemma 3.9, mappings $\Phi_{g O O}, \Phi_{h O O}$ and $\Phi_{g h O O}$ displace representative rays by a finite Hausdorff distance. Hence $\Phi_{g h O O}(g h \circ[\eta])$ is the class of rays starting at $O$ at a finite Hausdorff distance from $\left(g h v_{0}=g h O, g h v_{1}, \ldots\right)$. On the other hand, $\Phi_{h O O}(h \circ[\eta])$ is the class of rays starting at $O$ at a finite Hausdorff distance from $\left(h v_{0}=h O, h v_{1}, \ldots\right)$, hence $g \circ \Phi_{h O O}(h \circ[\eta])$ as well as $\Phi_{g O O}\left(g \circ \Phi_{h O O}(h \circ[\eta])\right)$ is the class of rays (starting at $g O$ and $O$, respectively) at a finite Hausdorff distance from $\left(g h v_{0}=g h O, g h v_{1}, \ldots\right)$. This proves the desired equality.

Hence we get an extension of the action of $G$ on $X$ to an action on $\bar{X}$ by homeomorphisms.

\section{Flat surfaces}

With this section we start the second part of the article, in which we define Euclidean geodesics, establish Theorem B and Theorem C. Before we define Euclidean geodesics, we first need to study, as mentioned in Section 1, the minimal surface spanned on a pair of directed geodesics connecting given vertices. The tools for this are minimal surfaces (Section 7) and layers (Section 8).

In this section we recall some definitions and facts concerning flat minimal surfaces in systolic complexes proved by Elsner [14; 15].

Definition 7.1 The flat systolic plane is a systolic 2-complex obtained by equilaterally triangulating the Euclidean plane. We denote it by $\mathbb{E}_{\Delta}^{2}$. A systolic disc is a systolic triangulation of a 2-disc and a flat disc is any systolic disc $\Delta$, which can be embedded into $\mathbb{E}_{\Delta}^{2}$, such that $\Delta^{(1)}$ is embedded isometrically into the 1 -skeleton of $\mathbb{E}_{\Delta}^{2}$. A systolic disc $\Delta$ is called wide if $\partial \Delta$ is a full subcomplex of $\Delta$. For any vertex $v \in \Delta^{(0)}$ the defect at $v$ (denoted by $\operatorname{def}(v)$ ) is $6-t(v)$ for $v \notin \partial \Delta^{(0)}$, and $3-t(v)$ for $v \in \partial \Delta^{(0)}$, where $t(v)$ is the number of triangles in $\Delta$ containing $v$. It is clear that internal vertices of a systolic disc have nonpositive defects.

We will need the following easy and well known fact.

Lemma 7.2 (Gauss-Bonnet Lemma) If $\Delta$ is any triangulation of a 2-disc, then

$$
\sum_{v \in \Delta^{(0)}} \operatorname{def}(v)=6
$$

Flat systolic discs can be characterized as follows. 
Lemma 7.3 [15, Lemma 2.5] A systolic disc $D$ is flat if and only if it satisfies the following three conditions:

(i) $D$ has no internal vertices of defect $<0$.

(ii) $D$ has no boundary vertices of defect $<-1$.

(iii) Any segment in $\partial D$ connecting vertices with defect $<0$ contains a vertex of defect $>0$.

Now we recall another handful of definitions.

Definition 7.4 Let $X$ be a systolic complex. Any simplicial map $S: \Delta \rightarrow X$, where $\Delta$ is a triangulation of a $2-$ disc, is called a surface. We say that $S$ is spanned on a loop $\gamma$, if $\left.S\right|_{\partial \Delta}=\gamma$. A loop $\gamma$ is triangulable, if there exists a surface $S$ spanned on $\gamma$, such that all the vertices of $\Delta$ are in $\partial \Delta$. A surface $S$ is systolic, flat or wide if the disc $\Delta$ satisfies the corresponding property. If $S$ is injective on $\partial \Delta$ and minimal (the smallest number of triangles in $\Delta$ ) among surfaces with the given image of $\partial \Delta$, then $S$ is called minimal. A geodesic in $\Delta^{(1)}$ is called neat if it stays out of $\partial \Delta$ except possibly at its endpoints. A surface $S$ is called almost geodesic if it maps neat geodesics in $\Delta^{(1)}$ isometrically into $X^{(1)}$.

The following is part of the main theorem of Elsner [15].

Theorem 7.5 [15, Theorem 3.1] Let $X$ be a systolic complex. If $S$ is a wide flat minimal surface in $X$ then $S$ is almost geodesic.

We will also use the following handy fact, whose proof can be extracted from Elsner [14]. In case where $\gamma$ has length 2 , it follows immediately from 6 -largeness.

Proposition 7.6 [14, Proposition 3.10] Let $X$ be a systolic complex and $S: \Delta \rightarrow X$ a wide flat minimal surface. Let $\gamma$ be a neat 1 -skeleton geodesic in $\Delta \subset \mathbb{E}_{\Delta}^{2}$, which is contained in a straight line. Then, for any 1 -skeleton geodesic $\bar{\gamma}$ in $X$ with the same endpoints as $S(\gamma)$, there is another minimal surface $S^{\prime}: \Delta \rightarrow X$ such that $S^{\prime}(\gamma)=\bar{\gamma}$ and $S=S^{\prime}$ on the vertices of $\Delta$ outside $\gamma$.

\section{Layers}

In this section we introduce and study the notion of layers for a pair of convex subcomplexes of a systolic complex. If those subcomplexes are vertices $v, w$, then the layer $k$ is the span of all vertices, in 1 -skeleton geodesics $v w$, at distance $k$ from $v$ 
(cf Definition 8.1). In particular, simplices of the directed geodesics between $v$ and $w$ (cf Definition 2.11), as well as the simplices of Euclidean geodesics (which we construct in Section 9) lie in appropriate layers.

On the other hand, layers in systolic complexes seem to be interesting on their own.

Definition 8.1 Let $V, W$ be convex subcomplexes of a systolic complex $X$ and $n=|V, W|$. For $i=0,1, \ldots, n$ we define the layer $i$ between $V$ and $W$ as the subcomplex of $X$ equal to $B_{i}(V) \cap B_{n-i}(W)$. We denote it by $L_{i}(V, W)$ (or shortly $L_{i}$ when $V, W$ are understood).

Remark 8.2 $L_{i}$ are convex, since they are intersections of convex $B_{i}(V), B_{n-i}(W)$ (see remarks after Definition 2.4).

Lemma $8.3 \quad$ (i) $L_{i}=S_{i}(V) \cap S_{n-i}(W)$, for $0 \leq i \leq n$.

(ii) $L_{j} \subset S_{j-i}\left(L_{i}\right)$, for $0 \leq i<j \leq n$. In particular $L_{i+1} \subset S_{1}\left(L_{i}\right)$, for $0 \leq i<n$.

Proof (i) Without loss of generality, we only need to prove that $L_{i} \subset S_{i}(V)$. Take a vertex $x \in L_{i}$. Then we have $|x, V| \leq i$ and $|x, W| \leq n-i$, while $|V, W|=n$. Thus by the triangle inequality we have $|x, V|=i$, as desired.

(ii) By (i) we have that $L_{j} \cap B_{j-i-1}\left(L_{i}\right)=\varnothing$, thus we only need to prove that $L_{j} \subset B_{j-i}\left(L_{i}\right)$. Let $x$ be a vertex in $L_{j}$. Since, by (i), we have $x \in S_{j}(V)$, there is a vertex $y \in B_{i}(V)$ at distance $j-i$ from $x$. Since $x \in B_{n-j}(W)$, we have $y \in B_{n-i}(W)$. Thus $y \in L_{i}$ and $x \in B_{j-i}\left(L_{i}\right)$.

Now we study the properties of layers.

Lemma 8.4 For $0<i<n$ we have that $L_{i}$ is $\infty$-large.

Proof Suppose the layer $L_{i}$ is not $\infty$-large. Then there exists an embedded cycle $\Gamma$ in $L_{i}$ of length at least 4, which is a full subcomplex of $X$.

Denote $D_{1}=\operatorname{span}\left\{B_{i-1}(V), \Gamma\right\}, D_{2}=\operatorname{span}\left\{B_{n-i-1}(W), \Gamma\right\}$. We have $D_{1} \cap D_{2}=\Gamma$. Notice that $D_{1} \cup D_{2}$ is a full subcomplex of $X$, because there are no edges in $X$ between vertices in $B_{i-1}(V)$ and vertices in $B_{n-i-1}(W)$.

Observe that $\Gamma$ is contractible in $D_{1}$ (and similarly in $D_{2}$ ). Indeed, by Lemma 8.3(i) we have that $\Gamma \subset S_{i}(V)$. Thus we can project the edges of $\Gamma$ onto $B_{i-1}(V)$ (cf Definition 2.9). If we choose a vertex in each of these projections, we get, by Lemma 2.8 , that these vertices form a loop. This loop is homotopic to $\Gamma$ in $D_{1}$. Moreover, since $B_{i-1}(V)$ is contractible (by remarks after Definition 2.4) it follows that $\Gamma$ is 
contractible in $D_{1}$ (and similarly in $D_{2}$ ), as desired. The simplicial sphere $S$ formed of these two contractions is contractible in $D_{1} \cup D_{2}$, since full subcomplexes of $X$ are aspherical (Lemma 2.12).

Now use Mayer-Vietoris sequence of the pair $D_{1}, D_{2}$. Since $[\Gamma]$ is the image of $[S]=0$ under $H_{2}\left(D_{1} \cup D_{2}\right) \rightarrow H_{1}\left(D_{1} \cap D_{2}\right)$ it follows that the cycle $\Gamma$ is homological to zero in itself. This is a contradiction.

Lemma 8.5 Let $\sigma_{1}, \sigma_{2}, \sigma_{3}$ be maximal simplices in the layer $L_{i}$ for some $0 \leq i \leq n$ and $\tau_{1}=\sigma_{1} \cap \sigma_{2}, \tau_{2}=\sigma_{2} \cap \sigma_{3}$. Then $\tau_{1} \cap \tau_{2}=\varnothing$ or $\tau_{1} \subset \tau_{2}$ or $\tau_{2} \subset \tau_{1}$.

Proof Without loss of generality, assume that $i \neq 0$ (but we might have $i=n$ ). Suppose the lemma is false. Then there exist vertices $p_{1} \in \tau_{1} \backslash \tau_{2}, p_{2} \in \tau_{2} \backslash \tau_{1}, r \in$ $\tau_{1} \cap \tau_{2}$. By Lemma 8.3(ii) we have that $\sigma_{1}, \sigma_{3} \subset S_{1}\left(L_{i-1}\right)$. Denote by $q_{1}, q_{2}$ some vertices in the projections (cf Definition 2.9) of $\sigma_{1}, \sigma_{3}$ onto $L_{i-1}$. We have $\left|q_{1} q_{2}\right| \leq 1$, because both $q_{1}$ and $q_{2}$ are neighbors of $r$ and the projection of $r \in L_{i} \subset S_{1}\left(L_{i-1}\right)$ (cf Lemma 8.3(ii)) onto $L_{i-1}$ is a simplex (Lemma 2.8). Now we will argue that we can assume that $q_{1} p_{2}$ is an edge. In case $q_{1} \neq q_{2}$ consider the 4 -cycle $q_{1} q_{2} p_{2} p_{1} q_{1}$. It must have a diagonal. We can then assume without loss of generality that $q_{1} p_{2}$ is an edge. In case $q_{1}=q_{2}$ we also have that $q_{1} p_{2}$ is an edge. In both cases it follows that $p_{2}$ belongs to the simplex which is the projection of $q_{1} \in L_{i-1} \subset S_{1}\left(L_{i}\right)$ (cf Lemma 8.3(ii)) onto $L_{i}$. This simplex also contains $\sigma_{1}$. But $p_{2} \notin \sigma_{1}$, which contradicts the maximality of $\sigma_{1}$.

Corollary 8.6 Let $T$ be the following simplicial complex: the trapezoid built out of the three triangles $p_{1} r s_{1}, p_{1} r p_{2}, p_{2} r s_{2}$. Then there is no isometric embedding of $T^{(1)}$ into $L_{i}^{(1)}$, for $0 \leq i \leq n$.

Proof Extend the images of those three triangles to maximal simplices $\sigma_{1}, \sigma_{2}, \sigma_{3}$ and apply Lemma 8.5 .

Corollary 8.7 Let $0<i<n$. Let $\left|p_{0} r_{0}\right| \leq 1,\left|p_{d} r_{d}\right| \leq 1$ for vertices $p_{0}, r_{0}, p_{d}, r_{d} \in$ $L_{i}$ such that $\left|p_{0} p_{d}\right|=\left|r_{0} r_{d}\right|=d \geq 2$ and $\left|p_{0} r_{d}\right| \geq d,\left|r_{0} p_{d}\right| \geq d$. Then, for any 1 -skeleton geodesics $\left(p_{i}\right),\left(r_{i}\right), 0 \leq i \leq d$, connecting $p_{0}$ with $p_{d}$ and $r_{0}$ with $r_{d}$, respectively, and for any $0 \leq i, j \leq d$ such that $|i-j| \leq 1$, we have that $\left|p_{i} r_{j}\right| \leq 1$ (ie $p_{i} r_{j}$ is an edge or $p_{i}=r_{j}$ ).

Proof We will prove the corollary by induction on $d$. First observe that since $L_{i}$ is $\infty$-large (Lemma 8.4), the loop $p_{0} p_{1} \cdots p_{d} r_{d} \cdots r_{1} r_{0} p_{0}$ is triangulable and there exists a diagonal cutting off a triangle. There are only four possibilities for this diagonal 
and we can without loss of generality assume that this diagonal is $p_{0} r_{1}$. Now since $p_{0} \in S_{d}\left(r_{d} p_{d}\right)$ and both $p_{1}$ and $r_{1}$ lie in the projection of $p_{0}$ onto $B_{d-1}\left(r_{d} p_{d}\right)$, then by Lemma 2.8 either $p_{1} r_{1}$ is an edge or $p_{1}=r_{1}$.

Now we start the induction. If $d=2$ and the loop $p_{1} r_{1} r_{2} p_{2} p_{1}$ is embedded, then it has a diagonal. The rest of the required inequalities follows from applying Corollary 8.6 twice.

Suppose that for $d-1$ the corollary is already proved. Then applying it to the pair $p_{1} r_{1}, p_{d} r_{d}$ yields all the required inequalities except for the estimate on $\left|r_{0} p_{1}\right|$. But this follows from Corollary 8.6 applied to the trapezoid $r_{0} p_{0} r_{1} p_{1} p_{2}$.

Corollary 8.8 If $p r, p^{\prime} r^{\prime}$ are edges in $L_{i}$, for some $0<i<n$, such that $\left|p p^{\prime}\right|=$ $\left|r r^{\prime}\right|=d \geq 2$ and $\left|p r^{\prime}\right| \leq d,\left|p^{\prime} r\right| \leq d$, then $\left|p r^{\prime}\right|=\left|p^{\prime} r\right|=d$.

Proof By contradiction.

Case $\left|p r^{\prime}\right|=\left|p^{\prime} r\right|=d-1 \quad$ If $d>2$ (if $d=2$ there is a diagonal in the square $p r^{\prime} p^{\prime} r p$ ) then Corollary 8.7 applied to $d-1$ in place of $d, p_{0}=p, p_{d-1}=r^{\prime}, r_{0}=r, r_{d-1}=p^{\prime}$ gives $\left|p p^{\prime}\right|=\left|r r^{\prime}\right|=d-1$, which is a contradiction.

Case $\left|p r^{\prime}\right|=d-1,\left|p^{\prime} r\right|=d \quad$ Again apply Corollary 8.7, with $p_{0}=p, r_{0}=r, p_{d}=$ $r_{d}=p^{\prime}, p_{d-1}=r^{\prime}$, getting $\left|r r^{\prime}\right|=d-1$, which is a contradiction.

Below we present another important property of layers. Since it will not be needed in the article, we do not include the proof. Denote $L=\operatorname{span}\left(L_{i} \cup L_{i+1}\right)$ for some $1 \leq i<n-1$.

Lemma 8.9 $L$ is $\infty$-large.

We end with a simple, but useful observation.

Lemma 8.10 For any edges $v w, x y$ such that $v, x \in L_{i}, w, y \in L_{i+1}$, where $0 \leq i<n$, we have that ||$v x|-| w y|| \leq 1$.

Proof By contradiction. Suppose, without loss of generality, that $|w y|=2+|v x|$. Hence $v$ lies on a 1 -skeleton geodesic $w y$. Thus, by convexity of layers (Remark 8.2) and by Proposition 2.6, we have that $v$ lies in $L_{i+1}$, which is, by Lemma 8.3, disjoint with $L_{i}$, contradiction. 


\section{Euclidean geodesics}

In this section we define, for a pair of simplices $\sigma, \tau$ as below, a sequence of simplices in the layers between $\sigma$ and $\tau$, which can be considered as a "Euclidean" geodesic joining $\sigma$ and $\tau$. Unlike the directed geodesics defined by Januszkiewicz and Świątkowski (see Definition 2.11), Euclidean geodesics are symmetric with respect to $\sigma$ and $\tau$.

The definition requires a lengthy preparation. Roughly speaking, we start by spanning a minimal surface between directed geodesics from $\sigma$ to $\tau$ and from $\tau$ to $\sigma$. We observe that this surface is flat whenever the two directed geodesics are far apart (we call the corresponding layers thick). Next we show that this "piecewise" flat surface is in some sense unique. This occupies the first part of the section, up to Definition 9.9. Then we look at the geodesics in the Euclidean metric in the flat pieces and use them to define Euclidean geodesics in systolic complexes, cf Definition 9.12. Finally, we establish some of their basic properties.

The setting, which we fix for Sections 9-13 is the following. Let $\sigma, \tau$ be simplices of a systolic complex $X$, such that for some natural $n \geq 0$ we have $\sigma \subset S_{n}(\tau), \tau \subset S_{n}(\sigma)$. Let $\sigma_{0} \subset \sigma, \sigma_{1}, \ldots, \sigma_{n} \subset \tau$ and $\tau_{n} \subset \tau, \tau_{n-1}, \ldots, \tau_{0} \subset \sigma$ be sequences of simplices in $X$, such that for $0 \leq k<n$ we have that $\sigma_{k}, \sigma_{k+1}$ span a simplex and $\tau_{k}, \tau_{k+1}$ span a simplex. In particular, $\sigma_{k}, \tau_{k}$ lie in the layer $k$ between $\sigma$ and $\tau$ (cf Definition 8.1).

Note that if $\sigma_{0}=\sigma, \sigma_{1}, \ldots, \sigma_{n} \subset \tau$ is the directed geodesic from $\sigma$ to $\tau$ and $\tau_{n}=$ $\tau, \tau_{n-1}, \ldots, \tau_{0} \subset \sigma$ is the directed geodesic from $\tau$ to $\sigma$ (cf Definition 2.11), then the above condition is satisfied. This special choice of $\left(\sigma_{k}\right),\left(\tau_{k}\right)$ will be very important later and we will frequently distinguish it.

Definition 9.1 For $0 \leq i \leq n$ the thickness of the layer $i$ for $\left(\sigma_{k}\right),\left(\tau_{k}\right)$ is the maximal distance between vertices in $\sigma_{i}$ and in $\tau_{i}$. If the layer $i$ for $\left(\sigma_{k}\right),\left(\tau_{k}\right)$ has thickness $\leq 1$ we say that the layer $i$ for $\left(\sigma_{k}\right),\left(\tau_{k}\right)$ is thin, otherwise we say that the layer $i$ for $\left(\sigma_{k}\right),\left(\tau_{k}\right)$ is thick. If $\left(\sigma_{k}\right),\left(\tau_{k}\right)$ are directed geodesics from $\sigma$ to $\tau$ and from $\tau$ to $\sigma$, respectively, then we skip "for $\left(\sigma_{k}\right),\left(\tau_{k}\right)$ " for simplicity.

Caution Perhaps, to avoid confusion, we should not have used the word "layer" in the above definition, since we are in fact only checking the position of $\sigma_{i}$ with respect to $\tau_{i}$. Even if the layer $i$ between $\sigma$ and $\tau$ is large, it can happen that the thickness of the layer $i$ for $\left(\sigma_{k}\right),\left(\tau_{k}\right)$ is small. However, we decided that this terminology suits well our approach, in which we will be mostly interested in the part of the layer $i$ between $\sigma$ and $\tau$, which lies "between" $\sigma_{i}$ and $\tau_{i}$. 
Definition 9.2 A pair $(i, j)$, where $0 \leq i<j \leq n$, is called a thick interval (for $\left(\sigma_{k}\right),\left(\tau_{k}\right)$ ) if the layers $i$ and $j$ (for $\left.\left(\sigma_{k}\right),\left(\tau_{k}\right)\right)$ are thin, $i+1<j$, and for every $l$, such that $i<l<j$, the layer $l$ (for $\left(\sigma_{k}\right),\left(\tau_{k}\right)$ ) is thick. We say that the thick interval $(i, j)$ contains $l$ if $i<l<j$.

Lemma 9.3 (i) The thickness of consecutive layers varies at most by 1 .

(ii) If $(i, j)$ is a thick interval (for $\left.\left(\sigma_{k}\right),\left(\tau_{k}\right)\right)$, then $\sigma_{i}, \tau_{i}$ are disjoint.

Proof Both parts follow immediately from Lemma 8.10.

Definition 9.4 Let $(i, j)$ be a thick interval (for $\left(\sigma_{k}\right),\left(\tau_{k}\right)$ ). Let vertices $s_{k} \in \sigma_{k}, t_{k} \in$ $\tau_{k}$ be such that for each $i \leq k \leq j$ the distance $\left|s_{k} t_{k}\right|$ is maximal (ie $s_{k}, t_{k}$ realize the thickness of the layer $k$ ). The sequence $s_{i}, s_{i+1}, \ldots, s_{j}, t_{j}, t_{j-1}, \ldots t_{i}, s_{i}$ is an embedded loop by Lemma 9.3(ii), thus we can consider a minimal surface $S: \Delta \rightarrow X$ spanned on this loop (cf Definition 7.4). We say that $S$ is a characteristic surface (for the thick interval $(i, j))$ and $\Delta$ is a characteristic disc.

Lemma 9.5 Suppose that the layer $k$ is thick. For $s_{k}, s_{k}^{\prime} \in \sigma_{k}, t_{k}, t_{k}^{\prime} \in \tau_{k}$, if distances $\left|s_{k} t_{k}^{\prime}\right|,\left|s_{k}^{\prime} t_{k}\right|$ equal the thickness of the layer $k$ then also $\left|s_{k} t_{k}\right|$ equals the thickness of the layer $k$, ie if vertices $s_{k} \in \sigma_{k}, t_{k} \in \tau_{k}$ realize the thickness in some pairs, then they also realize the thickness as a pair.

Proof Immediate from the definition of thickness and Corollary 8.8.

The lemma below summarizes the geometry of characteristic discs, which we need to introduce the concept of a Euclidean geodesic. The special features of characteristic discs in the case where $\left(\sigma_{k}\right),\left(\tau_{k}\right)$ are directed geodesics will be given in Lemma 9.16 at the end of this section.

Let $S: \Delta \rightarrow X$ be a characteristic surface. Denote by $v_{k}, w_{k}$ in $\Delta$ the preimages of $s_{k}, t_{k}$ in $X$, respectively. This notation will be fixed for the entire article. Let us point out that we use numbers $i, \ldots, j$ to number the layers in $\Delta$ (cf Definition 8.1) between $v_{i} w_{i}$ and $v_{j} w_{j}$, instead of $0, \ldots j-i$, for the sake of clarity.

Lemma 9.6 (i) $\Delta$ (and thus the characteristic surface $S$ ) is wide and flat,

(ii) If we embed $\Delta \subset \mathbb{E}_{\Delta}^{2}$, then the edges $v_{i} w_{i}$ and $v_{j} w_{j}$ are parallel and consecutive layers between them are contained in consecutive straight lines (treated as subcomplexes of $\mathbb{E}_{\Delta}^{2}$ ) parallel to the lines containing $v_{i} w_{i}$ and $v_{j} w_{j}$. 
Proof (i) To prove wideness it is enough to show that any nonconsecutive vertices of the boundary loop are at distance $\geq 2$. Since the layers $k$, where $i<k<j$, are thick (for $\left(\sigma_{k}\right),\left(\tau_{k}\right)$ ), the only possibility for this to fail is that (without loss of generality) $\left|s_{k} t_{k+1}\right|=1$ for some $i<k<j$. If this happens, then both $s_{k}$ and $t_{k}$ lie in the projection of $t_{k+1}$ onto the layer $k$ between $\sigma$ and $\tau$ (the projection is defined by Lemma 8.3(ii)), hence they are neighbors (Lemma 2.8), which contradicts $\left|s_{k} t_{k}\right| \geq 2$. Thus a characteristic disc is wide.

Before proving flatness, we need the following general observation. If $\Gamma$ is a 1 -skeleton geodesic, which is in the boundary of a triangulation of a disc, then the sum of the defects at the vertices in the interior of $\Gamma$ is at most 1 . Moreover, all the defects at these vertices are at most 1 and each two vertices of positive defect are separated by a vertex of negative defect.

To prove flatness we compute possible defects at the boundary vertices of $\Delta$. By wideness, they are at most 1 at $v_{i}, v_{j}, w_{i}, w_{j}$. Moreover, their sum over the interior vertices of both 1 -skeleton geodesics $\left(v_{k}\right)_{k=i}^{j},\left(w_{k}\right)_{k=i}^{j}$ is at most 1 (they are 1skeleton geodesics, since their images are). Thus Gauss-Bonnet Lemma 7.2 implies that the defects of the interior vertices are equal to zero, the sums of the defects over the vertices $\left(v_{k}\right)_{k=i+1}^{j-1},\left(w_{k}\right)_{k=i+1}^{j-1}$ equal 1 each and the defects at $v_{i}, v_{j}, w_{i}, w_{j}$ are equal to 1 .

We now want to say more about the defects at $\left(v_{k}\right)_{k=i+1}^{j-1}$. Up to now we know that their sum is 1 , they equal $1,0,-1$ or -2 and each two vertices of positive defect are separated by a vertex of negative defect (since $\left(v_{k}\right)_{k=i}^{j}$ is a 1-skeleton geodesic). This implies that the defects equal alternatingly $1,-1,1-1, \ldots, 1$ with possible 0 's between them. The same holds for the defects at $\left(w_{k}\right)_{k=i+1}^{j-1}$. Thus, by Lemma 7.3 (characterization of flatness), the characteristic disc $\Delta$ is flat, ie we have an embedding $\Delta \subset \mathbb{E}_{\Delta}^{2}$ isometric on the 1-skeleton.

(ii) By the computation of defects in the proof of (i) we get that the edges $v_{i} w_{i}$ and $v_{j} w_{j}$ are parallel in $\mathbb{E}_{\Delta}^{2}$. We also get that $v_{k}, w_{k}$, for $i \leq k \leq j$, are at combinatorial distances $k-i, j-k$ from the lines containing the edges $v_{i} w_{i}, v_{j} w_{j}$. Hence $v_{k}, w_{k}$ lie on the appropriate line parallel to $v_{i} w_{i}$ and the vertices of $\Delta$ split into families lying on geodesics $v_{k} w_{k}$. By convexity of layers, Remark 8.2, (or by direct observation) these geodesics are equal to the layers.

When speaking about the layers in $\Delta$ between $v_{i} w_{i}$ and $v_{j} w_{j}$, we will often skip "between $v_{i} w_{i}$ and $v_{j} w_{j}$ ". 
Remark 9.7 Denote the layer $k$ in $\Delta$ (between $v_{i} w_{i}$ and $v_{j} w_{j}$ ) by $L_{k}$. Then $S\left(L_{k}\right)$ is contained in the layer $k$ in $X$ between $\sigma$ and $\tau$. This follows from

$$
\begin{aligned}
S\left(L_{k}\right) & \subset S\left(B_{k-i}\left(v_{i} w_{i}\right)\right) \cap S\left(B_{j-k}\left(v_{j} w_{j}\right)\right) \\
& \subset B_{k-i}\left(S\left(v_{i} w_{i}\right)\right) \cap B_{j-k}\left(S\left(v_{j} w_{j}\right)\right) \\
& \subset B_{k-i}\left(s_{i} t_{i}\right) \cap B_{j-k}\left(s_{j} t_{j}\right) \\
& \subset B_{k-i}\left(B_{i}(\sigma)\right) \cap B_{j-k}\left(B_{n-j}(\tau)\right)=B_{k}(\sigma) \cap B_{n-k}(\tau) .
\end{aligned}
$$

The next lemma summarizes some uniqueness properties of characteristic surfaces for a fixed thick interval $(i, j)$.

Lemma 9.8 (i) A characteristic surface is almost geodesic. In particular, it is an isometric embedding on the 1-skeleton of a subcomplex spanned by any pair of consecutive layers between $v_{i} w_{i}$ and $v_{j} w_{j}$ in $\Delta$.

(ii) A characteristic disc $\Delta \subset \mathbb{E}_{\Delta}^{2}$ does not depend (up to isometry) on the choice of $s_{k}, t_{k}$ and the choice of a characteristic surface.

If we have two characteristic surfaces $S_{1}: \Delta_{1} \rightarrow X, S_{2}: \Delta_{2} \rightarrow X$, then after identifying the characteristic discs $\Delta_{1}=\Delta_{2}$ (which is possible by (ii)) we have that

(iii) for any vertices $x, y \in \Delta_{1}=\Delta_{2}$ at distance $1, S_{1}(x)$ and $S_{2}(y)$ are also at distance 1 , ie for any two characteristic surfaces $S_{1}, S_{2}$ we can substitute an image of a vertex of the first surface with the corresponding image in the second and get another characteristic surface,

(iv) for any vertex $x \in \Delta_{1}=\Delta_{2}, S_{1}(x)$ and $S_{2}(x)$ are at distance at most 1 .

Proof (i) This follows from Elsner's Theorem 7.5, since, by Lemma 9.6(i), a characteristic disc is flat and wide. The second part follows from the fact that any two vertices in a same or consecutive layers in $\Delta \subset \mathbb{E}_{\Delta}^{2}$ can be connected by a neat geodesic, which can be verified by direct observation.

(ii) Observe that, by Lemma 9.6(ii), the isometry class of $\Delta$ is determined by the distances $\left|v_{k} w_{k}\right|,\left|v_{k} w_{k+1}\right|$, for $i \leq k \leq j-1$, which are equal, by (i), to $\left|s_{k} t_{k}\right|,\left|s_{k} t_{k+1}\right|$, respectively. The value $\left|s_{k} t_{k}\right|$ equals the thickness of the layer $k$, so it does not depend on the choices. To prove the same for $\left|s_{k} t_{k+1}\right|$, consider two characteristic surfaces constructed for choices $s_{l}, s_{l}^{\prime} \in \sigma_{l}, t_{l}, t_{l}^{\prime} \in \tau_{l}$, where $l=k, k+1$. We will prove that $\left|s_{k} t_{k+1}\right|=\left|s_{k} t_{k+1}^{\prime}\right|=\left|s_{k}^{\prime} t_{k+1}^{\prime}\right|$. We restrict ourselves to proving the first equality (the second is proved analogously). By Lemma 9.5 we have that $\left|s_{k+1} t_{k+1}^{\prime}\right|$ is the thickness of the layer $k+1$. Thus there is a characteristic surface spanned on a loop passing through $s_{k}, t_{k}, s_{k+1}, t_{k+1}^{\prime}$. Hence, by (i), the distance $\left|s_{k} t_{k+1}^{\prime}\right|$ is determined by $\left|s_{k} t_{k}\right|$ and $\left|s_{k+1} t_{k}\right|$, thus it is the same as $\left|s_{k} t_{k+1}\right|$, as desired. 
(iii) If $x$ and $y$ are both boundary vertices, then this is obvious. Otherwise, without loss of generality assume that $x$ is an interior vertex of $\Delta$. Suppose that $x$ lies in the layer $k$ (we denote it by $L_{k}$ ) in $\Delta$ between $v_{i} w_{i}$ and $v_{j} w_{j}$. Denote the thickness of the layer $k$ for $\left(\sigma_{t}\right),\left(\tau_{t}\right)$ by $d$.

First consider the case where $y \in L_{k}$. By Remark 9.7 we have that $S_{1}\left(L_{k}\right)$ and $S_{2}\left(L_{k}\right)$ lie in the layer $k$ in $X$ between $\sigma$ and $\tau$. By Lemma 9.5 we have that $\left|S_{2}\left(v_{k}\right) S_{1}\left(w_{k}\right)\right|=\left|S_{1}\left(v_{k}\right) S_{2}\left(w_{k}\right)\right|=d$. Hence Corollary 8.7 applied to $S_{1}\left(L_{k}\right)$ and $S_{2}\left(L_{k}\right)$ gives $\left|S_{1}(x) S_{2}(y)\right|=1$, as desired.

Now, without loss of generality, consider the remaining case that $y$ is in the layer $k-1$ (denoted by $L_{k-1}$ ) in $\Delta$ between $v_{i} w_{i}$ and $v_{j} w_{j}$. Denote by $y^{\prime}, x^{\prime \prime}$ the common neighbors of $x, y$ in $L_{k-1}, L_{k}$, respectively, and by $x^{\prime}$ the neighbor of $x$ in $L_{k}$ different from $x^{\prime \prime}$. Then we have that $S_{1}(x) S_{2}\left(x^{\prime}\right) S_{2}\left(y^{\prime}\right) S_{2}(y) S_{2}\left(x^{\prime \prime}\right) S_{1}(x)$ is a loop of length 5 from the previous case, hence it is triangulable. By (i), all $\left|S_{2}\left(x^{\prime}\right) S_{2}\left(x^{\prime \prime}\right)\right|,\left|S_{2}\left(x^{\prime}\right) S_{2}(y)\right|,\left|S_{2}\left(x^{\prime \prime}\right) S_{2}\left(y^{\prime}\right)\right|$ equal 2, hence $\left|S_{1}(x) S_{2}(y)\right|=1$, as desired.

Observe that this proof actually implies Proposition 7.6 in the case where $\gamma \subset v_{k} w_{k}$ for some $k$.

(iv) For boundary vertices this is obvious. For an interior vertex $x$, let $x^{\prime}, x^{\prime \prime}$ be its neighbors in a common layer in $\Delta$ between $v_{i} w_{i}, v_{j} w_{j}$. Then, by (iii), we have that $S_{1}(x) S_{2}\left(x^{\prime}\right) S_{2}(y) S_{2}\left(x^{\prime \prime}\right) S_{1}(x)$ is a loop of length 4 . Moreover, by (i), we have that $\left|S_{2}\left(x^{\prime}\right) S_{2}\left(x^{\prime \prime}\right)\right|=2$. Thus $\left|S_{1}(x) S_{2}(y)\right| \leq 1$, as desired.

As a corollary, the following definition is allowed.

Definition 9.9 Let $\rho$ be a simplex of the characteristic disc $\Delta$ for some thick interval $(i, j)$ (for $\left.\left(\sigma_{k}\right),\left(\tau_{k}\right)\right)$. Its characteristic image is a simplex in $X$, denoted by $\mathcal{S}(\rho)$, which is the span of the images of $\rho$ under all possible characteristic surfaces. Note that $\mathcal{S}(\rho)$ is a simplex by Lemma 9.8(iii)-(iv), and if $\rho \subset \rho^{\prime}$, then $\mathcal{S}(\rho) \subset \mathcal{S}\left(\rho^{\prime}\right)$, ie $\mathcal{S}$ respects inclusions. The characteristic image of a subcomplex of $\Delta$ is the union of the characteristic images of all its simplices. We call this assignment the characteristic mapping.

If $\bar{v}$ is a vertex in $\mathcal{S}(\Delta)$, we denote by $\mathcal{S}^{-1}(\bar{v})$ the vertex $v \in \Delta$ such that $\mathcal{S}(v) \ni \bar{v}$. We claim that this vertex is unique. Indeed, characteristic images of different layers in $\Delta$ between $v_{i} w_{i}, v_{j} w_{j}$ are disjoint since, by Remark 9.7, they lie in different layers in $X$ between $\sigma, \tau$, which are disjoint by Lemma 8.3. Moreover, by Lemma 9.8(i),(iii), we have that $S_{1}(v) \neq S_{2}\left(v^{\prime}\right)$ for any characteristic surfaces $S_{1}, S_{2}$ and any vertices $v \neq v^{\prime}$ in a common layer in $\Delta$. This justifies the claim. If $\bar{\rho}$ is a simplex in $\mathcal{S}(\Delta)$, 
we denote by $\mathcal{S}^{-1}(\bar{\rho})$ the span of the union of $\mathcal{S}^{-1}(\bar{v})$ over all $\bar{v} \in \bar{\rho}$. We have that $\mathcal{S}^{-1}(\bar{\rho})$ is a simplex, by Remark 9.7, Lemma 8.3, and Lemma 9.8(i),(iii). If $Y$ is a subcomplex of $\mathcal{S}(\Delta)$, we denote by $\mathcal{S}^{-1}(Y)$ the union of $\mathcal{S}^{-1}(\bar{\rho})$ over all $\bar{\rho} \subset Y$.

Having established the uniqueness properties of characteristic surfaces, we start to exploit the CAT( 0$)$ structure of the corresponding characteristic discs. From now on, up to the end of Section 13, unless stated otherwise, we assume that $\left(\sigma_{k}\right),\left(\tau_{k}\right)$ are the directed geodesics between $\sigma, \tau$.

Definition 9.10 Let $(i, j)$ be a thick interval and let $\Delta \subset \mathbb{E}_{\Delta}^{2}$ be its characteristic disc. We will define a sequence of simplices $\rho_{k} \in \Delta$, where $i<k<j$, which will be called the Euclidean diagonal of the characteristic disc $\Delta$.

Let $v_{k}^{\prime}, w_{k}^{\prime}$ be points (barycenters of edges) on the straight line segments $v_{k} w_{k}$ at distance $1 / 2$ from $v_{k}, w_{k}$, respectively. In particular $v_{i}^{\prime}=w_{i}^{\prime}, v_{j}^{\prime}=w_{j}^{\prime}$. Consider the closed polygonal domain $\Delta^{\prime} \subset \Delta$ enclosed by the piecewise linear loop with consecutive vertices $v_{i}^{\prime}, v_{i+1}^{\prime}, \ldots, v_{j}^{\prime}=w_{j}^{\prime}, w_{j-1}^{\prime}, \ldots, w_{i}^{\prime}=v_{i}^{\prime}$. Note that, since $\Delta^{\prime}$ is simply connected, it is $\operatorname{CAT}(0)$ with the Euclidean path metric induced from $\mathbb{E}_{\Delta}^{2}$ identified with $\mathbb{E}^{2}$. We call $\Delta^{\prime}$ a modified characteristic disc. Let $\gamma^{\prime}$ be the CAT(0) geodesic joining $v_{i}^{\prime}=w_{i}^{\prime}$ to $v_{j}^{\prime}=w_{j}^{\prime}$ in $\Delta^{\prime}$. We call $\gamma^{\prime}$ a CAT(0) diagonal of $\Delta$. For each $i<k<j$, among the vertices of $\Delta$ lying in the interior of the 1-skeleton geodesic $v_{k} w_{k}$ we find the ones nearest to $\gamma^{\prime} \cap v_{k} w_{k}$. For each $k$ this is either a single vertex or two vertices spanning an edge (if $\gamma^{\prime}$ goes through its barycenter and $v_{k}, w_{k}$ are not some of its vertices). We put $\rho_{k}$ equal to this vertex or this edge, accordingly.

At first sight it might seem strange that in the above definition we pass to $\Delta^{\prime}$ and take the geodesic $\gamma^{\prime}$ there instead of doing it in $\Delta$ itself. However, this construction allows us to exclude $v_{k}, w_{k}$ from being in $\rho_{k}$, which a careful reader will find to be a necessary condition for the arguments of the combinatorial Proposition 10.2 to be valid.

Here are some basic properties of the Euclidean diagonals.

Lemma 9.11 (i) Each pair of consecutive $\rho_{k}, \rho_{k+1}$, for $i<k<j-1$, spans a simplex.

(ii) $\rho_{i+1}, v_{i}, w_{i}$ span a simplex and $\rho_{j-1}, v_{j}, w_{j}$ span a simplex.

Proof Part (ii) is obvious, since we excluded $v_{k}, w_{k}$ from being in $\rho_{k}$. To prove (i), consider $\Delta^{\prime} \subset \Delta \subset \mathbb{E}_{\Delta}^{2}$ oriented in such a way that $v_{k} w_{k}$ are horizontal, this is possible by Lemma 9.6(ii). Moreover, Lemma 9.6(ii) yields that the boundary of $\Delta^{\prime}$ 
consists of line segments at angle $30^{\circ}$ from the vertical direction. Let $\gamma^{\prime}$ be as in Definition 9.10. It is a broken line with vertices at the boundary of $\Delta^{\prime}$.

We claim that any line segment of $\gamma^{\prime}$ is at angle less than $30^{\circ}$ from the vertical direction. First we prove that this angle is at most $30^{\circ}$. Otherwise, let $p$ be an endpoint of such a line segment. Obviously $p$ is different from the endpoints of $\gamma^{\prime}$. The interior angle at $p$ between the segment of $\gamma^{\prime}$ and any of the boundary line segments of $\Delta^{\prime}$ is less than $180^{\circ}$, which contradicts the fact that $p$ is an interior vertex of a geodesic $\gamma^{\prime}$. Thus we proved that any line segment of $\gamma^{\prime}$ is at angle at most $30^{\circ}$ from the vertical direction.

If for some line segment of $\gamma^{\prime}$ this angle equals $30^{\circ}$, then by the previous considerations the whole $\gamma^{\prime}$ is in fact a straight line at angle $30^{\circ}$ from the vertical. This implies that the defects at all vertices in $\left(v_{k}\right)_{k=i+1}^{j-1}$ or all vertices in $\left(w_{k}\right)_{k=i+1}^{j-1}$ are zero. Contradiction. We have thus proved the claim.

Now part (i) follows from the following observation, whose proof is easy and is left to the reader. Consider two consecutive horizontal lines $\alpha_{1}, \alpha_{2}$ in $\mathbb{E}_{\Delta}^{2}$. Let $\beta$ be some straight line segment joining points $p \in \alpha_{1}, r \in \alpha_{2}$ at angle less than $30^{\circ}$ from the vertical direction. Then there exist two 2-simplices $a b c, b c d$ in $\mathbb{E}_{\Delta}^{2}$ such that $a b \subset \alpha_{1}, c d \subset \alpha_{2}$ and $p \in a b, r \in c d$. Moreover, it cannot happen simultaneously that $|p a| \leq|p b|$ and $|r d| \leq|r c|$.

Thus we can finally introduce the main definition of this section.

Definition 9.12 We define a sequence of simplices $\delta_{k}$, where $0 \leq k \leq n$, which is called the Euclidean geodesic between $\sigma, \tau$, as follows. For each $k$, if the layer $k$ is thin, then we take $\delta_{k}$ to be the span of $\sigma_{k}$ and $\tau_{k}$.

If the layer $k$ is thick, consider the thick interval $(i, j)$ which contains $k$. Let $\rho_{k}$ be an appropriate simplex of the Euclidean diagonal of the characteristic disc $\Delta$ for $(i, j)$ (cf Definition 9.10). We take $\delta_{k}=\mathcal{S}\left(\rho_{k}\right)$ (cf Definition 9.9).

Remark 9.13 In the above setting, we have $\sigma_{i}=\mathcal{S}\left(v_{i}\right), \tau_{i}=\mathcal{S}\left(w_{i}\right)$, by Lemma 9.3(ii). Hence $\delta_{i}=\operatorname{span}\left\{\sigma_{i}, \tau_{i}\right\}=\mathcal{S}\left(v_{i} w_{i}\right)$.

Remark 9.14 By the symmetry of the construction, the Euclidean geodesic between $\sigma$ and $\tau$ becomes the Euclidean geodesic between $\tau$ and $\sigma$ if we take the simplices of the sequence in the opposite order.

Here is the justification for using the name "geodesic" in Definition 9.12.

Lemma 9.15 (i) For any $0 \leq k<l \leq n$ we have that $\delta_{k} \subset S_{l-k}\left(\delta_{l}\right), \delta_{l} \subset S_{l-k}\left(\delta_{k}\right)$. 
(ii) For any $0 \leq k \leq n-1$ if the layer $k$ or the layer $k+1$ is thick, then $\delta_{k}$ and $\delta_{k+1}$ span a simplex.

(iii) For any $0 \leq l<m \leq n$ such that there exists $l \leq k \leq m$ such that the layer $k$ is thick, and for any vertices $x \in \delta_{m}, y \in \delta_{l}$, we have $|x y|=m-l$.

Proof Assertion (ii) follows from Lemma 9.11(i)-(ii), Remark 9.13 and Lemma 9.8(iii)-(iv).

To prove assertion (i), say the first inclusion, observe that for any $0 \leq k<n$ we have $\operatorname{span}\left(\sigma_{k} \cup \tau_{k}\right) \subset B_{1}\left(\operatorname{span}\left(\sigma_{k+1} \cup \tau_{k+1}\right)\right)$. Hence, assertion (ii) gives already, for any $0 \leq k<l \leq n$, that $\delta_{k} \subset B_{l-k}\left(\delta_{l}\right)$. Then $\delta_{k} \subset S_{l-k}\left(\delta_{l}\right)$ follows from Remark 9.7 and Lemma 8.3(ii).

To prove part (iii), assume that $l<k<m$ (other cases are easier). Take any vertex $z \in \delta_{k}$. Then, by (i), there are vertices $x^{\prime} \in \delta_{k-1}, y^{\prime} \in \delta_{k+1}$ such that $\left|x x^{\prime}\right|=$ $(k-1)-l,\left|y y^{\prime}\right|=m-(k+1)$. By (ii) (and (i)), we have $\left|z x^{\prime}\right|=\left|z y^{\prime}\right|=1$. Hence $|x y| \leq m-l$ and by (i) we have $|x y|=m-l$, as desired.

Now we state an extra property of characteristic discs in the case where $\left(\sigma_{k}\right)$ (but $\left(\tau_{k}\right)$ not necessarily) is the directed geodesic. This property was not necessary for Definition 9.12 , but will become indispensable in the next section.

Lemma 9.16 (i) If the defect at some $v_{k}$, where $i+1<k<j-1$, equals -1 , then the defect at $v_{k+1}$ equals 1 .

(ii) The defect at $v_{i+1}$ equals 1 .

Proof (i) Proof by contradiction. Suppose the defect at some $v_{k}$, where $i+1<k<$ $j-1$, equals -1 , and the defect at $v_{k+1}$ equals 0 . Denote by $x$ the vertex next to $v_{k+1}$ on the 1 -skeleton geodesic $v_{k+1} w_{k+1}$ and by $y$ the vertex next to $v_{k}$ on the 1 -skeleton geodesic $v_{k} w_{k}$. We aim to prove that, for any characteristic surface $S$, $S(x)$ belongs to $\sigma_{k+1}$. Suppose for a moment we have already proved this. Then, since by Lemma 9.8(i) we have $\left|S(x) S\left(v_{k+2}\right)\right|=2$ and at the same time $S\left(v_{k+2}\right) \in \sigma_{k+2}$, we get a contradiction.

Now we prove that $S(x) \in \sigma_{k+1}$. By Remark 9.7 we have that $S(x)$ lies in $B_{n-k-1}(\tau)$. Hence by the definition of projection (cf Definition 2.9) it remains to prove that $S(x)$ is a neighbor of each $\bar{z} \in \sigma_{k}$. Case $\bar{z}=S(y)$ is obvious, so suppose $\bar{z} \neq S(y)$. Since, by the definition of thickness, $\left|\bar{z} S\left(w_{k}\right)\right| \leq\left|S\left(v_{k}\right) S\left(w_{k}\right)\right|$, we have by Corollary 8.7 (applied to $r_{0}=S\left(v_{k}\right), r_{1}=S(y), r_{d}=p_{d}=S\left(w_{k}\right)$ and to $p_{0}=\bar{z}$ in case of $\left|\bar{z} S\left(w_{k}\right)\right|=\left|S\left(v_{k}\right) S\left(w_{k}\right)\right|$ or to $p_{0}=S\left(v_{k}\right), p_{1}=\bar{z}$ in case of $\left|\bar{z} S\left(w_{k}\right)\right|<$ 
$\left.\left|S\left(v_{k}\right) S\left(w_{k}\right)\right|\right)$ that $|\bar{z} S(y)|=1$. Considering the loop $\bar{z} S(y) S(x) S\left(v_{k+1}\right) \bar{z}$, since $\left|S(y) S\left(v_{k+1}\right)\right|=\left|y v_{k+1}\right|=2$ (Lemma 9.8(i)), we get $|\bar{z} S(x)|=1$, as desired.

(ii) By contradiction. Denote by $x$ the vertex between $v_{i+1}$ and $w_{i+1}$ on the 1skeleton geodesic $v_{i+1} w_{i+1}$. Since $\sigma_{i}=\mathcal{S}\left(v_{i}\right)$ (see Remark 9.13), we have by Remark 9.7 and Lemma 9.8(iii) that $S(x)$ belongs to $\sigma_{i+1}$. By Lemma 9.8(i) we have $\left|S(x) S\left(v_{i+2}\right)\right|=2$. At the same time $S\left(v_{i+2}\right) \in \sigma_{i+2}$, contradiction.

We will repeat some steps of this proof later on in the proof of Lemma 10.3. We decided, for clarity, not to intertwine these two proofs.

As a consequence of Lemma 9.16, we get the following lemma, whose proof, similar to the proof of Lemma 9.11, we omit. Here we assume that both $\left(\sigma_{k}\right),\left(\tau_{k}\right)$ are directed geodesics.

Lemma 9.17 If $j-i>2$ then the CAT(0) diagonal $\gamma^{\prime}$ in $\Delta$ crosses each line orthogonal to the layers transversally.

\section{Directed geodesics between simplices of Euclidean geode- sics}

In this section we start to prove a weak version of Theorem B, which concerns one of the main properties of Euclidean geodesics. Roughly speaking, the theorem says that pieces of Euclidean geodesics are coarsely also Euclidean geodesics.

We keep the notation from the previous section. The simplices $\left(\sigma_{k}\right),\left(\tau_{k}\right)$ are in this section the directed geodesics between $\sigma, \tau$.

Theorem 10.1 (Weak version of Theorem B) Let $\sigma, \tau$ be simplices of a systolic complex $X$, such that for some natural $n$ we have $\sigma \subset S_{n}(\tau), \tau \subset S_{n}(\sigma)$ (as required in the definition of the Euclidean geodesic). Let $\left(\delta_{k}\right)_{k=0}^{n}$ be the Euclidean geodesic between $\sigma$ and $\tau$. Take some $0 \leq l<m \leq n$ and consider the simplices $\tilde{\delta}_{l}=$ $\delta_{l}, \tilde{\delta}_{l+1}, \ldots, \tilde{\delta}_{m}=\delta_{m}$ of the Euclidean geodesic between $\delta_{l}$ and $\delta_{m}$ (we can define it by Lemma 9.15(i)). Then for each $l \leq k \leq m$ we have $\left|\delta_{k}, \widetilde{\delta}_{k}\right| \leq 3$.

The proof of Theorem 10.1 splits into two steps. The first step is to prove that directed geodesics between $\delta_{l}$ and $\delta_{m}$ stay close to the union of characteristic images of all characteristic discs (for $\left(\sigma_{k}\right),\left(\tau_{k}\right)$ ). This is the content of Proposition 10.2, whose proof occupies the rest of this section. 
The second step is to check that characteristic images for the directed geodesics between $\delta_{l}$ and $\delta_{m}$ also stay close to the union of characteristic images for $\left(\sigma_{k}\right),\left(\tau_{k}\right)$. Properties of layers actually imply that characteristic discs of the former are embedded into characteristic discs of the latter, modulo small neighborhood of the boundary. So everything boils down to the fact that Theorem 10.1 is valid for CAT(0) subspaces of the Euclidean plane. We carry out this program in the next section. We also indicate there an argument, how to promote Theorem 10.1 to Theorem B, with a reasonable constant $C$.

A complete alternative proof of Theorem $\mathrm{B}$, with a worse constant $C$, is obtained as a consequence of Proposition 12.1. We present it at the end of Section 12. We advise the reader to have a look at the proof of Theorem 10.1 via Proposition 10.2. This proof is straightforward and allows us to introduce gradually some concepts needed later. However, to save time, one can skip the remaining part of Section 10, go over the definitions in Section 11 and then go directly to Section 12.

For each thick layer $l \leq k \leq m$ contained in a thick interval $(i, j)$ (for $\left(\sigma_{t}\right),\left(\tau_{t}\right)$; from now on we often skip "for $\left(\sigma_{t}\right),\left(\tau_{t}\right)$ "), denote by $\alpha_{k}$ the appropriate simplex (in the corresponding characteristic disc $\Delta$ ) of the directed geodesic from $\rho_{l}$, if $i<l$, or $v_{i}$ otherwise, to $\rho_{m}$, if $m<j$, or $v_{j}$ otherwise. The simplices $\left(\widetilde{\sigma}_{k}\right)_{k=l}^{m}$ of the directed geodesic from $\delta_{l}$ to $\delta_{m}$ satisfy the following.

Proposition 10.2 Let $l \leq k \leq m$.

(i) If the layer $k$ is thin, then $\widetilde{\sigma}_{k}$ contains or is contained in $\sigma_{k}$.

(ii) If the layer $k$ is thick, then $\widetilde{\sigma}_{k}$ contains or is contained in $\mathcal{S}\left(\alpha_{k}\right)$.

Before we give the proof of Proposition 10.2, we need to establish some necessary lemmas. The first one describes the position of $\sigma_{k}$ with respect to the characteristic image. Like in Lemma 9.16, here $\left(\tau_{k}\right)$ does not need to be the directed geodesic.

Lemma 10.3 For a thick layer $k$ let $x_{k}$ be the vertex, which is the neighbor of $v_{k}$ on the 1-skeleton geodesic $v_{k} w_{k}$ in the characteristic disc for the thick interval containing $k$. If the defect at $v_{k}$ equals 1 , then $\sigma_{k}=\mathcal{S}\left(v_{k} x_{k}\right)$. Otherwise $\sigma_{k}=\mathcal{S}\left(v_{k}\right)$.

Proof First of all $\sigma_{k} \subset \mathcal{S}\left(v_{k} x_{k}\right)$ follows from the definition of thickness and Proposition 7.6 (one could also verify this by hand, similarly like in the proofs of Lemma 9.8(iii) and Lemma 9.16(i)). Suppose that the defect at $v_{k}$ is $\neq 1$. Hence $\left|v_{k-1} x_{k}\right|=2$, by Lemma 9.16(i)-(ii). The inclusion $\mathcal{S}\left(v_{k}\right) \subset \sigma_{k}$ is obvious and the converse inclusion follows from $\sigma_{k} \subset \mathcal{S}\left(v_{k} x_{k}\right)$ and from Lemma 9.8(i). 
Now suppose the defect at $v_{k}$ equals 1 . If the layer $k-1$ is thick, then the defect at $v_{k-1}$ is $\neq 1$ and we apply what we have just proved to get $\mathcal{S}\left(v_{k-1}\right)=\sigma_{k-1}$. If the layer $k-1$ is thin we get immediately that $\mathcal{S}\left(v_{k-1}\right)=\sigma_{k-1}$ (Remark 9.13). In both cases using Remark 9.7, Lemma 9.8(iii), and the definition of projection we get $\mathcal{S}\left(v_{k} x_{k}\right) \subset \sigma_{k}$, as desired.

As a corollary we get the following technical lemma.

Lemma 10.4 Suppose $k<m$ do not satisfy $i \leq k<m<j$ for any thick interval $(i, j)$ or if they violate this then $\left|v_{k+1}, \rho_{m}\right|=m-(k+1)$. Then the projection of $\sigma_{k}$ onto $B_{m-(k+1)}\left(\delta_{m}\right)$ equals $\sigma_{k+1}$.

Proof To justify speaking about the projection of $\sigma_{k}$ onto $B_{m-(k+1)}\left(\delta_{m}\right)$ we must show that $\sigma_{k} \subset S_{m-k}\left(\delta_{m}\right)$. The simplex $\sigma_{k}$ is outside $B_{m-k-1}\left(\delta_{m}\right)$ by Remark 9.7 and Lemma 8.3(ii). Thus we only need to check that $\sigma_{k} \subset B_{m-k}\left(\delta_{m}\right)$.

To verify this, we prove that $\sigma_{k+1} \subset B_{m-(k+1)}\left(\delta_{m}\right)$. If the layer $k+1$ is thin then this follows from Lemma 9.15(i). If the layer $k+1$ is thick, then denote by $(i, j)$ the thick interval containing $k+1$. By Lemma 10.3 we have $\sigma_{k+1} \subset \mathcal{S}\left(v_{k+1} x_{k+1}\right)$ $\left(x_{k+1}\right.$ as in Lemma 10.3). Thus it is enough to establish the inclusion $\mathcal{S}\left(v_{k+1} x_{k+1}\right) \subset$ $B_{m-(k+1)}(\delta)$. If $m<j$, then this follows from our hypothesis. If $j \leq m$, then from Remark 9.13 and Lemma 9.15(i) we have

$$
\begin{aligned}
\mathcal{S}\left(v_{k+1} x_{k+1}\right) \subset \mathcal{S}\left(B_{j-(k+1)}\left(v_{j}\right)\right) & \subset B_{j-(k+1)}\left(\mathcal{S}\left(v_{j}\right)\right) \\
& \subset B_{j-(k+1)}\left(\delta_{j}\right) \subset B_{m-(k+1)}\left(\delta_{m}\right),
\end{aligned}
$$

as desired.

Hence the projection of $\sigma_{k}$ onto $B_{m-(k+1)}\left(\delta_{m}\right)$ is defined. Denote it by $\pi$. Since $B_{m-(k+1)}\left(\delta_{m}\right) \subset B_{n-(k+1)}(\tau)$, we have $\pi \subset \sigma_{k+1}$. For the converse inclusion we need $\sigma_{k+1} \subset B_{m-(k+1)}\left(\delta_{m}\right)$, which we have just proved.

The next lemma is valid for any $\left(\sigma_{k}\right),\left(\tau_{k}\right)$, not necessarily directed geodesics.

Lemma 10.5 Let $e$ be an edge in the layer $k$ of $\Delta$ (between $v_{i} w_{i}, v_{j} w_{j}$ ), such that $e$ has three neighboring vertices in the layer $k+1$. Let $\bar{x}$ be a vertex in the residue (defined before Lemma 2.8) of $S(e)$ (for some characteristic surface $S$ ) in the layer $k+1$ between $\sigma, \tau$ in $X$. Then $\bar{x} \in \mathcal{S}(x)$, where $x$ is the vertex in the layer $k+1$ of $\Delta$ in the residue of $e$. 
Proof Denote by $y_{1}, y_{2}$ the neighbors of $e$ in the layer $k+1$ of $\Delta$ different from $x$, and let $\bar{y}_{1}=S\left(y_{1}\right), \bar{y}_{2}=S\left(y_{2}\right)$. We claim that $\bar{y}_{1}, \bar{y}_{2}$ are neighbors of $\bar{x}$. Indeed, let $z_{1}$ be the vertex in $e$, which is a neighbor of $y_{1}$. Let $\bar{z}_{1}=S\left(z_{1}\right) \subset S(e)$. Observe that both $\bar{y}_{1}, \bar{x}$ lie in the projection of $\bar{z}_{1}$ onto $B_{n-(k+1)}(\tau)$ (by Remark 9.7), hence, by Lemma 2.8 , they are neighbors, as desired. Analogously, $\bar{y}_{2}, \bar{x}$ are neighbors. Thus, by the easy case of Proposition 7.6, we have that $\bar{x} \in \mathcal{S}(x)$, as required.

The following lemma describes the behavior of the simplices $\alpha_{k}$ appearing in the statement of Proposition 10.2. The proof of Lemma 10.6 requires Lemma 9.16(i)-(ii), apart from this it is straightforward and we skip it. For the same reason we will usually not invoke it in the proof of Proposition 10.2.

Lemma 10.6 Let $\Delta$ be a characteristic disc for some thick interval $(i, j)$. Suppose that for some $i \leq l<m \leq j$ we have simplices $\alpha, \alpha^{\prime}$ in the layers $l, m$ respectively between $v_{i} w_{i}, v_{j} w_{j}$ in $\Delta$. Suppose that $\alpha \subset S_{m-l}\left(\alpha^{\prime}\right)$ and $\alpha^{\prime} \subset S_{m-l}(\alpha)$. Moreover, assume that $\alpha$ is an interior vertex of $\Delta$ or an edge disjoint with the boundary or $\alpha=v_{i}$. Assume that $\alpha^{\prime}$ is an interior vertex or an edge disjoint with boundary or $\alpha^{\prime}=v_{j}$. Let $\left(\alpha_{k}\right)_{k=l}^{m}$ be the directed geodesic in $\Delta$ joining $\alpha$ to $\alpha^{\prime}$ (in particular $\alpha_{l}=\alpha, \alpha_{m} \subset \alpha^{\prime}$ ). Then:

(i) If $\alpha_{k}$ is an edge, then $\alpha_{k+1}$ is the unique vertex, which is in the residue of $\alpha_{k}$ in the layer $k+1$.

(ii) If $\alpha_{k}=v_{k}$ and the defect at $v_{k}$ equals 0 , then $\alpha_{k+1}=v_{k+1}$.

(iii) If $\alpha_{k}$ is a vertex with two neighbors in the layer $k+1$, both at distance $m-(k+1)$ from $\alpha^{\prime}$, then $\alpha_{k+1}$ is an edge spanned by these two vertices.

(iv) If $\alpha_{k}$ is a vertex with two neighbors in the layer $k+1$, but only one of them at distance $m-(k+1)$ from $\alpha^{\prime}$, then $\alpha_{k+1}$ is this special vertex.

(v) Moreover, $\alpha_{k}$ never equals $w_{k}$. If $\alpha_{k}$ is an edge containing $w_{k}$ then the defect at $w_{k}$ is -1 . If $\alpha_{k}=v_{k}$, then the defect at $v_{k}$ is not equal to 1 , except possibly for the cases $k=i, j$.

Now we are ready for the following.

Proof of Proposition 10.2 We prove by induction on $k$, for $l \leq k \leq m$, the following statement, which, by Lemma 10.6 and Lemma 10.3, implies the proposition.

Induction hypothesis (1) If the layer $k$ is thick and $\alpha_{k}$ is an edge disjoint with the boundary or meeting the boundary at a vertex of defect $\neq 1$, then $\widetilde{\sigma}_{k}$ contains $\mathcal{S}\left(\alpha_{k}\right)$. 
(2) If the layer $k$ is thick and $\alpha_{k}$ is a nonboundary vertex, then $\tilde{\sigma}_{k}$ is contained $\mathcal{S}\left(\alpha_{k}\right)$.

(3) If the layer $k$ is thick and $\alpha_{k}$ is a boundary vertex or an edge intersecting the boundary at a vertex of defect 1 , or the layer $k$ is thin, then $\widetilde{\sigma}_{k}$ contains or is contained in $\sigma_{k}$.

For $k=l$ the hypothesis is obvious. Suppose it is already proved for some $l \leq k \leq m-1$. We would like to prove it for $k+1$. First suppose that the layer $k$ is thick and $\alpha_{k}$ is an edge disjoint with the boundary or meeting the boundary at a vertex of defect $\neq 1$ (case (1)). Then $\alpha_{k+1}$ is a vertex. If it is a boundary vertex, then $v_{k} \in \alpha_{k}$. By the induction hypothesis, since the defect at $v_{k}$ is not $1, \mathcal{S}\left(\alpha_{k}\right) \subset \widetilde{\sigma}_{k}$, moreover, by Lemma 10.3 we have $\sigma_{k} \subset \mathcal{S}\left(\alpha_{k}\right)$, hence $\sigma_{k} \subset \widetilde{\sigma}_{k}$. Hence, by Lemma 2.10, $\widetilde{\sigma}_{k+1}$ is contained in the projection of $\sigma_{k}$ onto $B_{m-(k+1)}\left(\delta_{m}\right)$, which in this case equals $\sigma_{k+1}$ by Lemma 10.4. Thus $\widetilde{\sigma}_{k+1} \subset \sigma_{k+1}$, as desired.

Now, still assuming that the layer $k$ is thick and that $\alpha_{k}$ is an edge disjoint with the boundary or meeting the boundary at a vertex of defect $\neq 1$, suppose that $\alpha_{k+1}$ is not a boundary vertex. Let $\bar{x}$ be any vertex in $\widetilde{\sigma}_{k+1}$. Our goal is to prove that $\bar{x} \in \mathcal{S}\left(\alpha_{k+1}\right)$. By induction hypothesis we know that $\mathcal{S}\left(\alpha_{k}\right) \subset \widetilde{\sigma}_{k}$. Since $\bar{x}$ lies in the layer $k+1$ between $\sigma, \tau$, by Remark 9.7, we can apply Lemma 10.5 with $e=\alpha_{k}$. Hence we get $\bar{x} \in \mathcal{S}\left(\alpha_{k+1}\right)$, as desired.

Thus we have completed the induction step in case (1), ie for the layer $k$ thick and $\alpha_{k}$ an edge disjoint with the boundary or meeting the boundary at a vertex of defect $\neq 1$.

Now suppose that the layer $k$ is thick and $\alpha_{k}$ is a nonboundary vertex (case (2)). Then it has two neighbors in the layer $k+1$ of $\Delta$, suppose first that both of them are at distance $m-(k+1)$ from $\rho_{m}$ (we put $\rho_{m}=v_{j}$ if $m \geq j$ ). Then $\alpha_{k+1}$ is the edge spanned by those two vertices. If it intersects the boundary, the defect at the boundary vertex is not 1. Thus we must show that $\widetilde{\sigma}_{k+1}$ contains $\mathcal{S}\left(\alpha_{k+1}\right)$. But by induction hypothesis we know that $\widetilde{\sigma}_{k}$ is contained in $\mathcal{S}\left(\alpha_{k}\right)$. Thus, by Lemma 2.10, it is enough to observe that $\mathcal{S}\left(\alpha_{k+1}\right) \subset B_{m-(k+1)}\left(\delta_{m}\right)$. This follows from $\alpha_{k+1} \subset B_{m-(k+1)}\left(\rho_{m}\right)$.

If one of the two neighbors of $\alpha_{k}$ in the layer $k+1$ is not at distance $m-(k+1)$ from $\rho_{m}$, then $\alpha_{k+1}$ is the second neighbor, it is a nonboundary vertex (unless $k+1=j$, which will be considered in a moment) and $m<j$. Thus we must show that $\widetilde{\sigma}_{k+1}$ is contained in $\mathcal{S}\left(\alpha_{k+1}\right)$. Let $\bar{z}$ be a vertex in $\tilde{\sigma}_{k+1}$. Then $\bar{z}$ lies on a $1-$ skeleton geodesic $\bar{\gamma}$ of length $m-k$ from some vertex of $\widetilde{\sigma}_{k} \subset \mathcal{S}\left(\alpha_{k}\right)$ to some vertex $\bar{x} \in \delta_{m}=\mathcal{S}\left(\rho_{m}\right)$. We claim that if $\rho_{m}$ is an edge, then the vertex $x=\mathcal{S}^{-1}(\bar{x}) \in \Delta$ is the vertex closer to $v_{m}$ then the other vertex of $\rho_{m}$. Indeed, let $y \in \rho_{m}$ be the vertex closer to $w_{m}$. Since $\left|\alpha_{k} y\right|>m-k$ and this distance is realized by a neat geodesic, hence by Lemma 9.8(i) 
we have $\left|\mathcal{S}\left(\alpha_{k}\right), \mathcal{S}(y)\right|>m-k$. This proves the claim. Thus we can apply Proposition 7.6 to $\gamma=\alpha_{k} x$ and $\bar{z} \in \bar{\gamma}$, and get $\bar{z} \in \mathcal{S}\left(\alpha_{k+1}\right)$, as desired.

Now we come back to the case $k+1=j$ and $\alpha_{k}$ a nonboundary vertex. By induction hypothesis we have $\tilde{\sigma}_{k} \subset \mathcal{S}\left(\alpha_{k}\right)$. By Lemma 9.15(i) we have that $\sigma_{k+1}=\mathcal{S}\left(v_{k+1}\right)$ (Remark 9.13) lies in $B_{m-(k+1)}\left(\delta_{m}\right)$. Hence, by Lemma 2.10, we have that $\tilde{\sigma}_{k+1}$ contains $\sigma_{k+1}$, as desired.

Thus we have completed the induction step in case (2), ie for the layer $k$ thick and $\alpha_{k}$ a nonboundary vertex.

Now consider the case that the layer $k$ is thick and $\alpha_{k}$ is a boundary vertex of defect -1 or the layer $k$ is thin, but the layer $k+1$ is thick (in this case put $i=k$ ). In both cases $\alpha_{k}=v_{k}$. If the hypothesis of Lemma 10.4 are not satisfied, then we can finish as in the previous case (no matter what is the direction of the inclusion given by the induction hypothesis) getting $\widetilde{\sigma}_{k+1} \subset \mathcal{S}\left(\alpha_{k+1}\right)$. Otherwise, $\alpha_{k+1}$ is the edge spanned by two neighbors of $v_{k}$ in the layer $k+1$. By Lemma 9.16(i)-(ii) the defect at $v_{k+1}$ equals 1 . Hence we want to prove that $\tilde{\sigma}_{k+1}$ either contains or is contained in $\sigma_{k+1}$. We know, by the induction hypothesis, that $\widetilde{\sigma}_{k}$ contains or is contained in $\sigma_{k}$, hence it is enough to use Lemma 2.10 and Lemma 10.4.

Now assume that either the layer $k$ is thick and $\alpha_{k}$ is a boundary vertex of defect 0 or an edge intersecting the boundary at a vertex of defect 1 , or the layer $k$ is thin and the layer $k+1$ is also thin. Similarly as before, we have that $\widetilde{\sigma}_{k}$ contains or is contained in $\sigma_{k}$ and we want to prove that $\widetilde{\sigma}_{k+1}$ contains or is contained in $\sigma_{k+1}$. This follows from Lemma 2.10 and Lemma 10.4.

Thus we have exhausted all the possibilities for case (3) and completed the induction step.

\section{Euclidean geodesics between simplices of Euclidean geo- desics}

In this section we complete the proof of Theorem 10.1. Its first ingredient is Proposition 10.2, proved in Section 10. The second ingredient is easy 2-dimensional Euclidean geometry, which we present as a series of lemmas in this section. Throughout the section, we will be treating characteristic discs simultaneously as simplicial complexes and $\mathrm{CAT}(0)$ metric spaces.

We start with extending in various ways the notion of a characteristic disc and surface. 
Definition 11.1 A generalized characteristic disc $\Delta$ for an interval $(i, j)$, where $i<j$, is a closed CAT(0) (ie simply connected) subspace of $\mathbb{E}^{2}$ with the following properties. Its boundary is a piecewise linear loop with vertices $v_{i}, \ldots, v_{j}, w_{j}, \ldots, w_{i}, v_{i}$ (possibly $v_{k}=w_{k}$ ), such that for $i \leq k \leq j$ the straight line segments (or points) $v_{k} w_{k}$ are contained in consecutive parallel lines at distance $\sqrt{3} / 2$. We also require, if $\mathbb{E}^{2}$ is oriented so that $v_{k} w_{k}$ are horizontal, that $v_{k}$ lies to the left of $w_{k}$, or $v_{k}=w_{k}$.

A restriction of a generalized characteristic disc to the interval $(l, m)$, where $i \leq l<$ $m \leq j$, is the generalized characteristic disc enclosed by the loop $v_{l} \cdots v_{m} w_{m} \cdots w_{l} v_{l}$. We denote it by $\left.\Delta\right|_{l} ^{m}$. If a generalized characteristic disc comes from equipping a systolic 2-complex with the standard piecewise Euclidean metric, then we call it a simplicial generalized characteristic disc.

Remark 11.2 Characteristic discs (resp. modified characteristic discs, cf Definition 9.10) with the standard piecewise Euclidean metric are simplicial generalized characteristic discs (resp. generalized characteristic discs).

Definition 11.3 Suppose that we have simplices $\left(\sigma_{k}\right),\left(\tau_{k}\right)$ in the layer $k$ between $\sigma, \tau$ (not necessarily the simplices of the directed geodesics) defined (only) for $0 \leq$ $i \leq k \leq j \leq n$, where $i<j$, such that for $i \leq k<j$ we have that $\sigma_{k}, \sigma_{k+1}$ span a simplex and $\tau_{k}, \tau_{k+1}$ span a simplex. Suppose that for $i \leq k \leq j$ the maximal distance between vertices in $\sigma_{k}$ and in $\tau_{k}$ is at least 2 . Then we define a partial characteristic disc and a partial characteristic surface in the following way.

We extend $\left(\sigma_{k}\right),\left(\tau_{k}\right)$ to all $0 \leq k \leq n$ so that $\sigma_{k}, \sigma_{k+1}$ and $\tau_{k}, \tau_{k+1}$ span simplices for $0 \leq k<n$, and $\sigma_{0}, \tau_{0} \subset \sigma, \sigma_{n}, \tau_{n} \subset \tau$. (This is possible, since, for example, we may issue directed geodesics from $\sigma_{i}, \tau_{i}$ to $\sigma$ and from $\sigma_{j}, \tau_{j}$ to $\tau$.) Obviously, $\sigma_{k}, \tau_{k}$ lie in the layer $k$ between $\sigma, \tau$ for all $0 \leq k \leq n$. Let $\left(i_{\text {ext }}, j_{\text {ext }}\right)$ be the thick interval for extended $\left(\sigma_{k}\right),\left(\tau_{k}\right)$ containing $(i, j)$. Let $S: \Delta \rightarrow X$ be a characteristic surface for $\left(i_{\text {ext }}, j_{\text {ext }}\right)$. Then we call $\Delta_{\text {res }}=\left.\Delta\right|_{i} ^{j}$ a partial characteristic disc (which is a simplicial generalized characteristic disc) and $S_{\text {res }}=\left.S\right|_{\Delta_{\text {res }}}$ a partial characteristic surface.

Caution A characteristic surface $S: \Delta \rightarrow X$, where $\Delta$ is a characteristic disc for a thick interval $(i, j)$ for $\left(\sigma_{k}\right)_{k=0}^{n},\left(\tau_{k}\right)_{k=0}^{n}$ (as in Definition 9.4) is not a partial characteristic surface for $\left(\sigma_{k}\right)_{k=i}^{j},\left(\tau_{k}\right)_{k=i}^{j}$. This is because the layers $i, j$ are thin. But if $i+1<j-1$, then already $S$ restricted to $\left.\Delta\right|_{i+1} ^{j-1}$ is a partial characteristic surface.

Next we show that partial characteristic surfaces satisfy most of the properties of characteristic surfaces. Fix an interval $(i, j)$ and simplices $\left(\sigma_{k}\right)_{k=i}^{j},\left(\tau_{k}\right)_{k=i}^{j}$ as in Definition 11.3. Let $S_{\text {res }}:\left.\Delta\right|_{\text {res }} \rightarrow X$ be a partial characteristic surface, as above. 
Lemma 11.4 (i) $\Delta_{\text {res }}$ (and thus $S_{\text {res }}$ ) is flat.

(ii) If we embed $\Delta_{\text {res }} \subset \mathbb{E}_{\Delta}^{2}$, then $v_{i} w_{i}$ and $v_{j} w_{j}$ are parallel and the consecutive layers between them are contained in consecutive straight lines parallel to $v_{i} w_{i}$ and $v_{j} w_{j}$.

(iii) $S_{\text {res }}$ is an isometric embedding on 1-skeleton of a subcomplex spanned by any pair of consecutive layers between $v_{i} w_{i}$ and $v_{j} w_{j}$ in $\Delta_{\text {res }}$.

(iv) $\Delta_{\text {res }} \subset \mathbb{E}_{\Delta}^{2}$ does not depend on the choice of $\sigma_{k}, \tau_{k}$ for $k<i$ and $k>j$, the choice of $s_{k}, t_{k}$ for $0 \leq k \leq n$, and the choice of $S$.

If we have two partial characteristic surfaces $S_{1}: \Delta_{1} \rightarrow X, S_{2}: \Delta_{2} \rightarrow X$, then after identifying partial characteristic discs $\Delta_{1}=\Delta_{2}$ (which is possible by (ii)) we have that

(v) for any vertices $x, y \in \Delta_{1}=\Delta_{2}$ at distance $1, S_{1}(x)$ and $S_{2}(y)$ are also at distance 1,

(vi) for any vertex $x \in \Delta_{1}=\Delta_{2}, S_{1}(x)$ and $S_{2}(x)$ are at distance at most 1 ,

(vii) $S\left(v_{k} w_{k}\right)$ lies in the layer $k$ between $\sigma$ and $\tau$.

Proof Assertions (i) and (ii) follow immediately from Lemma 9.6(i)-(ii). Assertion (iii) follows from Lemma 9.8(i). To prove (iv) notice that $\Delta_{\text {res }}=\left.\Delta\right|_{i} ^{j}$ is determined by the distances $\left|s_{k} t_{k}\right|$ for $i \leq k \leq j$ and $\left|s_{k} t_{k+1}\right|$ for $i \leq k<j$, by (iii). Hence, if we fix $s_{k}$ and $t_{k}$ for $i \leq k \leq j$, then $\Delta_{\text {res }}$ does not depend on the extension of $\left(\sigma_{k}\right)_{k=i}^{j},\left(\tau_{k}\right)_{k=i}^{j}$. On the other hand, if we fix such an extension, then $\left|s_{k} t_{k}\right|,\left|s_{k} t_{k+1}\right|$ do not depend on the choice of $s_{k}, t_{k}$, by Lemma 9.8(ii).

It is a bit awkward to try to obtain assertion (v) as a consequence of Lemma 9.8(iii). Let us say, instead, that assertion (v) follows immediately from the proof of Lemma 9.8(iii). Similarly, assertion (vi) follows from the proof of Lemma 9.8(iv).

Assertion (vii) follows directly from Remark 9.7.

Definition 11.5 We define the partial characteristic image $\mathcal{S}(\subset)$ of a simplex $\rho$ in the partial characteristic disc as the span of $S(\rho)$ over all partial characteristic surfaces $S$. By Lemma 11.4(v)-(vi), $\mathcal{S}(\subset)$ is a simplex. We call this assignment the partial characteristic mapping. Like in Definition 9.9 we can consider also the assignment $\mathcal{S}^{-1}$.

Definition 11.6 Let $\Delta$ be a generalized characteristic disc and $\gamma, \gamma^{\prime}$ be two paths connecting some points on $v_{i} w_{i}$ to points on $v_{j} w_{j}$ such that intersections of $\gamma, \gamma^{\prime}$ with $v_{k} w_{k}$ are unique for each $i \leq k \leq j$. We say that $\gamma, \gamma^{\prime}$ are $d$-close if they intersect $v_{k} w_{k}$ in points at distance at most $d$ for each $i \leq k \leq j$. 
The following lemma describes the possible displacements of CAT(0) geodesics in characteristic discs when perturbing the boundary and the endpoints.

Lemma 11.7 Let $\Delta^{\prime} \subset \Delta$ be two generalized characteristic discs for $(i, j)$ such that for each $i \leq k \leq j$ we have $v_{k}^{\prime} w_{k}^{\prime} \subset v_{k} w_{k}$ (and the order is $v_{k} v_{k}^{\prime} w_{k}^{\prime} w_{k}$ ) and $\left|v_{k} v_{k}^{\prime}\right| \leq d,\left|w_{k} w_{k}^{\prime}\right| \leq d$. Then for any points $x \in v_{i} w_{i}, y \in v_{j} w_{j}, x^{\prime} \in v_{i}^{\prime} w_{i}^{\prime}, y^{\prime} \in v_{j}^{\prime} w_{j}^{\prime}$ such that $\left|x x^{\prime}\right| \leq d,\left|y y^{\prime}\right| \leq d$, the CAT(0) geodesics from $x$ to $y$ in $\Delta$ and from $x^{\prime}$ to $y^{\prime}$ in $\Delta^{\prime}$ are $d$-close in $\Delta$.

Proof Denote by $\gamma, \gamma^{\prime}$ the geodesics from $x$ to $y$ in $\Delta$ and from $x^{\prime}$ to $y^{\prime}$ in $\Delta^{\prime}$ respectively. Denote by $N_{d}(\gamma)$ the set of points in $\Delta$ at distance $\leq d$ from $\gamma$ in the direction parallel to $v_{k} w_{k}$ (ie the intersection with $\Delta$ of the union of translates of $\gamma$ by a distance at most $d$ in the direction parallel to $v_{k} w_{k}$ ), and by $N_{d}^{\prime}(\gamma)$ the intersection $N_{d}(\gamma) \cap \Delta^{\prime}$.

Observe that $N_{d}^{\prime}(\gamma)$ is connected, since for each $k$ the set $v_{k}^{\prime} w_{k}^{\prime} \cap N_{d}^{\prime}(\gamma)$ is nonempty and the intersection of $N_{d}^{\prime}(\gamma)$ with each of the parallelograms $v_{k}^{\prime} w_{k}^{\prime} w_{k+1}^{\prime} v_{k+1}^{\prime}$ is an intersection of two parallelograms, hence convex and connected. We claim that $N_{d}(\gamma)$ is convex in $\Delta$. To establish this, we need to study the interior angle at vertices of $\partial N_{d}(\gamma)$ outside $\partial \Delta$. The only possibility for angle greater than $180^{\circ}$ is at the horizontal translates of break points of $\gamma$. But since $\gamma$ is a CAT( 0$)$ geodesic, then each of its break points lies on the boundary of $\Delta$, and the translate, for which possibly the angle is greater than $180^{\circ}$, lies outside $\Delta$. Thus the claim follows. Hence (by connectedness) $N_{d}^{\prime}(\gamma)$ is convex in $\Delta^{\prime}$. Thus $\gamma^{\prime} \subset N_{d}^{\prime}(\gamma)$ and we are done.

Let us prepare the setting for the next lemma. It will help us deal with the data given by Proposition 10.2, which is, roughly speaking, a pair of surfaces spanned on nearby pairs of geodesics. To be more precise, let $\widehat{\sigma}_{k}, \widehat{\tau}_{k}, \widetilde{\sigma}_{k}, \widetilde{\tau}_{k}$ be simplices in the layers $i \leq k \leq j$ between $\sigma, \tau$ satisfying conditions of Definition 11.3. Moreover, assume that for each $i \leq k \leq j$ we have that $\widehat{\sigma}_{k} \subset \widetilde{\sigma}_{k}$ or $\widetilde{\sigma}_{k} \subset \hat{\sigma}_{k}$, and $\hat{\tau}_{k} \subset \widetilde{\tau}_{k}$ or $\widetilde{\tau}_{k} \subset \hat{\tau}_{k}$. Let $\widehat{\Delta}, \tilde{\Delta}$ be associated partial characteristic discs, unique by Lemma 11.4(iv). Denote the boundary vertices of $\widehat{\Delta}$ (resp. $\widetilde{\Delta}$ ) by $\widehat{v}_{k}, \widehat{w}_{k}$ (resp. $\widetilde{v}_{k}, \widetilde{w}_{k}$ ), its characteristic mapping by $\hat{\mathcal{S}}(\operatorname{resp} . \tilde{\mathcal{S}})$.

Lemma 11.8 There exists a simplicial generalized characteristic disc $\bar{\Delta}$ for $(i, j)$ and embeddings (thought of as inclusions, for simplicity) $\bar{\Delta} \subset \widehat{\Delta}, \bar{\Delta} \subset \tilde{\Delta}$ such that the distances $\left|\bar{v}_{k} \widehat{v}_{k}\right|,\left|\bar{w}_{k} \widehat{w}_{k}\right|$ in $\widehat{\Delta}$ and the distances $\left|\bar{v}_{k} \widetilde{v}_{k}\right|,\left|\bar{w}_{k} \widetilde{w}_{k}\right|$ in $\widetilde{\Delta}$ are all $\leq 1$ for $i \leq k \leq j$. Moreover, $\left|\bar{v}_{k} \bar{w}_{k}\right| \geq 1$ for $i \leq k \leq j$. 
Proof For each $i \leq k \leq j$, let $\sigma_{k}^{\max }$ be the greater among $\hat{\sigma}_{k}, \widetilde{\sigma}_{k}$ and let $\sigma_{k}^{\min }$ be the smaller, let $\tau_{k}^{\max }$ be the greater among $\hat{\tau}_{k}, \tilde{\tau}_{k}$ and let $\tau_{k}^{\min }$ be the smaller. Pick vertices $x_{k} \in \sigma_{k}^{\max }, y_{k} \in \tau_{k}^{\max }$ so that the distance $\left|x_{k} y_{k}\right|$ is maximal. If possible, choose them from $\sigma_{k}^{\min }, \tau_{k}^{\min }$ (if it is possible for $x_{k}, y_{k}$ independently, then it is possible for both of them at the same time, by Lemma 9.5). Pick a 1-skeleton geodesic $\phi_{k}$ connecting $x_{k}$ to $y_{k}$ intersecting $\sigma_{k}^{\min }, \tau_{k}^{\min }$ (this is possible by Corollary 8.7). If $x_{k} \in \sigma_{k}^{\min }$, then put $\bar{s}_{k}=x_{k}$, otherwise let $\bar{s}_{k}$ be the neighbor of $x_{k}$ on $\phi_{k}$. Analogously, if $y_{k} \in \tau_{k}^{\min }$, then put $\bar{t}_{k}=y_{k}$, otherwise let $\bar{t}_{k}$ be the neighbor vertex of $y_{k}$ on $\phi_{k}$. Thus $\bar{s}_{k} \in \sigma_{k}^{\min }, \bar{t}_{k} \in \tau_{k}^{\min }$. Let $\bar{\Delta}$ be the partial characteristic disc for $\left(\bar{s}_{k}\right),\left(\bar{t}_{k}\right)$ for $i \leq k \leq j$. Denote its boundary vertices by $\bar{v}_{k}, \bar{w}_{k}$.

The embedding, say $\bar{\Delta} \subset \widehat{\Delta}$, is defined as follows. By Proposition 7.6 there exists a characteristic surface $\bar{S}: \bar{\Delta} \rightarrow X$ such that $\bar{S}\left(\bar{v}_{k} \bar{w}_{k}\right)=\bar{s}_{k} \bar{t}_{k} \subset \phi_{k}$. Moreover, again by Proposition 7.6, the subgeodesic $\bar{s}_{k} \bar{t}_{k}$ of $\phi_{k}$ lies in $\widehat{\mathcal{S}}(\widehat{\Delta})$. Hence we can define the desired mapping as the composition $\hat{\mathcal{S}}^{-1} \circ \bar{S}$. To check that this is an embedding it is enough to check that it preserves the layers (Lemma 11.4(vii)) and is isometric on the layers (Lemma 11.4(iii)).

To prove the last assertion fix $k$ and assume without loss of generality that $\sigma_{k}^{\min }=\hat{\sigma}_{k}$. Then $\left|\bar{v}_{k} \bar{w}_{k}\right| \geq\left|\widehat{v}_{k} \widehat{w}_{k}\right|-1 \geq 1$, as desired.

Now we prepare the statement of our final lemma. One can view it as a simple case of Theorem 10.1, case of $X$ being flat.

Let $\Delta$ be a characteristic disc for a thick interval $(i, j)$ for the directed geodesics $\left(\sigma_{k}\right),\left(\tau_{k}\right)$ between $\sigma, \tau$ and let $\gamma^{\prime}$ be its CAT(0) diagonal, cf Definition 9.10. Let $\left(\rho_{k}\right)_{k=i+1}^{j-1}$ be the simplices of the Euclidean diagonal in $\Delta$ (Definition 9.10). Fix $i \leq l<m \leq j$. If $i<l<m<j$ then let $\left(\alpha_{k}\right)_{k=l}^{m},\left(\beta_{k}\right)_{k=m}^{l}$ be directed geodesics in $\Delta$ from $\rho_{l}$ to $\rho_{m}$ and from $\rho_{m}$ to $\rho_{l}$ respectively. If $l=i$ then put $\rho_{i}=v_{i}$ in the definition of $\left(\alpha_{k}\right)_{k=l}^{m}$ and $\rho_{i}=w_{i}$ in the definition of $\left(\beta_{k}\right)_{k=m}^{l}$. If $m=j$ then put $\rho_{j}=w_{j}$ in the definition of $\left(\beta_{k}\right)_{k=m}^{l}$ and $\rho_{j}=v_{j}$ in the definition of $\left(\alpha_{k}\right)_{k=l}^{m}$. For all other purposes we will put $\rho_{i}=v_{i} w_{i}, \rho_{j}=v_{j} w_{j}$.

Let $\bigcup \widehat{\Delta}$ be the subcomplex of $\Delta$ which is the span of the union of $\operatorname{conv}\left\{\alpha_{k}, \beta_{k}\right\}$ over all $l \leq k \leq m$. Note that $\bigcup \widehat{\Delta}$ is a simplicial generalized characteristic disc. Denote the vertices of its boundary loop by $\left(\widehat{v}_{k}\right)$ and $\left(\widehat{w}_{k}\right)$. Denote by $\hat{\gamma}$ the $\operatorname{CAT}(0)$ geodesic joining in $\bigcup \widehat{\Delta}$ the barycenters of $\rho_{l}$ and $\rho_{m}$ (which lie in $\bigcup \widehat{\Delta}$ ).

Lemma 11.9 $\gamma^{\prime}$ restricted to $\left.\Delta\right|_{l} ^{m}$ and $\hat{\gamma}$ are $\frac{1}{2}$-close in $\left.\Delta\right|_{l} ^{m}$.

Proof We denote by $\bigcup \widehat{\Delta}_{0}$ the generalized characteristic disc obtained from $\bigcup \widehat{\Delta}$ by removing the following triangles: For any boundary vertex of defect 1 in the 
layers $\neq l, m$, say $\widehat{v}_{k}$, we cut off a triangle along the segment $\widehat{v}_{k-1} \widehat{v}_{k+1}$. For any boundary vertex of defect 2 (which is possible in the layers $l, m$ ), say $\hat{v}_{l}$, we cut off a triangle along the segment joining $\widehat{v}_{l+1}$ to the barycenter of $\widehat{v}_{l} \widehat{w}_{l}$.

We claim that $\bigcup \widehat{\Delta}_{0}$ is convex in $\Delta$ (treated as $\operatorname{CAT}(0)$ spaces). This means that at all vertices of $\partial \bigcup \widehat{\Delta}_{0}$ outside $\partial \Delta$, the interior angle of $\bigcup \widehat{\Delta}_{0}$ is at most $180^{\circ}$. We skip the proof, which is an easy consequence of Lemma 10.6.

Let $\hat{\gamma}_{0}$ be the CAT(0) geodesic in $\bigcup \widehat{\Delta}_{0}$ joining the barycenter $\hat{x}$ of $\rho_{l}$ with the barycenter $\hat{y}$ of $\rho_{m}$ (observe that $\hat{x}, \hat{y} \in \bigcup \widehat{\Delta}_{0}$ ). Since $\bigcup \widehat{\Delta}_{0} \subset \Delta$ is convex, $\widehat{\gamma}_{0}$ agrees with the $\operatorname{CAT}(0)$ geodesic in $\Delta$ joining $\hat{x}, \hat{y}$.

Now we apply Lemma 11.7 to $\left.\left.\Delta^{\prime}\right|_{l} ^{m} \subset \Delta\right|_{l} ^{m}$ (cf Definition 9.10 for the definition of $\Delta^{\prime}$ ), and geodesics $\hat{\gamma}_{0}$ in $\left.\Delta\right|_{l} ^{m}$ and $\gamma^{\prime}$ restricted to $\left.\Delta^{\prime}\right|_{l} ^{m}$. Observe that endpoints $\hat{x}, \hat{y}$ of $\hat{\gamma}_{0}$ are at distance at most $\frac{1}{2}$ from $\gamma^{\prime} \cap v_{l} w_{l}, \gamma^{\prime} \cap v_{m} w_{m}$ by the definition of $\rho_{l}, \rho_{m}$. Hence, by Lemma 11.7, we have that $\hat{\gamma}_{0}$ is $\frac{1}{2}$-close to $\gamma^{\prime}$ restricted to $\left.\Delta\right|_{l} ^{m}$.

Now observe that since $\bigcup \widehat{\Delta}_{0}$ is also convex in $\bigcup \widehat{\Delta}$, we have $\widehat{\gamma}_{0}=\widehat{\gamma}$ and we are done.

Finally, we can proceed with the following.

Proof of Theorem 10.1 First suppose that the layer $k$ for $\left(\sigma_{t}\right),\left(\tau_{t}\right)$ is thin. Then, by Proposition 10.2(i), $\widetilde{\sigma}_{k}$ contains or is contained in $\sigma_{k}$ and $\tilde{\tau}_{k}$ contains or is contained in $\tau_{k}$. Hence the thickness of the layer $k$ for $\left(\widetilde{\sigma}_{t}\right),\left(\tilde{\tau}_{t}\right)$ is at most 3 and thus $\widetilde{\sigma}_{k} \subset B_{1}\left(\tilde{\delta}_{k}\right)$ or $\tilde{\tau}_{k} \subset B_{1}\left(\tilde{\delta}_{k}\right)$, hence $\left|\tilde{\delta}_{k}, \delta_{k}\right| \leq 1$.

Now suppose that the layer $k$ for $\left(\sigma_{t}\right),\left(\tau_{t}\right)$ is thick and suppose it is contained in a thick interval $(i, j)$ with a characteristic disc $\Delta$. Put $\rho_{l}=v_{i} w_{i}$ if $l \leq i$ and $\rho_{m}=v_{j} w_{j}$ if $m \geq j$. We will use the notation introduced before Lemma 11.9. First suppose that the layer $k$ for $\left(\widetilde{\sigma}_{t}\right),\left(\tilde{\tau}_{t}\right)$ is thin. Then, by Proposition 10.2(ii), the maximal distance between vertices in $\mathcal{S}\left(\alpha_{k}\right)$ and $\mathcal{S}\left(\beta_{k}\right)$, hence (Lemma 9.8(iii)) in $\alpha_{k}$ and $\beta_{k}$ is at most 3. Since $\hat{\gamma} \cap v_{k} w_{k}$ lies in $\operatorname{conv}\left\{\alpha_{k}, \beta_{k}\right\}$, Lemma 11.9 implies that $\gamma^{\prime} \cap v_{k} w_{k}$ is at $\underset{\sim}{\text { distance at most }} \frac{1}{2}$ from $\operatorname{conv}\left\{\alpha_{k}, \beta_{k}\right\}$. Hence $\alpha_{k} \subset B_{1}\left(\rho_{k}\right)$ or $\beta_{k} \subset B_{1}\left(\rho_{k}\right)$. Thus $\tilde{\delta}_{k}, \delta_{k}$ are at distance at most 1 .

Now suppose that the layer $k$ for $\left(\widetilde{\sigma}_{t}\right),\left(\tilde{\tau}_{t}\right)$ is thick. Let $\widetilde{\Delta}$ be the characteristic disc for the thick interval $(\widetilde{i}, \widetilde{j})$ containing $k$ for $\left(\widetilde{\sigma}_{t}\right),\left(\tilde{\tau}_{t}\right)$. If the layer $k$ for $\left(\alpha_{t}\right),\left(\beta_{t}\right)$ (between $\rho_{l}, \rho_{m}$ in $\left.\Delta\right)$ is thin, then the thickness of the layer $k$ for $\left(\widetilde{\sigma}_{t}\right),\left(\tilde{\tau}_{t}\right)$ is at most 3, by Proposition 10.2(ii). Hence $\widetilde{\sigma}_{k} \subset B_{1}\left(\tilde{\delta}_{k}\right)$ or $\tilde{\tau}_{k} \subset B_{1}\left(\tilde{\delta}_{k}\right)$. By Lemma 11.9 we have $\left|\rho_{k}, \alpha_{k}\right| \leq 1$ and $\left|\rho_{k}, \beta_{k}\right| \leq 1$, hence altogether $\left|\tilde{\delta}_{k}, \delta_{k}\right| \leq 2$. 
So suppose that the layer $k$ for $\left(\alpha_{t}\right),\left(\beta_{t}\right)$ in $\Delta$ is thick, let $\hat{i}, \hat{j}$ be the thick interval for $\left(\alpha_{t}\right),\left(\beta_{t}\right)$ containing $k$ and let $\hat{\Delta}$ be the corresponding characteristic disc. Observe that $\widehat{\Delta}=\left.\bigcup \widehat{\Delta}\right|_{\hat{i}} ^{\hat{j}}$. Let $i_{\max }$ be the maximum of $\hat{i}, \widetilde{i}$ and $j_{\min }$ be the minimum of $\hat{j}, \tilde{j}$. Obviously $i_{\max }<k<j_{\min }$. Assume $i_{\max }+1<j_{\min }-1$, in the case of equality the argument is similar and we omit it.

By Proposition 10.2(ii) we can apply Lemma 11.8 to $\widetilde{\Delta}$ and $\widehat{\Delta}$ restricted to the interval $\left(i_{\max }+1, j_{\min }-1\right)$. Denote by $\bar{\Delta}$ the simplicial generalized characteristic disc for $\left(i_{\max }+1, j_{\min }-1\right)$ guaranteed by Lemma 11.8. Denote by $\bar{\Delta}^{\prime}$ the generalized characteristic disc obtained from $\bar{\Delta}$ by removing horizontal (the direction of $\bar{v}_{t} \bar{w}_{t}$ ) $\frac{1}{2}$-neighborhood of the boundary, which is allowed because $|\bar{v} \bar{w}| \geq 1$ by Lemma 11.8 . Let $\widetilde{\Delta}^{\prime}$ be the modified characteristic in $\widetilde{\Delta}$ and $\tilde{\gamma}^{\prime}$ the CAT(0) diagonal of $\widetilde{\Delta}$ (cf Definition 9.10). Define a generalized characteristic disc $\hat{\Delta}^{\prime} \subset \bigcup \widehat{\Delta}$ and a $\operatorname{CAT}(0)$ geodesic $\widehat{\gamma}^{\prime}$ in $\widehat{\Delta}^{\prime}$ as follows. For each $l \leq t \leq m$ denote by $\widehat{v}_{t}^{\prime}, \widehat{w}_{t}^{\prime}$ points on $\widehat{v}_{t} \widehat{w}_{t}$ at distance $\frac{1}{2}$ from $\widehat{v}_{t}, \widehat{w}_{t}$, respectively, if $\widehat{v}_{t} \neq \widehat{w}_{t}$. Otherwise, put $\widehat{v}_{t}^{\prime}=\widehat{v}_{t}, \widehat{w}_{t}^{\prime}=\widehat{w}_{t}$. Let $\widehat{\Delta}^{\prime}$ be the generalized characteristic disc enclosed by the loop $\widehat{v}_{l}^{\prime} \cdots \widehat{v}_{m}^{\prime} \widehat{w}_{m}^{\prime} \cdots \widehat{w}_{l}^{\prime} \widehat{v}_{l}^{\prime}$. Let $\widehat{\gamma}^{\prime}$ be the CAT(0) geodesic in $\widehat{\Delta}^{\prime}$ joining $\widehat{v}_{l}^{\prime}=\widehat{w}_{l}^{\prime}$ and $\widehat{v}_{m}^{\prime}=\widehat{w}_{m}^{\prime}$. By Lemma 11.8 (applied to restricted $\widetilde{\widetilde{\Delta}}$ and $\widehat{\Delta}$ ) we have inclusions of $\bar{\Delta}^{\prime}$ into $\left.\widehat{\Delta}^{\prime}\right|_{i_{\max }+1} ^{j_{\min }-1},\left.\widetilde{\Delta}^{\prime}\right|_{i_{\max }+1} ^{j_{\min }-1}$ with distances $\left|\bar{v}_{t}^{\prime} \widehat{v}_{t}^{\prime}\right|,\left|\bar{w}_{t}^{\prime} \widehat{w}_{t}^{\prime}\right|$ in $\widehat{\Delta}^{\prime}\left|\bar{v}_{t}^{\prime} \widetilde{v}_{t}^{\prime}\right|,\left|\bar{w}_{t}^{\prime} \widetilde{w}_{t}^{\prime}\right|$ in $\widetilde{\Delta}^{\prime}$ all at most 1 for $i_{\max }+1 \leq$ $t \leq j_{\min }-1$.

Now we will choose a special point $\bar{x} \in \bar{v}_{i_{\max }+1}^{\prime} \bar{w}_{i_{\max }+1}^{\prime}$. Without loss of generality assume $i_{\max }=\tilde{i}$, hence $\left|\tilde{v}_{i_{\max }+1} \tilde{w}_{i_{\max }+1}\right|=2$. Choose any $\bar{x}$ in $\bar{v}_{i_{\max }+1}^{\prime} \bar{w}_{i_{\max }+1}^{\prime}$ at distance $\leq 1$ from $\hat{\gamma}^{\prime}$, which is possible, since $\left|\bar{v}_{i_{\max }+1}^{\prime} \widehat{v}_{i_{\max }+1}^{\prime}\right| \leq 1$ and $\left|\bar{w}_{i_{\max }+1}^{\prime} \widehat{w}_{i_{\max }+1}^{\prime}\right| \leq 1$. Since $\mid \widetilde{v}_{i_{\max }+1}^{\prime} \widetilde{w}_{i_{\max }+1 \mid}^{\prime}=1, \bar{x}$ is also at distance at most 1 from $\widetilde{\gamma}^{\prime}$. Choose $\bar{y}$ in $\bar{v}_{j_{\min }-1}^{\prime} \bar{w}_{j_{\min }-1}^{\prime}$ in an analogous way.

By this construction the endpoints of $\tilde{\gamma}^{\prime}$ and $\hat{\gamma}^{\prime}$ restricted to $\left(i_{\max }+1, j_{\min }-1\right)$ are at distance at most 1 from $\bar{x}, \bar{y}$ in $\left.\widetilde{\Delta}^{\prime}\right|_{i_{\max }+1} ^{j_{\min }-1},\left.\widehat{\Delta}^{\prime}\right|_{i_{\max }+1} ^{j_{\min }-1}$, respectively. Thus, using twice Lemma 11.7, we get that $\tilde{\gamma}^{\prime}$ and $\widehat{\gamma}^{\prime}{ }_{\tilde{A}^{\max }}^{\text {restricted to }}\left(i_{\max }+1, j_{\min }-1\right)$ are $1-$ close to the CAT(0) geodesic $\bar{x} \bar{y}$ in $\bar{\Delta}^{\prime}$ (in $\left.\widetilde{\Delta}^{\prime}\right|_{i_{\max }+1} ^{j_{\min }-1},\left.\widehat{\Delta}^{\prime}\right|_{i_{\max }+1} ^{j_{\min }-1}$ respectively).

By Lemma 11.9, $\gamma^{\prime}$ and $\hat{\gamma}$ are $\frac{1}{2}$-close in $\left.\Delta\right|_{l} ^{m}$. By Lemma 11.7, $\hat{\gamma}^{\prime}$ and $\hat{\gamma}$ are $\frac{1}{2}$-close in $\widehat{\Delta} \mid \hat{\hat{j}}$. Putting those four estimates together we get that $\delta_{k}, \widetilde{\delta}_{k}$ are at distance at most 3 , as desired.

We end this section by indicating, how Theorem 10.1 can be promoted to Theorem B, with a reasonable constant $C$. The difference in statements comes from substituting $\delta_{l}, \delta_{m}$ with $x \in \delta_{l}, y \in \delta_{m}$ such that $|x y|=m-l$. As a first step, we check that Proposition 10.2 implies that the directed geodesics between $x$ and $y$ lie near the union of characteristic images of characteristic discs for $\left(\sigma_{k}\right),\left(\tau_{k}\right)$. This follows from the 
fact that directed geodesics in systolic complexes satisfy the so called fellow traveler property with a good constant; see Januszkiewicz-Świątkowski [19, Sections 11-12]. The second step is to reprove Lemma 11.8 allowing $\widehat{\sigma}_{k}$ and $\widetilde{\sigma}_{k}$ (and similarly $\hat{\tau}_{k}$ and $\tilde{\tau}_{k}$ ) to be farther apart, at distance bounded by the above fellow traveler constant. Then some minor changes in the proof of Theorem 10.1 yield Theorem B.

We will give a different complete proof of Theorem B (though with a worse constant) in the next section.

\section{Characteristic discs spanned on Euclidean geodesics}

In this section we prove the following crucial proposition, which, roughly speaking, says that in a characteristic disc spanned on a Euclidean geodesic and an arbitrary other geodesic, the boundary segment corresponding to the Euclidean geodesic is coarsely a CAT(0) geodesic. We introduce the following notation, which will be fixed for the whole section.

Let $\sigma, \tau$ be simplices in a systolic complex $X$ satisfying as before $\sigma \subset S_{n}(\tau), \tau \subset S_{n}(\sigma)$ and suppose that $\left(p_{k}\right)_{k=0}^{n},\left(r_{k}\right)_{k=0}^{n}$ are 1-skeleton geodesics with endpoints in $\sigma$ and $\tau$ such that $r_{k} \in \delta_{k}$, where $\left(\delta_{k}\right)_{k=0}^{n}$ is the Euclidean geodesic between $\sigma$ and $\tau$. Let $0 \leq i_{p r}<j_{p r} \leq n$ be a thick interval for $\left(p_{k}\right),\left(r_{k}\right)$ and let $\Delta_{p r}, \mathcal{S}_{p r}$ be the corresponding characteristic disc and mapping. Let $\gamma_{p r}$ be the CAT(0) geodesic in $\Delta_{p r}$ joining the barycenters of the unique edges in the layers $i_{p r}, j_{p r}$.

Proposition 12.1 $\gamma_{p r}$ is $97-$ close to the boundary path $\mathcal{S}_{p r}^{-1}\left(\left(r_{k}\right)\right)$.

This proposition has fundamental consequences. One of them is Theorem C, which says roughly this: in a "Euclidean geodesic triangle", the distance between the midpoints of two sides is, up to an additive constant, smaller than half of the length of the third side. We study this in the next section.

The second consequence of Proposition 12.1 is an alternative proof of the following.

Theorem 12.2 (Theorem B) Let $\sigma, \tau$ be simplices of a systolic complex $X$, such that for some natural $n$ we have $\sigma \subset S_{n}(\tau), \tau \subset S_{n}(\sigma)$. Let $\left(\delta_{k}\right)_{k=0}^{n}$ be the Euclidean geodesic between $\sigma$ and $\tau$. Take some $0 \leq l<m \leq n$ and let $\left(r_{k}\right)_{k=l}^{m}$ be $\underset{\tilde{\delta}_{l}}{1} 1-$ skeleton geodesic such that $r_{k} \in \delta_{k}$ for $l \leq k \leq m$. Consider the simplices $\tilde{\delta}_{l}=$ $r_{l}, \widetilde{\delta}_{l+1}, \ldots, \widetilde{\delta}_{m}=r_{m}$ of the Euclidean geodesic between vertices $r_{l}$ and $r_{m}$. Then for each $l \leq k \leq m$ we have $\left|\delta_{k}, \tilde{\delta}_{k}\right| \leq C$, where $C$ is a universal constant. 
Proof Extend $\left(r_{k}\right)_{k=l}^{m}$ to a 1-skeleton geodesic $\left(r_{k}\right)_{k=0}^{n}$ between $\sigma, \tau$ so that $r_{k} \in \delta_{k}$ (this is possible by Lemma 9.15(i)). Let $\left(\widetilde{r}_{k}\right)_{k=l}^{m}$ be any 1-skeleton geodesic between $r_{l}$ and $r_{m}$ such that $\widetilde{r}_{k} \in \tilde{\delta}_{k}$. Put additionally $\widetilde{r}_{k}=r_{k}$ for $0 \leq k<l$ and for $m<k \leq n$. Let $\Delta_{r} \tilde{r}$ be the characteristic disc for some thick interval for $\left(\widetilde{r}_{k}\right)_{k=0}^{n},\left(r_{k}\right)_{k=0}^{n}$ and let $\gamma_{r} \tilde{r}$ be the CAT(0) geodesic joining the barycenters of its outermost edges. Let $\mathcal{S}_{r} \tilde{r}$ be the corresponding characteristic mapping.

Notice that $\Delta_{r} \tilde{r}$ is also a characteristic disc for $\left(r_{k}\right)_{k=l}^{m},\left(\widetilde{r}_{k}\right)_{k=l}^{m}$ between $r_{l}$ and $r_{m}$. Applying twice Proposition 12.1 we obtain that $\gamma_{r} \tilde{r}$ is 97-close to both $\mathcal{S}_{r \tilde{r}}^{-1}\left(\left(r_{k}\right)\right)$ and $\mathcal{S}_{r \tilde{r}}^{-1}\left(\left(\widetilde{r}_{k}\right)\right)$. This proves that for all $l \leq k \leq m$ we have $\left|r_{k} \widetilde{r}_{k}\right| \leq 194$, hence $\left|\delta_{k}, \tilde{\delta_{k}}\right| \leq 194$. Thus any $C \geq 194$ satisfies the assertion of the theorem.

The proof of Proposition 12.1 is rather technical. This is the reason we decided to present the straightforward proof of Theorem 10.1 (the weak version of Theorem B) via Proposition 10.2. Before we get into technical details of the proof, split into various lemmas, we present an outline, which hopefully helps to keep track of the main ideas.

Outline of the proof of Proposition 12.1 We are dealing with configurations of four geodesics between $\sigma$ and $\tau$ : the directed geodesics, denoted by $\left(\sigma_{k}\right)_{k=0}^{n},\left(\tau_{k}\right)_{k=0}^{n}$ as in the previous sections, $\left(r_{k}\right)_{k=0}^{n}$, which goes along the Euclidean geodesic $\delta_{k}$, and the fourth arbitrary 1-skeleton geodesic $\left(p_{k}\right)_{k=0}^{n}$. For the layer $k$ thick (for $\left(\sigma_{k}\right),\left(\tau_{k}\right)$ ) we have that $\delta_{k}=\mathcal{S}\left(\rho_{k}\right)$, where $\rho_{k}$ is the simplex of the Euclidean diagonal in appropriate characteristic disc $\Delta$ for $\left(\sigma_{k}\right),\left(\tau_{k}\right)$. Hence we need to find out, what is the possible position of $\left(p_{k}\right)$ with respect to $\mathcal{S}(\Delta)$. It turns out that in each layer there are 1-skeleton geodesics between simplices $\sigma_{k}, \tau_{k}$ and $p_{k}$, which form a very thin triangle (Lemma 12.3). The intersection with $\mathcal{S}(\Delta)$ of the center simplex of this triangle will be later denoted by $\bar{\chi}_{k}$.

In Lemma 12.4 we study, how do $\bar{\chi}_{k}$ vary with $k$. Assume for simplicity that $p_{k}$ stay away from $\mathcal{S}(\Delta)$. Then it turns out that first (ie for small $k$ ) $\bar{\chi}_{k}$ follow $\mathcal{S}\left(w_{k}\right)$, next the barycenters of $\bar{\chi}_{k}$ lie in the characteristic image of a vertical line in $\Delta$ and last $\bar{\chi}_{k}$ follow $\mathcal{S}\left(v_{k}\right)$. The CAT( 0$)$ diagonal $\gamma^{\prime}$ of $\Delta$ crosses this line at most once. Thus we can divide each "thick" interval (an interval with all layers thick, in opposition to the thick interval with thin endpoint layers) for $\left(\sigma_{k}\right),\left(\tau_{k}\right)$ into three subintervals: the "initial" one, for which $\chi_{k}=\mathcal{S}^{-1}\left(\bar{\chi}_{k}\right)$ is far to the right from $\rho_{k}$ or near $w_{k} \in \partial \Delta$, the "middle" one, for which $\chi_{k}$ is near $\rho_{k}$, and the "final" one, for which $\chi_{k}$ is far to the left from $\rho_{k}$ or near $v_{k} \in \partial \Delta$; see Lemma 12.8. Moreover, in the "initial" (resp. "final") interval we can distinguish a "preinitial" (resp. "postfinal") interval in which $\gamma^{\prime}$ stays away from $w_{k}^{\prime} \in \partial \Delta^{\prime}$ (resp. $v_{k}^{\prime} \in \partial \Delta^{\prime}$ ), where $\Delta^{\prime}$ is the modified characteristic disc. This distinction is done in the main body of the proof of Proposition 12.1. The 
vertices $\mathcal{S}_{p r}^{-1}\left(r_{k}\right)$ in $\Delta_{p r}$, for $k$ in one of these intervals, are positioned as follows. The vertices of the "middle" interval together with the vertices of the other ones outside the "preinitial" and "postfinal" intervals form a coarse vertical line (this is a consequence of Lemma 12.9), while the vertices of the "preinitial" and "postfinal" intervals form also coarse CAT(0) geodesics, fortunately forming with the coarse vertical line angles $\geq 180^{\circ}$ at the endpoints. This proves Proposition 12.1 in the simple case of a single "thick" interval for $\left(\sigma_{k}\right),\left(\tau_{k}\right)$.

In the complex case, the question is, how may the various "thick" intervals and thin layers for $\left(\sigma_{k}\right),\left(\tau_{k}\right)$ alternate. We define roughly the following notions. A "thin" interval is an interval of not very thick layers. A "proper thin" interval is a "thin" interval with thin layers at the beginning and at the end. A "very thick" interval is an interval containing a layer that is very thick. In Lemma 12.11 we prove that the vertices $\mathcal{S}_{p r}^{-1}\left(r_{k}\right)$, for $k$ in a "thin" interval, form a coarse vertical line. In Corollary 12.10 we prove that if at the beginning of a thin layer there is an adjoined "thick" interval, then this "thick" interval has the "final" subinterval constructed above "thin". Similarly, if at the end of a thin layer there is an adjoined "thick" interval, then this thick interval has the "initial" subinterval "thin". The last piece of the puzzle is an assertion in Lemma 12.8, that for a "very thick" interval, either its "initial" or "final" subinterval is non-"thin".

The way to put these pieces together is the following. We take a maximal "proper thin" interval. The "very thick" interval adjoined at the beginning of this "proper thin" interval must have either the "initial" or the "final" subinterval non-"thin" (Lemma 12.8), but the possibility of the "final" subinterval non-"thin" is excluded (Corollary 12.10). Thus its "initial" subinterval is non-"thin" and this excludes the possibility that some thin layer (hence any layer) is adjoined at the beginning of this "very thick" interval (Corollary 12.10). We can apply analogous considerations to the "very thick" interval adjoined at the end of the "proper thin" interval. Altogether, we have the following configuration: the "proper thin" interval, with a "very thick" interval with "thin" "final" subinterval adjoined at the beginning, and with a "very thick" interval with "thin" "initial" subinterval adjoined at the end. Moreover, in the first of the "very thick" intervals we distinguish the "preinitial" interval and in the second one we distinguish the "postfinal" interval. The vertices $\mathcal{S}_{p r}^{-1}\left(r_{k}\right)$, for $k$ outside the "preinitial" and "postfinal" intervals, form a coarse vertical line (Lemma 12.9 and Lemma 12.11), and the ones for $k$ in the "preinitial" and "postfinal" intervals form also coarse CAT(0) geodesics forming with the coarse vertical line angles $\geq 180^{\circ}$ at the endpoints. This ends the outline of the proof of Proposition 12.1.

The following lemma treats configurations of three vertices in a layer. Denote the layers between $\sigma, \tau$ by $L_{k}$. 
Lemma 12.3 Suppose $p, s, t$ are three vertices in $L_{k}$. Then either there exists a vertex such that there are 1-skeleton geodesics $p s, p t$, st passing through this vertex or there exists a triangle (ie a 2-simplex) such that there are 1-skeleton geodesics $p s, p t, s t$ passing through the edges of this triangle.

Proof Let $p^{\prime}$ be a vertex farthest from $p$ lying both on some 1-skeleton geodesic $p s$ and some 1-skeleton geodesic $p t$. Let $s^{\prime}$ be a vertex farthest from $s$ lying both on some 1-skeleton geodesic $s p^{\prime}$ and some 1-skeleton geodesic $s t$. Finally let $t^{\prime}$ be a vertex farthest from $t$ lying both on some 1-skeleton geodesic $t p^{\prime}$ and some $1-$ skeleton geodesic $t s^{\prime}$. If two of the vertices $p^{\prime}, s^{\prime}, t^{\prime}$ coincide, then all three coincide and the lemma follows immediately. Suppose now that those three vertices are distinct.

From the choice of $p^{\prime}, s^{\prime}, t^{\prime}$ it follows that any loop $\Gamma$ obtained by concatenating some $1-$ skeleton geodesics $p^{\prime} s^{\prime}, s^{\prime} t^{\prime}, t^{\prime} p^{\prime}$ is embedded in $L_{k}$. Since $L_{k}$ is convex (Remark 8.2), it is contractible (see remarks after Definition 2.4), hence $\Gamma$ is contractible in $L_{k}$ (we could also invoke Lemma 8.4). Consider a surface $T: D \rightarrow L_{k}$ of minimal area spanned on such a geodesic triangle $\Gamma$ (we allow the geodesics to vary). By minimality of area the defects at interior vertices of $D$ and at interior vertices of the boundary geodesics are nonpositive. Since by Gauss-Bonnet Lemma 7.2 the total sum of defects equals 6 , we get that all mentioned vertices have defects 0 and the vertices of the geodesic triangle $D$ have defect 2 . Hence $D$ is a subcomplex of $\mathbb{E}_{\Delta}^{2}$ which is a Euclidean equilateral triangle. Denote the length of the side of this triangle by $d>0$. If $d \geq 2$ then let $u$ be the vertex in $D$ such that $T(u)=p^{\prime}$, let $u_{1}, u_{2}$ be its neighbors in $D$, let $u_{3}$ be the common neighbor of $u_{1}, u_{2}$ in $D$ different from $u$ and let $u_{4}$ be the neighbor of $u_{1}$ different from previously mentioned vertices. By Corollary 8.6 applied to the trapezoid $T(u) T\left(u_{1}\right) T\left(u_{2}\right) T\left(u_{3}\right) T\left(u_{4}\right)$ either we have an edge $T(u) T\left(u_{3}\right)$ or $T\left(u_{2}\right) T\left(u_{4}\right)$. In the first case the vertex $T\left(u_{3}\right)$ turns out to lie on some 1 -skeleton geodesics $s p, t p$ contradicting the choice of $p^{\prime}$. In the second case the vertex $T\left(u_{2}\right)$ turns out to lie some 1 -skeleton geodesics $s p, t p$, also giving a contradiction. Hence $d=1$ and the lemma follows.

In the next lemma we analyze the possible position of $\left(p_{k}\right)$ with respect to the partial characteristic image $\mathcal{S}(\Delta)$ of a partial characteristic disc $\Delta$ for $(i, j)$ for $\left(\sigma_{k}\right),\left(\tau_{k}\right)$. This means that we assume that the layers $i \leq k \leq j$ are thick, cf Definition 11.3. In the language of the outline of the proof of Proposition 12.1 this is the "thick" interval. The boundary vertices of $\Delta$ are, as always, denoted by $\left(v_{k}\right),\left(w_{k}\right)$.

For each $i \leq k \leq j$ let $s_{k} \in \sigma_{k}, t_{k} \in \tau_{k}$ be chosen as in the previous sections to maximize the distance $\left|s_{k} t_{k}\right|$. Moreover, among those, choose $s_{k}, t_{k}$ to maximize the distances $\left|p_{k} s_{k}\right|,\left|p_{k} t_{k}\right|$ (it is possible to do this independently by Lemma 9.5). For each $k$ 
perform in $L_{k}$ the construction of $s_{k}^{\prime}, t_{k}^{\prime}, p_{k}^{\prime}$ as in the proof of Lemma 12.3 and denote $\bar{\chi}_{k}=s_{k}^{\prime} t_{k}^{\prime}$, which is an edge or a vertex in some 1 -skeleton geodesic $s_{k} t_{k}$. Denote $\chi_{k}=\mathcal{S}^{-1}\left(\bar{\chi}_{k}\right)$. Observe that $\chi_{k}$ does not depend on the choice of $s_{k}, t_{k}, s_{k}^{\prime}, t_{k}^{\prime}, p_{k}^{\prime}$, since it is determined by the distances $\left|s_{k} t_{k}\right|,\left|s_{k} p_{k}\right|,\left|t_{k} p_{k}\right|$. Lemmas 12.4-12.8 are devoted to studying the position of $\chi_{k}$ with respect to $\rho_{k}$ (the simplices of the Euclidean diagonal).

The paths $\left(v_{k}\right),\left(w_{k}\right)$ are the boundary components of $\Delta$.

Finally, note that in the lemma below we actually do not have to assume that $\left(\sigma_{k}\right),\left(\tau_{k}\right)$ are directed geodesics.

Lemma 12.4 In the above setting, assume that for all $i \leq k \leq j$ we have $p_{k} \neq p_{k}^{\prime}$ (this does not depend on the choice of $p_{k}^{\prime}$ ). Then for $i \leq k<j$,

(i) if $\chi_{k}, \chi_{k+1}$ are both edges, then they both intersect the same boundary component,

(ii) if one of $\chi_{k}, \chi_{k+1}$, say $\chi_{k}$, is an edge, and the second is a vertex, then either $\chi_{k}, \chi_{k+1}$ span a simplex, or they intersect the same boundary component,

(iii) if $\chi_{k}, \chi_{k+1}$ are both vertices, then they both lie on the same boundary component.

If we remove the assumption that $p_{k} \neq p_{k}^{\prime}$, then in case (i) we only have that $\chi_{k} \subset$ $S_{1}\left(\chi_{k+1}\right)$ and $\chi_{k+1} \subset S_{1}\left(\chi_{k}\right)$, case (ii) remains unchanged, and in case (iii) we only have that $\chi_{k}, \chi_{k+1}$ span an edge.

Proof We first prove the last assertion. We need to prove (up to interchanging $k$ with $k+1$ ) that for a vertex $u_{0} \in \chi_{k}$ either there exists a neighbor of $u_{0}$ in $\chi_{k+1}$, or $\chi_{k}, \chi_{k+1}$ intersect the same boundary component. Suppose the first part of this alternative does not hold. Then, up to interchanging $v_{k}$ with $w_{k}$, we have the following configuration (which it will take some time to describe, since we need to name all the vertices that come into play):

We have $u_{0} \neq w_{k}$, and we denote by $u_{1}$ the vertex following $u_{0}$ on 1 -skeleton geodesic in $\Delta$ from $u_{0}$ to $w_{k}$, and by $u_{2}$ the vertex following $u_{1}$ if $u_{1} \neq w_{k}$. In the layer $k+1$ we denote by $z_{1} \neq w_{k+1}$ the vertex in the residue of $u_{0} u_{1}$ and by $z_{2}$ the vertex following $z_{1}$ on 1 -skeleton geodesic $z_{1} w_{k+1}$. The configuration is the following: $\chi_{k+1}$ lies on the 1 -skeleton geodesic $z_{2} w_{k+1}$.

Fix some 1-skeleton geodesics $s_{l} \cdots s_{l}^{\prime}, t_{l} \cdots t_{l}^{\prime}, p_{l} \cdots p_{l}^{\prime}$ for $l=k, k+1$. Consider a partial characteristic surface $S: \Delta \rightarrow X$ such that for $l=k, k+1$ we have that $S\left(v_{l} w_{l}\right)$ 
(where $v_{l} w_{l}$ is the 1 -skeleton geodesic in $\Delta$ ) contains $s_{l} \cdots s_{l}^{\prime}$ and $t_{l}^{\prime} \cdots t_{l}$ (this is possible by Proposition 7.6). Then $S\left(z_{2}\right) \in s_{k+1} \cdots s_{k+1}^{\prime} \subset s_{k+1} \cdots s_{k+1}^{\prime} p_{k+1}^{\prime} \cdots p_{k+1}$ (where possibly $s_{k+1}^{\prime}=p_{k+1}^{\prime}$ ). By Proposition 7.6 applied to the partial characteristic surface for $p_{k}, p_{k+1}, s_{k}, s_{k+1}$ containing $s_{k} \cdots s_{k}^{\prime}$, there is a neighbor of $S\left(z_{2}\right)$ on $s_{k} \cdots s_{k}^{\prime} p_{k}^{\prime} \cdots p_{k}$ (where possibly $s_{k}^{\prime}=p_{k}^{\prime}$ ). Denote this neighbor by $\bar{x}$. Since $S\left(u_{0}\right) \in \bar{\chi}_{k}$, we have that $\bar{x} \neq S\left(u_{1}\right), \bar{x} \neq S\left(u_{2}\right)$. Moreover, since the vertices in the 1 -skeleton geodesic $v_{k} u_{0}$ are not neighbors of $z_{2}$, we have by Lemma 11.4(iii) that $\bar{x} \notin s_{k} \cdots s_{k}^{\prime}$. So $\bar{x} \in p_{k}^{\prime} \cdots p_{k}$. But by Lemma 2.8 the vertices $\bar{x}, S\left(u_{1}\right)$, together with $S\left(u_{2}\right)$, if defined, span a simplex. On the other hand, $S\left(u_{1}\right)$, and $S\left(u_{2}\right)$ if defined, lie on the 1 -skeleton geodesic $p_{k} \cdots p_{k}^{\prime} t_{k}^{\prime} \cdots t_{k}$ passing through $\bar{x}$. Since $\bar{x}, S\left(u_{1}\right)$, and $S\left(u_{2}\right)$, if defined, are different vertices, this is only possible if $\bar{x}=p_{k}^{\prime}, S\left(u_{0}\right)=s_{k}^{\prime}, S\left(u_{1}\right)=t_{k}^{\prime}$ and $u_{1}=w_{k}$, ie $u_{2}$ is not defined. Then $\chi_{k}$ is an edge, $\chi_{k+1}$ is a vertex, and they intersect the same boundary component, which is the second possibility of the alternative. Thus we have proved the last assertion of the lemma. In particular, we have proved assertion (ii).

Now we will be proving assertions (i) and (iii) and we may already assume that $p_{k} \neq p_{k}^{\prime}$ for $i \leq k \leq j$.

First we prove (i), by contradiction. Suppose that $\chi_{k}, \chi_{k+1}$ are both edges, and without loss of generality suppose that $\chi_{k}$ does not intersect the boundary. This implies that $s_{k}^{\prime} \neq s_{k}, t_{k}^{\prime} \neq t_{k}$. Let $\bar{z}$ be a vertex in the projection (cf Definition 2.9) of the triangle $s_{k}^{\prime} t_{k}^{\prime} p_{k}^{\prime}$ onto the layer $L_{k+1}$. By Lemma 10.5 applied thrice we get that $\bar{z}$ lies on 1skeleton geodesics between all pairs of vertices from $\left\{s_{k+1}, t_{k+1}, p_{k+1}\right\}$, thus $\bar{\chi}_{k+1}$ is a vertex. Contradiction.

Now we prove (iii), by contradiction. Suppose that $\chi_{k}, \chi_{k+1}$ are both vertices and one of them is nonboundary. Then in the layers $k, k+1$ of $\Delta$ there are vertices, which are common neighbors of $\chi_{k}, \chi_{k+1}$, denote them by $u$ (in the layer $k$ ) and by $z$ (in the layer $k+1$ ). Moreover, either $\chi_{k} \neq v_{k}$ and $\chi_{k+1} \neq v_{k+1}$, or $\chi_{k} \neq$ $w_{k}$ and $\chi_{k+1} \neq w_{k+1}$. Assume without loss of generality that the latter holds. Consider the partial characteristic disc $\Delta_{p t}$ for $p_{k}, p_{k+1}, t_{k}, t_{k+1}$ (we are allowed to do this, since $\left|p_{k} t_{k}\right|=\left|p_{k} \bar{\chi}_{k}\right|+\left|\bar{\chi}_{k} t_{k}\right| \geq 2$ and similarly $\left|p_{k+1} t_{k+1}\right| \geq 2$ ) and the corresponding partial characteristic mapping $\mathcal{S}_{p t}$. Let $x$ be the common neighbor of $\mathcal{S}_{p t}^{-1}\left(\bar{\chi}_{k}\right), \mathcal{S}_{p t}^{-1}\left(\bar{\chi}_{k+1}\right)$ in $\Delta_{p t}$ lying on $\mathcal{S}_{p t}^{-1}\left(p_{k} \bar{\chi}_{k}\right)$ or $\mathcal{S}_{p t}^{-1}\left(p_{k+1} \bar{\chi}_{k+1}\right)$. Assume, without loss of generality, that $\mathcal{S}_{p t}(x) \subset L_{k}$. Since vertices in $\mathcal{S}_{p t}(x), \mathcal{S}(u) \subset L_{k}$ are neighbors of $\bar{\chi}_{k+1} \in L_{k+1}$, we have by Lemma 2.8 that $\mathcal{S}_{p t}(x)$ and $\mathcal{S}(u)$ span a simplex. On the other hand, $\bar{\chi}_{k}$ lies by definition on some $1-$ skeleton geodesic $p_{k} s_{k}$. By Proposition 7.6, its segments $p_{k} \bar{\chi}_{k}$ and $\bar{\chi}_{k} s_{k}$ intersect $\mathcal{S}_{p t}(x)$ and $\mathcal{S}(u)$, respectively (outside $\bar{\chi}_{k}$ ). Hence $\bar{\chi}_{k}$ separates vertices from $\mathcal{S}_{p t}(x)$ and $\mathcal{S}(u)$ on a 
1-skeleton geodesic $p_{k} s_{k}$. Contradiction. Thus we have proved assertion (iii) and hence the whole lemma.

Let us introduce the following language.

Definition 12.5 We will refer to the horizontal coordinates of points in various characteristic discs. Namely, we view a characteristic disc as a CAT(0) subspace of $\mathbb{E}^{2}$. There we consider cartesian coordinates such that the layers are contained in horizontal lines. We also specify that the horizontal coordinate increases (from the left to the right) in the direction from $v_{k}$ to $w_{k}$. We denote the horizontal coordinate of a point $z$ by $z^{x}$. If $\lambda$ is a vertical line in $\Delta$, then its horizontal coordinate is denoted by $\lambda^{x}$.

We will need the following technical lemma, which helps to compare the horizontal coordinates of the preimages of vertices of $X$ in various characteristic discs.

Lemma 12.6 Suppose that $\Delta^{1}, \Delta^{2}$ are partial characteristic discs (and $\mathcal{S}^{1}, \mathcal{S}^{2}$ resp. characteristic mappings) for the interval $(i, j)$ for some sequences of simplices $\left(\sigma_{k}^{1}\right)$, $\left(\tau_{k}^{1}\right),\left(\sigma_{k}^{2}\right),\left(\tau_{k}^{2}\right)$ in the layers $L_{k}$ between $\sigma, \tau$. Suppose $\left(p_{k}\right)_{k=i}^{j},\left(\tilde{p}_{k}\right)_{k=i}^{j}$ are $1-$ skeleton geodesics such that for $i \leq k \leq j$ we have that $p_{k}, \tilde{p}_{k} \in L_{k}$ and, for $l=1,2$, we have $p_{k}, \tilde{p}_{k} \in \mathcal{S}^{l}\left(\Delta^{l}\right)$ but $\left(\mathcal{S}^{l}\right)^{-1}\left(p_{k}\right) \neq\left(\mathcal{S}^{l}\right)^{-1}\left(\tilde{p}_{k}\right)$. Then, if we vary $i \leq k \leq j$, the differences within $\left(\left(\mathcal{S}^{1}\right)^{-1}\left(p_{k}\right)\right)^{x}$ and within $\left(\left(\mathcal{S}^{2}\right)^{-1}\left(p_{k}\right)\right)^{x}$ agree.

Proof Apply Lemma 11.4(iii).

The following notions will help us formulate neatly the upcoming lemma.

Definition 12.7 Let $\Delta$ be a simplicial generalized characteristic disc for $(i, j)$ such that $\left|v_{k} w_{k}\right| \geq 2$ for $i \leq k \leq j$. Let $\chi, \rho$ be some simplices in the layer $k$ of $\Delta$, and $c \in \mathbb{Z}_{+}$. We say that $\chi$ is

- $\partial$-left if either $v_{k} \in \chi$ or $\chi$ is a neighbor vertex of $v_{k}$, which has defect 1 in case $k \neq i, j$ or defect 2 in case $k=i$ or $k=j$,

- $\partial$-right if either $w_{k} \in \chi$ or $\chi$ is a neighbor vertex of $w_{k}$, which has defect 1 in case $k \neq i, j$ or defect 2 in case $k=i$ or $k=j$,

- $c$-left from $\rho$ if $|\chi, \rho| \geq c$ and $\chi$ lies on $v_{k} \rho$,

- $c$-right from $\rho$ if $|\chi, \rho| \geq c$ and $\chi$ lies on $\rho w_{k}$.

In all that follows, $c$ is a positive integer. When all the pieces of the proof of Proposition 12.1 are put together, we assign $c=5$. But before this happens, we use the variable $c$, in order to help keeping track of the role of the constant in the various lemmas. 
Lemma 12.8 Assume that for some $i<j$ and each $i \leq k \leq j$ the layer $k$ is thick for $\left(\sigma_{k}\right),\left(\tau_{k}\right)$, and $\left|p_{k}, \delta_{k}\right| \geq c+4$. Then there exist $i \leq l \leq m \leq j$ such that

(i) for $i \leq k<l$ we have that $\chi_{k}$ is $\partial$-right or $c$-right from $\rho_{k}$,

(ii) among $l \leq k \leq m$ the differences within $\left(\mathcal{S}_{p r}^{-1}\left(r_{k}\right)\right)^{x}$ are $\leq c+1$,

(iii) for $m<k \leq j$ we have that $\chi_{k}$ is $\partial$-left or $c$-left from $\rho_{k}$.

Moreover, if the maximal thickness of the layers (for $\left(\sigma_{k}\right),\left(\tau_{k}\right)$ ) from $i$ to $j$ is at least $2 c+4$ and the layers $i-1, j+1$ are thin, then there are $l, m$ as above such that either $m<j$ and $v_{j+1}^{x}-v_{m+1}^{x} \geq c$ (in the characteristic disc for the thick interval $(i-1, j+1))$ or $l>i$ and $w_{l-1}^{x}-w_{i-1}^{x} \geq c$.

The ranges for $k$ in (i), (ii) and (iii), define the "initial" subinterval, the "middle" subinterval and the "final" subinterval of a "thick" interval discussed in the outline of the proof of Proposition 12.1. The last assertion, in the language of the outline, states that a "very thick" interval has either its "initial" or "final" subinterval non-"thin".

Proof First we give the proof of (i)-(iii) under an additional assumption that for all $i \leq k \leq j$ we have $p_{k} \neq p_{k}^{\prime}$ (recall that this does not depend on the choice of $p_{k}^{\prime}$ ). The outline of the proof with this assumption was already given at the beginning of the section.

To start, observe that from Lemma 12.4 and Lemma 9.16(i)-(ii) we get immediately the following.

Corollary There exist $i \leq l^{\prime} \leq m^{\prime} \leq j$ such that

(1) for $i \leq k<l^{\prime}$ the simplex $\chi_{k}$ is $\partial$-right,

(2) for $l^{\prime} \leq k \leq m^{\prime}$ the simplices $\chi_{k}$ are alternatingly edges and vertices and their barycenters lie on a straight vertical line $\lambda$ in $\Delta$; moreover for $l^{\prime}<k<m^{\prime}$ the simplices $\chi_{k}$ do not meet $v_{k}, w_{k}$,

(3) for $m^{\prime}<k \leq j$ the simplex $\chi_{k}$ is $\partial-$ left.

Recall that the restriction to $\Delta$ (the partial characteristic disc for $(i, j)$ for $\left(\sigma_{k}\right),\left(\tau_{k}\right)$ ) of the CAT(0) diagonal $\gamma^{\prime}$ (cf Definition 9.10) in the characteristic disc (Definition 9.4) containing $\Delta$ crosses transversally each vertical line in $\Delta$, by Lemma 9.17 (since $(j+1)-(i-1)>2)$. Let $l^{\prime} \leq l \leq m^{\prime}$ be maximal satisfying $\left(\gamma^{\prime} \cap v_{k} w_{k}\right)^{x} \leq \lambda^{x}-c-\frac{1}{2}$ for $l^{\prime} \leq k<l$. Similarly, let $l^{\prime} \leq m \leq m^{\prime}$ be minimal satisfying $\left(\gamma^{\prime} \cap v_{k} w_{k}\right)^{x} \geq \lambda^{x}+c+\frac{1}{2}$ for $m<k \leq m^{\prime}$. 
We prove that assertion (i) is satisfied with $l$ as above. First consider $i \leq k<l^{\prime}$. Then assertion (i) follows from assertion (1) of the corollary. Now suppose that $l^{\prime} \leq k<l$. Then, by the definitions of $l$ and $\rho_{k}$, if $\rho_{k}$ is a vertex, then $\rho_{k}^{x} \leq \lambda^{x}-c-\frac{1}{2}$, and if $\rho_{k}$ is an edge then the horizontal coordinates of its vertices are at most $\lambda^{x}-c$. Moreover, in case the latter inequality is an equality, we have that $\chi_{k}$ is a vertex. In all cases $\chi_{k}$ lies to the right of $\rho_{k}$ and the distance between them is at least $c$, as desired. Analogously, assertion (iii) holds with $m$ as above.

Now we prove assertion (ii). Consider $l \leq k \leq m$. If $l=m=l^{\prime}$ or $l=m=m^{\prime}$, then (ii) follows immediately. Otherwise, by the definition of $m, l$ we have $\left(\gamma^{\prime} \cap v_{l} w_{l}\right)^{x}>$ $\lambda^{x}-c-\frac{1}{2}$ and $\left(\gamma^{\prime} \cap v_{m} w_{m}\right)^{x}<\lambda^{x}+c+\frac{1}{2}$, hence $\lambda^{x}-c-\frac{1}{2}<\left(\gamma^{\prime} \cap v_{k} w_{k}\right)^{x}<\lambda^{x}+c+\frac{1}{2}$. By the definition of $\rho_{k}$, via similar considerations as in the previous paragraph, we have that $\operatorname{diam}\left(\rho_{k} \cup \chi_{k}\right) \leq c+1$ and $\left|\rho_{k}, \chi_{k}\right| \leq c$. By the former inequality we have that $p_{k}^{\prime}$ are at distance at most $c+1$ from $r_{k}$. (Record the latter one, ie $\left|\rho_{k}, \chi_{k}\right| \leq c$, which we will need later in the proof.)

We would like to compute the differences within $\left(\mathcal{S}_{p r}^{-1}\left(p_{k}^{\prime}\right)\right)^{x}$, when we vary $l \leq k \leq m$. These differences are equal to the differences within $\left(\mathcal{S}_{p s}^{-1}\left(p_{k}^{\prime}\right)\right)^{x}$ in $\Delta_{p s}$, where $\mathcal{S}_{p s}$ (resp. $\Delta_{p s}$ ) is the partial characteristic mapping (resp. partial characteristic disc) for $\left(p_{k}\right)_{k=l}^{m},\left(s_{k}\right)_{k=l}^{m}$. To see this, it is enough to apply Lemma 12.6 with $\left(p_{k}^{\prime}\right),\left(p_{k}\right)$ in place of $\left(p_{k}\right),\left(\tilde{p}_{k}\right)$, where we use our additional assumption $p_{k} \neq p_{k}^{\prime}$.

We claim that $\left(\mathcal{S}_{p s}^{-1}\left(p_{k}^{\prime}\right)\right)^{x}$ vary at most by $\frac{1}{2}$ for $l \leq k \leq m$. Indeed, by our additional assumption and assertion (2) of the corollary we have, for $l<k<m$, that $p_{k} \neq$ $p_{k}^{\prime}, s_{k} \neq s_{k}^{\prime}, t_{k} \neq t_{k}^{\prime}$. Thus we can apply Lemma 12.4 with $\left(s_{k}\right),\left(p_{k}\right),\left(t_{k}\right)$ in place of $\left(\sigma_{k}\right),\left(\tau_{k}\right),\left(p_{k}\right)$ to obtain, for $l \leq k \leq m$, that the barycenters of $\mathcal{S}_{p s}^{-1}\left(p_{k}^{\prime} s_{k}^{\prime}\right)$ lie on a common vertical line in $\Delta_{p s}$. This justifies the claim.

Thus $\left(\mathcal{S}_{p r}^{-1}\left(p_{k}^{\prime}\right)\right)^{x}$ vary at most by $\frac{1}{2}$, for $l \leq k \leq m$. Let $\mu$ be the greater among (at most two) values attained by $\left(\mathcal{S}_{p r}^{-1}\left(p_{k}^{\prime}\right)\right)^{x}$. By the previous estimates we have that $\left(\mathcal{S}_{p r}^{-1}\left(r_{k}\right)\right)^{x} \leq \mu+c+1$. On the other hand, we have $\mu \leq\left(\mathcal{S}_{p r}^{-1}\left(r_{k}\right)\right)^{x}$. Hence we obtain that the differences within $\left(\mathcal{S}_{p r}^{-1}\left(r_{k}\right)\right)^{x}$ are $\leq c+1$, as desired.

Now we must remove the additional assumption that for all $i \leq k \leq j$ we have $p_{k} \neq p_{k}^{\prime}$. We have now only the last assertion of Lemma 12.4 at our disposal.

Let $i \leq i_{1} \leq j_{1}<i_{2} \leq j_{2}<\cdots<i_{q} \leq j_{q} \leq j$, where $j_{h}<i_{h+1}-1$ for $1 \leq h<q$, be such that exactly for $i_{h} \leq k \leq j_{h}$ our additional assumption is satisfied. For all other $i \leq k \leq j$, in particular, for $k=i_{h}-1, j_{h}+1$ (where $1 \leq h \leq q$ ), except possibly for $i_{1}-1$ if it equals $i-1$, and $j_{q}+1$ if it equals $j+1$, we have $\left|\chi_{k}, \rho_{k}\right| \geq\left|p_{k}, \delta_{k}\right|-1 \geq c+3$. Thus for $k=i_{h}, j_{h}$, except possibly for $i_{1}$ if it equals $i$ and for $j_{q}$ if it equals $j$, we have, by Lemma 9.11 and by the last assertion of Lemma 12.4, that $\left|\chi_{k}, \rho_{k}\right| \geq c+1$. So for all $k$ not contained in the (open) intervals $\left(i_{h}, j_{h}\right)$ we have $\left|\chi_{k}, \rho_{k}\right| \geq c+1$. 
Put for a moment $j_{0}=i, i_{q+1}=j$. By the previous paragraph, by Lemma 9.11 and by the last assertion of Lemma 12.4, for any $0 \leq h \leq q$ and all $j_{h} \leq k \leq i_{h+1}$, either $\rho_{k}$ lies always between $\chi_{k}$ and $v_{k}$, or $\rho_{k}$ lies always between $\chi_{k}$ and $w_{k}$.

Now let us analyze what happens for a fixed $1 \leq h \leq q$ for $i_{h} \leq k \leq j_{h}$. Apply our argument under the additional assumption $p_{k}=p_{k}^{\prime}$ to $i=i_{h}, j=j_{h}$. Observe that if $\left|\chi_{i_{h}}, \rho_{i_{h}}\right| \geq c+1$ (which holds unless possibly $h=1$ and $i_{1}=i$ ) and $\chi_{i_{h}}$ lies between $\rho_{i_{h}}$ and $v_{i_{h}}$, then we have that $l=m=i_{h}$ (otherwise we have recorded that $\left|\rho_{k}, \chi_{k}\right| \leq c$ for $l \leq k \leq m$ ). Similarly, if $\left|\chi_{j_{h}}, \rho_{j_{h}}\right| \geq c+1$ (which holds unless possibly $h=q$ and $j_{q}=j$ ) and $\chi_{j_{h}}$ lies between $\rho_{j_{h}}$ and $w_{j_{h}}$, then $l=m=j_{h}$. In particular, those two situations cannot happen simultaneously, and if any of them happens, then either assertion (i) or assertion (iii) is valid for all $i_{h} \leq k \leq j_{h}$.

Summarizing, there can be at most one $h$ such that $l \neq j_{h}$ and $m \neq i_{h}$. If there is no such $h$, then either assertion (i) or assertion (iii) holds for all $i \leq k \leq j$ and we are done. If not, define $l, m$ as in the previous argument for $i=i_{h}, j=j_{h}$. They satisfy assertions (i)-(iii), as required.

Finally, we prove the last assertion. Pick $\lambda, l, m$ as above. Let $\gamma^{\prime}$ be the CAT(0) diagonal of the characteristic disc $\Delta$ for $(i-1, j+1)$. Since the maximal thickness for $\left(\sigma_{k}\right),\left(\tau_{k}\right)$ of the layers from $i$ to $j$ is $\geq 2 c+4$, then by Lemma 9.16(i)-(ii), we have that $v_{j+1}^{x}-w_{i-1}^{x} \geq 2 c+1$. Thus we can assume without loss of generality that $\lambda^{x}-w_{i-1}^{x} \geq c+\frac{1}{2}$. Thus $\lambda^{x}-\left(\gamma^{\prime} \cap v_{i} w_{i}\right)^{x} \geq c+\frac{1}{2}$ and $l>i$. Observe that $\lambda$ goes through the barycenter of $\chi_{l}$, hence $w_{l-1}^{x} \geq \lambda^{x}-\frac{1}{2}$ so $w_{l-1}^{x}-w_{i-1}^{x} \geq c$, as desired.

The next lemma in particular guarantees that in a "thick" interval, the vertices $\mathcal{S}_{p r}^{-1}\left(r_{k}\right)$ for $k$ in the "final" subinterval outside the "postfinal" subinterval form a coarse vertical line. We consider it, together with the previous lemma, the heart of the proof of Proposition 12.1. Below we put $\Delta$ to be the characteristic disc for the thick interval containing $i, j$ for $\left(\sigma_{k}\right),\left(\tau_{k}\right)$. Let $v_{k}, w_{k}$ be its boundary vertices, etc.

Lemma 12.9 Suppose that for some $i \leq j$ and for all $i \leq k \leq j$ the layer $k$ is thick for $\left(\sigma_{k}\right),\left(\tau_{k}\right),\left|p_{k}, \delta_{k}\right| \geq c+2 \geq 7$ and $\chi_{k}$ is either $\partial$-left or $c$-left from $\rho_{k}$. If $\left(\gamma^{\prime} \cap v_{j+1} w_{j+1}\right)^{x}=v_{j+1}^{x}+\frac{1}{2}$, then $v_{j+1}^{x}-v_{i}^{x}<c$.

Proof By contradiction. Roughly, the idea is the following. If $v_{j+1}^{x}$ is relatively large with respect to $v_{i}^{x}$, this means that the directed geodesic $\left(\sigma_{k}\right)$ performs in the layers $i, \ldots, j$ an unexpected turn towards $\left(\tau_{k}\right)$. On the other hand, there is plenty of room in the partial characteristic disc $\Delta_{p t}$ for $\left(p_{k}\right),\left(\tau_{k}\right)$, since $p_{k}$ are far away from $\delta_{k}$, hence (as we will see) away from $\sigma_{k}$. By assumption on $\chi_{k}$ the corresponding characteristic 
image $\mathcal{S}_{p t}\left(\Delta_{p t}\right)$ almost passes through $\sigma_{k}$. We can then see through $\Delta_{p t}$ that $\left(\sigma_{k}\right)$ actually goes vertically for all consecutive $i \leq k \leq j$. This yields a contradiction.

Formally, suppose $v_{j+1}^{x}-v_{i}^{x} \geq c$. By increasing $i$, if necessary, we may assume that $i$ is maximal $\leq j$ satisfying $v_{j+1}^{x}-v_{i}^{x} \geq c$. Hence $v_{j+1}^{x}-v_{i}^{x}=c$.

We claim that for all $i \leq k \leq j$ we have that $\chi_{k}$ is $\partial$-left. Indeed, by maximality of $i$ we have $\left(\gamma^{\prime} \cap v_{j+1} w_{j+1}\right)^{x}-v_{k}^{x} \leq c+\frac{1}{2}$. By Lemma 9.17 we have that $\left(\gamma^{\prime} \cap v_{k} w_{k}\right)^{x}-$ $\left(\gamma^{\prime} \cap v_{j+1} w_{j+1}\right)^{x}<0$. Putting these inequalities together implies that $\left|v_{k}, \rho_{k}\right| \leq c$. Hence if $\chi_{k}$ is $c$-left from $\rho_{k}$, then it equals $v_{k}$, thus it is also $\partial$-left, as required. Thus we have proved the claim. Moreover, $\left|v_{k}, \rho_{k}\right| \leq c$ together with $\left|p_{k}, \delta_{k}\right| \geq c+2$ gives also that $\left|p_{k}, \sigma_{k}\right| \geq 2$ and $p_{k} \neq t_{k}^{\prime}$ for $i \leq k \leq j$.

Denote $h_{k}=\mathcal{S}^{-1}\left(t_{k}^{\prime}\right) \in \chi_{k}$. By the claim we have $\left|v_{k} h_{k}\right| \leq 1$. Let $\Delta_{p t}$ be the characteristic disc for the thick interval $\left(i_{p t}, j_{p t}\right)$ for $\left(p_{k}\right),\left(t_{k}\right)$ containing $i \leq k \leq j$ and let $\mathcal{S}_{p t}$ be the corresponding characteristic mapping (we have $\left|p_{k} t_{k}\right|=\left|p_{k} t_{k}^{\prime}\right|+\left|t_{k}^{\prime} t_{k}\right| \geq 2$, since $\chi_{k}$ is $\partial$-left). Denote $\widetilde{v}_{k}=\mathcal{S}_{p t}^{-1}\left(p_{k}\right), \widetilde{w}_{k}=\mathcal{S}_{p t}^{-1}\left(t_{k}\right)$. Let $\widetilde{h}_{k}=\mathcal{S}_{p t}^{-1}\left(t_{k}^{\prime}\right)$. Since for $i \leq k \leq j$ we have $\left|t_{k} t_{k}^{\prime}\right| \geq 1$, by Lemma 12.6 the differences within $h_{k}^{x}$ (coordinates in $\Delta$ ) and within $\tilde{h}_{k}^{x}$ (coordinates in $\Delta_{p t}$ ) agree.

Now observe that $t_{k}^{\prime}$ spans a simplex with $\sigma_{k}$ by the claim, Lemma 10.3 and Lemma 9.8(iii)-(iv), for all $i \leq k \leq j$. Denote $\phi=\operatorname{span}\left\{t_{i}^{\prime}, \sigma_{i}\right\}$. Denote by $\phi_{i}=\phi, \phi_{i+1}, \ldots$ the simplices of the directed geodesic from $\phi$ to $\tau$. Denote by $\beta_{k}$ the simplices of the directed geodesic from $t_{i}^{\prime}$ to $\tau$. By Lemma 2.10 we have $\beta_{k} \subset \phi_{k} \supset \sigma_{k}$ for $k-i$ even, and $\beta_{k} \supset \phi_{k} \subset \sigma_{k}$ for $k-i$ odd. Denote by $\alpha_{k}$ the simplices of the directed geodesic in $\Delta_{p t}$ from $\widetilde{h}_{i}$ to $\widetilde{v}_{j_{p t}} \widetilde{w}_{j_{p t}}$.

First we prove that for all $i \leq k \leq j$ we have $\widetilde{v}_{k} \notin \alpha_{k}$. For $k=i$ this follows from $p_{i} \neq t_{i}^{\prime}$. For $k>i$ we argue by contradiction. Let $i<k_{0} \leq j$ be minimal such that $\widetilde{v}_{k_{0}} \in \alpha_{k_{0}}$. Observe that $\Delta_{p t}$ is actually a partial characteristic disc for $\left(p_{k}\right),\left(\tau_{k}\right)$ and $\left(\tau_{k}\right)$ is the directed geodesic from $\tau$ to $\sigma$. Hence, similarly as in Lemma 10.6, for $i \leq k \leq k_{0}$ the simplices $\alpha_{k}$ are alternatingly vertices and edges, with barycenters on a common vertical line. Moreover, by minimality of $k_{0}$, we have that $\alpha_{k_{0}}$ is an edge. By Lemma 10.5 and Lemma 2.10 (applied alternatingly for consecutive layers exactly as in the proof of Proposition 10.2), we have that $\beta_{k} \subset \mathcal{S}_{p t}\left(\alpha_{k}\right)$ for $k-i$ even and $\mathcal{S}_{p t}\left(\alpha_{k}\right) \subset \beta_{k}$ for $k-i$ odd, for all $i \leq k \leq k_{0}$. In particular, since $\alpha_{i}$ is a vertex and $\alpha_{k_{0}}$ is an edge, we have that $p_{k_{0}} \in \mathcal{S}_{p t}\left(\alpha_{k_{0}}\right) \subset \beta_{k_{0}} \supset \phi_{k_{0}} \subset \sigma_{k_{0}}$. But this contradicts $\left|p_{k_{0}}, \sigma_{k_{0}}\right| \geq 2$. Hence we proved that for all $i \leq k \leq j$ we have $\tilde{v}_{k} \notin \alpha_{k}$.

From the above proof we also get that for all $i \leq k \leq j$ we have $\beta_{k} \subset \mathcal{S}_{p t}\left(\alpha_{k}\right)$ for $k-i$ even and $\mathcal{S}_{p t}\left(\alpha_{k}\right) \subset \beta_{k}$ for $k-i$ odd, and the simplices $\alpha_{k}$ are alternatingly vertices and edges, with barycenters on a common vertical line. Since $t_{k}^{\prime}$ and $\sigma_{k}$ span 
a simplex, this implies that $t_{k}^{\prime} \in B_{2}\left(\mathcal{S}_{p t}\left(\alpha_{k}\right)\right)$, hence $\tilde{h}_{k} \in B_{2}\left(\alpha_{k}\right)$, for $i \leq k \leq j$. Since the barycenters of $\alpha_{k}$ lie on a common vertical line through $\widetilde{h}_{i}$, we conclude that $\left|\widetilde{h}_{i}^{x}-\widetilde{h}_{k}^{x}\right| \leq 2 \frac{1}{2}$ for $i \leq k \leq j$, in particular for $k=j$. But $\widetilde{h}_{j}^{x}-\widetilde{h}_{i}^{x}=h_{j}^{x}-h_{i}^{x} \geq c-1 \frac{1}{2}$. This contradicts $c \geq 5$.

We immediately get the following corollary, which excludes the possibility of adjoining a non-"thin" "final" subinterval of a "thick" interval to the beginning of a thin layer for $\left(\sigma_{k}\right),\left(\tau_{k}\right)$.

Corollary 12.10 Suppose that for some $i \leq j$ the layer $j+1$ is thin for $\left(\sigma_{k}\right),\left(\tau_{k}\right)$, and for all $i \leq k \leq j$ the layer $k$ is thick for $\left(\sigma_{k}\right),\left(\tau_{k}\right),\left|p_{k}, \delta_{k}\right| \geq c+2 \geq 7$ and $\chi_{k}$ is either $\partial$-left or $c$-left from $\rho_{k}$. Then $v_{j+1}^{x}-v_{i}^{x}<c$.

The next preparatory lemma takes care of the "thin" intervals for $\left(\sigma_{k}\right),\left(\tau_{k}\right)$. Let $d$ be a positive integer.

Lemma 12.11 Suppose that for some $i \leq j$ the layers $i, j$ for $\left(\sigma_{k}\right),\left(\tau_{k}\right)$ have thickness at most $d$ and for all $i \leq k \leq j$ the layer $k$ for $\left(\sigma_{k}\right),\left(\tau_{k}\right)$ has thickness at most $2 c+3$ and $\left|p_{k}, \delta_{k}\right| \geq 2 c+4$. Then the differences within $\left(\mathcal{S}_{p r}^{-1}\left(r_{k}\right)\right)^{x}$ are at most $c+2 d+2 \frac{1}{2}$.

We can also obtain an estimate independent of $c$ on the differences within $\left(\mathcal{S}_{p r}^{-1}\left(r_{k}\right)\right)^{x}$. However, we will not need it.

Proof We can define $p_{k}^{\prime}$ as usual (even for thin layers). Observe that we have $p_{k} \neq p_{k}^{\prime},\left|p_{k}, \sigma_{k}\right| \geq 2$, and $\left|p_{k}, \tau_{k}\right| \geq 2$, for $i \leq k \leq j$. Let $\tilde{s}_{k} \in \sigma_{k}, \tilde{t}_{k} \in \tau_{k}$ realize maximal distances from $p_{k}$ to $\sigma_{k}, \tau_{k}$, respectively. Let $\Delta_{p s}, \Delta_{p t}, \mathcal{S}_{p s}, \mathcal{S}_{p t}$ denote the characteristic discs and mappings for $\left(p_{k}\right),\left(\sigma_{k}\right)$ and $\left(p_{k}\right),\left(\tau_{k}\right)$, respectively, for the thick intervals containing all $i \leq k \leq j$. Since $p_{k} \neq p_{k}^{\prime}$, we have by Lemma 12.6 that the differences within $\left(\mathcal{S}_{p s}^{-1}\left(p_{k}\right)\right)^{x}$, within $\left(\mathcal{S}_{p t}^{-1}\left(p_{k}\right)\right)^{x}$, and within $\left(\mathcal{S}_{p r}^{-1}\left(p_{k}\right)\right)^{x}$ agree, if we vary $k$ among $i \leq k \leq j$.

For $i \leq k \leq j$ denote $\dot{s}_{k}=\mathcal{S}_{p s}^{-1}\left(\widetilde{s}_{k}\right), \dot{t}_{k}=\mathcal{S}_{p t}^{-1}\left(\widetilde{t}_{k}\right)$. Let $i \leq k_{1}<k_{2} \leq j$. By Lemma 9.16(i)-(ii) we have that $\dot{s}_{k_{1}}^{x}-\dot{s}_{k_{2}}^{x} \geq-\frac{1}{2}$ and $\dot{t}_{k_{1}}^{x}-\dot{t}_{k_{2}}^{x} \leq \frac{1}{2}$. In particular, $\dot{s}_{k_{2}}^{x}-\dot{s}_{j}^{x} \geq-\frac{1}{2}$ and $\dot{s}_{i}^{x}-\dot{s}_{k_{1}}^{x} \geq-\frac{1}{2}$, for $i \leq k_{1}<k_{2} \leq j$. Hence

$$
\dot{s}_{k_{2}}^{x}-\dot{s}_{k_{1}}^{x} \geq \dot{s}_{j}^{x}-\dot{s}_{i}^{x}-1 \geq \dot{t}_{j}^{x}-\dot{t}_{i}^{x}-1-2 d \geq-2 d-1 \frac{1}{2} .
$$

Analogously

$$
\dot{t}_{k_{2}}^{x}-\dot{t}_{k_{1}}^{x} \leq 2 d+1 \frac{1}{2} \text {. }
$$


It will be convenient for us to assume that the coordinates in $\Delta_{p s}, \Delta_{p t}$ agree on $\mathcal{S}_{p s}^{-1}\left(p_{k}\right)$ and $\mathcal{S}_{p t}^{-1}\left(p_{k}\right)$, so that we can compare coordinates of points in $\Delta_{p s}$ and $\Delta_{p t}$. With this convention, for any $i \leq k_{1}, k_{2} \leq j$ we have that $\dot{s}_{k_{1}}^{x}-\dot{t}_{k_{2}}^{x} \geq \dot{s}_{j}^{x}-\dot{t}_{j}^{x}-1 \geq$ $-d-1$. Analogously $\dot{s}_{k_{1}}^{x}-\dot{t}_{k_{2}}^{x} \leq d+1$. So altogether the differences within all the $\dot{s}_{k}^{x}, \dot{t}_{k}^{x}$, where $i \leq k \leq j$, are at most $2 d+1 \frac{1}{2}$. In particular, if we denote by $a$ the minimum over $k$ of $\dot{s}_{k}^{x}, \dot{t}_{k}^{x}$ and by $b$ the maximum over $k$ of $\dot{s}_{k}^{x}, \dot{t}_{k}^{x}$, we get $b-a \leq 2 d+1 \frac{1}{2}$.

For a fixed $k$, since the thickness of the layer $k$ is at most $2 c+3$, we have that $\left|\tilde{s}_{k} r_{k}\right| \leq c+1$ or $\left|\tilde{t}_{k} r_{k}\right| \leq c+1$, hence

$$
\min \left\{\left|p_{k} \widetilde{s}_{k}\right|,\left|p_{k} \tilde{t}_{k}\right|\right\} \leq\left|p_{k} r_{k}\right|+c+1,
$$

thus $r_{k}^{x} \geq a-(c+1)$. On the other hand, by convexity of balls we have

$$
\left|p_{k} r_{k}\right| \leq \max \left\{\left|p_{k} \tilde{s}_{k}\right|,\left|p_{k} \tilde{t}_{k}\right|\right\},
$$

hence $r_{k}^{x} \leq b$. Altogether, this implies that the differences within $\left(\mathcal{S}_{p r}^{-1}\left(r_{k}\right)\right)^{x}$ are at most

$$
(c+1)+\left(2 d+1 \frac{1}{2}\right)+1=c+2 d+2 \frac{1}{2} .
$$

Finally, we prove the following easy lemma, which is needed both here and later in Section 13.

Lemma 12.12 Let $\Delta$ be a generalized characteristic disc for $(i, j)$. Let $\gamma$ be a CAT(0) geodesic in $\Delta$ connecting some points in $v_{i} w_{i}, v_{j} w_{j}$. For $i \leq k \leq j$ let $h_{k} \in v_{k} w_{k}$ be some points at distance $\leq \frac{1}{2}$ from $\gamma \cap v_{k} w_{k}$. Let $\Delta$ split $\subset \Delta$ be the generalized characteristic disc for $(i, j)$ with $w_{k}$ substituted with $h_{k}$. Then the CAT(0) geodesic $h_{i} h_{j}$ in $\Delta$ split is 1 -close to the piecewise linear boundary path $h_{i} h_{i+1} \cdots h_{j}$.

Proof For $i \leq k \leq j$ let $h_{k}^{\prime}$ be the points on $v_{k} w_{k}$ with $\left(h_{k}^{\prime}\right)^{x}=\max \left\{\left(\gamma \cap v_{k} w_{k}\right)^{x}, h_{k}^{x}\right\}$. Let $\Delta_{\text {cut }} \subset \Delta$ be the generalized characteristic disc for $(i, j)$ with $w_{k}$ substituted with $h_{k}^{\prime}$. Then $\gamma$ is also a CAT(0) geodesic in $\Delta_{\text {cut }}$. By Lemma 11.7 applied to $\Delta$ split $\subset \Delta_{\text {cut }}$ we have that the $\operatorname{CAT}(0)$ geodesic $h_{i} h_{j}$ in $\Delta$ split is $\frac{1}{2}$-close to $\gamma$, hence 1 -close to the path $h_{i} h_{i+1} \cdots h_{j}$.

Now we are ready to put together all pieces of the puzzle.

Proof of Proposition 12.1 Put $c=5$. For the layers $k$ such that $\left|p_{k} r_{k}\right| \leq 7 c+12$ there is nothing to prove. Now suppose that for some $i^{\prime}<j^{\prime}$, where $j^{\prime}-i^{\prime} \geq 2$, we 
have $\left|p_{i^{\prime}} r_{i^{\prime}}\right|=\left|p_{j^{\prime}} r_{j^{\prime}}\right|=7 c+12$ and for $i^{\prime}<k<j^{\prime}$ we have $\left|p_{k} r_{k}\right| \geq 7 c+13$, hence $\left|p_{k}, \delta_{k}\right| \geq 7 c+12$. In particular, $p_{k}$ are as far from $\delta_{k}$ as required in Lemma 12.9 and Corollary 12.10 .

Let $\Delta_{p r}$ be the partial characteristic disc for $\left(i^{\prime}, j^{\prime}\right)$ for $\left(p_{k}\right),\left(r_{k}\right)$, and let $\mathcal{S}_{p r}$ be the corresponding partial characteristic mapping. Denote $u_{k}=\mathcal{S}_{p r}^{-1}\left(r_{k}\right)$.

Step 1 There exist $i^{\prime} \leq l \leq m \leq j^{\prime}$ such that

(1) for $i^{\prime} \leq k<l$ the layer $k$ is thick for $\left(\sigma_{t}\right),\left(\tau_{t}\right)$, every 1 -skeleton geodesic $p_{k} s_{k}$ intersects $\delta_{k}$, and $\left(\gamma^{\prime} \cap v_{k} w_{k}\right)^{x}<w_{k}^{x}-\frac{1}{2}$ (in the appropriate characteristic disc for $\left(\sigma_{t}\right),\left(\tau_{t}\right)$, with the usual notation $v_{k}, w_{k}$, etc.),

(2) among $l \leq k \leq m$ the differences within $u_{k}^{x}$ are at most $7 c+10 \frac{1}{2}$,

(3) for $j^{\prime} \geq k>m$ the layer $k$ is thick for $\left(\sigma_{t}\right),\left(\tau_{t}\right)$, every 1 -skeleton geodesic $p_{k} t_{k}$ intersects $\delta_{k}$, and $\left(\gamma^{\prime} \cap v_{k} w_{k}\right)^{x}>v_{k}^{x}+\frac{1}{2}$.

This is the division into the "preinitial" interval, the union of the central intervals, and the "postfinal" interval in the language of the outline of the proof.

Let us justify Step 1. First consider the simple case that there are no thin layers for $\left(\sigma_{k}\right),\left(\tau_{k}\right)$ among the layers $i^{\prime} \leq k \leq j^{\prime}$. Then Lemma 12.8 applied to $i=i^{\prime}, j=j^{\prime}$ gives us a pair of numbers $l^{\prime}, m^{\prime}$, which satisfies assertions (1) and (3) of Step 1 (with $l^{\prime}, m^{\prime}$ in place of $l, m$ ), except for the statements on the position of $\gamma^{\prime}$ (we will refer to these as incomplete assertions (1) and (3)).

Let $l<l^{\prime}$ be minimal $\geq i^{\prime}$ such that $\left(\gamma^{\prime} \cap v_{l} w_{l}\right)^{x}=w_{l}^{x}-\frac{1}{2}$ (if there is no such $l$, in particular, if $l^{\prime}=i^{\prime}$, then we put $l=l^{\prime}$ ). Similarly, let $m>m^{\prime}$ be maximal $\leq j^{\prime}$ such that $\left(\gamma^{\prime} \cap v_{m} w_{m}\right)^{x}=v_{m}^{x}+\frac{1}{2}$ (if there is no such $m$, in particular, if $m^{\prime}=j^{\prime}$, then we put $m=m^{\prime}$ ). Obviously, $l, m$ satisfy complete assertions (1) and (3) of Step 1. To prove that they satisfy assertion (2), we need the following.

Claim Among $l \leq k \leq l^{\prime}-1$ the differences within $u_{k}^{x}$ are at most $c+1$. Analogously, among $m^{\prime}+1 \leq k \leq m$ the differences within $u_{k}^{x}$ are at most $c+1$.

To justify the claim, we need to introduce some notation. Up to the end of the proof of the claim we consider $l \leq k \leq l^{\prime}-1$. Observe that the layers $k$ for $\left(p_{k}\right),\left(s_{k}\right)$ are thick, since by incomplete assertion (1) we have that $\left|p_{k} s_{k}\right|>\left|p_{k}, \delta_{k}\right|$. Denote by $\Delta, \mathcal{S}$ (resp. $\Delta_{p s}, \mathcal{S}_{p s}$ ) the characteristic disc and mapping for the thick interval containing $k$ for $\left(\sigma_{t}\right),\left(\tau_{t}\right)$ (resp. for $\left.\left(p_{t}\right),\left(s_{t}\right)\right)$. For each $k$ let $\bar{h}_{k}$ be the vertex in $\delta_{k} \cap p_{k} s_{k}$ closest to $p_{k}$ (for some 1-skeleton geodesic $p_{k} s_{k}$ ). By Proposition 7.6 we have that $\bar{h}_{k} \in \mathcal{S}_{p s}\left(\Delta_{p s}\right)$. Denote $h_{k}=\mathcal{S}^{-1}\left(\bar{h}_{k}\right), \widetilde{h}_{k}=\mathcal{S}_{p s}^{-1}\left(\bar{h}_{k}\right)$. Since by 
incomplete assertion (1) we have $s_{k} \neq s_{k}^{\prime}$, Lemma 12.6 gives that the differences within $-h_{k}^{x}$ and within $\widetilde{h}_{k}^{x}$ agree (the sign changes since $\left(s_{k}\right)$ plays the role of the left boundary component in $\mathcal{S}(\Delta)$ and the right one in $\mathcal{S}_{p s}\left(\Delta_{p s}\right)$ ). By Lemma 12.6 applied to $\Delta_{p s}$ and $\Delta_{p r}$, and since $\left|p_{k} r_{k}\right|=\left|p_{k} \bar{h}_{k}\right|$ or $\left|p_{k} r_{k}\right|=\left|p_{k} \bar{h}_{k}\right|+1$, we have that the differences within $u_{k}^{x}$ differ at most by 1 from the differences within $\tilde{h}_{k}^{x}$. Hence the differences within $u_{k}^{x}$ differ at most by 1 from the differences within $-h_{k}^{x}$.

Now we can proceed with justifying the claim. By Lemma 12.9 we have $w_{l^{\prime}-1}^{x}-w_{l}^{x}<$ $c$, hence $\left(\gamma^{\prime} \cap v_{l} w_{l}\right)^{x} \geq w_{l^{\prime}-1}^{x}-c$. Thus, by Lemma 9.17, we have $\left(\gamma^{\prime} \cap v_{k} w_{k}\right)^{x} \geq$ $w_{l^{\prime}-1}^{x}-c$ for all $k$. This implies, by the definition of $\rho_{k}$, that $h_{k}^{x} \geq w_{l^{\prime}-1}^{x}-c-\frac{1}{2}$. On the other hand, by Lemma 9.16 we have that $w_{k}^{x} \leq w_{l^{\prime}-1}^{x}+\frac{1}{2}$, hence we have $h_{k}^{x} \leq w_{l^{\prime}-1}^{x}-\frac{1}{2}$. Thus the differences within $h_{k}^{x}$ are at most $c$, hence the differences within $u_{k}^{x}$ are at most $c+1$. This justifies the first assertion of the claim. The second one is proved analogously.

Now we can finish the proof of Step 1 in the simple case that there are no thin layers for $\left(\sigma_{k}\right),\left(\tau_{k}\right)$, among the layers $i^{\prime} \leq k \leq j^{\prime}$. To prove assertion (2), we need to compare $u_{k_{1}}^{x}$ and $u_{k_{2}}^{x}$, for $l \leq k_{1}<k_{2} \leq m$. Assume, which is the worst possible case, that $l \leq k_{1} \leq l^{\prime}-1$ and $m^{\prime}+1 \leq k_{2} \leq m$. By Lemma 12.8(ii) and by the claim we have

$$
\begin{aligned}
\left|u_{k_{1}}^{x}-u_{k_{2}}^{x}\right| & \leq\left|u_{k_{1}}^{x}-u_{l^{\prime}-1}^{x}\right|+\frac{1}{2}+\left|u_{l^{\prime}}^{x}-u_{m^{\prime}}^{x}\right|+\frac{1}{2}+\left|u_{m^{\prime}+1}^{x}-u_{k_{2}}^{x}\right| \\
& \leq(c+1)+\frac{1}{2}+(c+1)+\frac{1}{2}+(c+1),
\end{aligned}
$$

which is even better then the required estimate. This ends the proof of Step 1 in the simple case.

Now consider the complex case that there is a thin layer among the layers $i^{\prime} \leq k \leq j^{\prime}$. Let $\left(l_{0}, m_{0}\right)$ be a maximal (with respect to inclusion) interval, with $i^{\prime} \leq l_{0} \leq m_{0} \leq j^{\prime}$, such that the layers $l_{0}, m_{0}$ are thin for $\left(\sigma_{k}\right),\left(\tau_{k}\right)$ and for $l_{0}<k<m_{0}$ the layer $k$ has thickness at most $2 c+3$ (possibly $l_{0}=m_{0}$ ). This is the "proper thin" interval of the outline of the proof.

First we argue that for $i^{\prime} \leq k<l_{0}$ and $m_{0}<k \leq j^{\prime}$ the layer $k$ is thick. Otherwise, suppose without loss of generality that $k_{0}$ is maximal $<l_{0}$ such that the layer $k_{0}$ is thin. Then, by maximality of $\left(l_{0}, m_{0}\right)$, the thick interval $\left(k_{0}, l_{0}\right)$ contains some $k$ such that the layer $k$ has thickness at least $2 c+4$. Thus by the last assertion of Lemma 12.8 applied to $i=k_{0}+1, j=l_{0}-1$ we get $k_{0}<l \leq m<l_{0}$ so that either $m<l_{0}-1$ and $v_{l_{0}}^{x}-v_{m+1}^{x} \geq c$, or $l>k_{0}+1$ and $w_{l-1}^{x}-w_{k_{0}}^{x} \geq c$. In both cases this contradicts Corollary 12.10 applied respectively to $i=m+1, j=l_{0}-1$, or to $i=l-1, j=k_{0}+1$ with the roles of $v, w$ interchanged and the order on naturals 
inversed. Thus we have proved that for $i^{\prime} \leq k<l_{0}$ and $m_{0}<k \leq j^{\prime}$ the layer $k$ is thick for $\left(\sigma_{k}\right),\left(\tau_{k}\right)$.

Now we can apply Lemma 12.8 to $i=i^{\prime}, j=l_{0}-1$. Denote by $l^{\prime}, m^{\prime}$ the pair of numbers given by its assertion. By Corollary 12.10 we have that $v_{l_{0}}^{x}-v_{k}^{x}<c$ for $m^{\prime}+1 \leq k \leq l_{0}$. Similarly, we apply Lemma 12.8 to $i=m_{0}+1, j=j^{\prime}$ and denote by $l^{\prime \prime}, m^{\prime \prime}$ the pair of numbers given by its assertion. By Corollary 12.10 we have $w_{k}^{x}-w_{m_{0}}^{x}<c$ for $m_{0} \leq k \leq l^{\prime \prime}-1$. Hence, by Lemma 9.16(i)-(ii), the thickness of the layer $k$, for $m^{\prime}+1 \leq k \leq l_{0}$ and for $m_{0} \leq k \leq l^{\prime}-1$, is at most $c+1$.

Define, similarly as before, $l<l^{\prime}$ to be minimal $\geq i^{\prime}$ such that $\left(\gamma^{\prime} \cap v_{l} w_{l}\right)^{x}=w_{l}^{x}-\frac{1}{2}$ (if there is no such $l$, in particular, if $l^{\prime}=i^{\prime}$, then we put $l=l^{\prime}$ ), in appropriate characteristic disc. Similarly, let $m>m^{\prime \prime}$ be maximal $\leq j^{\prime}$ such that $\left(\gamma^{\prime} \cap v_{m} w_{m}\right)^{x}=$ $v_{m}^{x}+\frac{1}{2}$ (if there is no such $m$, in particular, if $m^{\prime \prime}=j^{\prime}$, then we put $m=m^{\prime \prime}$ ).

For $l, m$ as above we have that assertion (1) follows from Lemma 12.8(i) and assertion (3) follows from Lemma 12.8(iii). As for assertion (2), assume, which is the worst possible case, that $l \leq k_{1} \leq l^{\prime}-1$ and $m^{\prime \prime}+1 \leq k_{2} \leq m$. Combining Lemma 12.11 applied to $i=m^{\prime}+1, j=l^{\prime \prime}-1, d=c+1$ with Lemma 12.8(ii) and with the claim above (which is also valid in this complex case) we get

$$
\begin{aligned}
\left|u_{k_{1}}^{x}-u_{k_{2}}^{x}\right| \leq & \left|u_{k_{1}}^{x}-u_{l^{\prime}-1}^{x}\right|+\frac{1}{2}+\left|u_{l^{\prime}}^{x}-u_{m^{\prime}}^{x}\right|+\frac{1}{2}+\left|u_{m^{\prime}+1}^{x}-u_{l^{\prime \prime}-1}^{x}\right| \\
& +\frac{1}{2}+\left|u_{l^{\prime \prime}}^{x}-u_{m^{\prime \prime}}^{x}\right|+\frac{1}{2}+\left|u_{m^{\prime \prime}+1}^{x}-u_{k_{2}}^{x}\right| \\
\leq & (c+1)+\frac{1}{2}+(c+1)+\frac{1}{2}+\left(c+2 d+2 \frac{1}{2}\right) \\
& +\frac{1}{2}+(c+1)+\frac{1}{2}+(c+1)=7 c+10 \frac{1}{2},
\end{aligned}
$$

as required. Thus we have completed the proof of Step 1.

Step $2 \gamma_{p r}$ is $97-$ close to $\left(u_{k}\right)$.

For the layers $i^{\prime} \leq k<l$ define $\Delta, \mathcal{S}, \Delta_{p s}, \mathcal{S}_{p s}$ and $h_{k} \in \rho_{k} \subset \Delta, \tilde{h}_{k} \in \Delta_{p s}, \bar{h}_{k}=$ $\mathcal{S}\left(h_{k}\right)=\mathcal{S}_{p s}\left(\tilde{h}_{k}\right)$ like in Step 1 (which is possible by assertion (1) of Step 1). Recall that the differences within $u_{k}^{x}$ differ at most by 1 from the differences within $-h_{k}^{x}$. In particular, since for $i^{\prime} \leq k_{1}<k_{2}<l$ we have $h_{k_{1}}^{x}-h_{k_{2}}^{x} \leq \frac{1}{2}$ (by Lemma 9.17 and the definition of $\rho_{k}$ ), it follows that $u_{k_{2}}^{x}-u_{k_{1}}^{x} \leq 1 \frac{1}{2}$. Analogously we choose vertices $h_{k} \in \rho_{k}$ (in appropriate characteristic disc) for $m<k \leq j^{\prime}$, so that $\left|p_{k} r_{k}\right|=\left|p_{k} \bar{h}_{k}\right|$ or $\left|p_{k} r_{k}\right|=\left|p_{k} \bar{h}_{k}\right|+1$. Hence for $m<k_{2}<k_{1} \leq j^{\prime}$ we have $u_{k_{2}}^{x}-u_{k_{1}}^{x} \leq 1 \frac{1}{2}$. 
Let $l \leq k_{0} \leq m$ be such that $u_{k_{0}}^{x}$ is minimal. Let $\alpha$ be a vertical line segment in $\Delta_{p r}$ from the layer $\max \left\{l-1, i^{\prime}+1\right\}$ to the layer $\min \left\{m+1, j^{\prime}-1\right\}$ at distance 2 to the left from $u_{k_{0}}$. By assertion (2) of Step 1 and by the fact that $\left|p_{k} r_{k}\right| \geq 7 c+13$ this line segment is really contained in $\Delta_{p r}$. Let $\beta_{1}, \beta_{2}$ be CAT(0) geodesics in $\Delta_{p r}$ connecting $u_{i^{\prime}}, u_{j^{\prime}}$ to the endpoints of $\alpha$. Since $u_{k_{2}}^{x}-u_{k_{1}}^{x} \leq 1 \frac{1}{2}$ for $i^{\prime} \leq k_{1}<k_{2}<l$ and $m<k_{2}<k_{1} \leq j^{\prime}$, we have for all $i^{\prime} \leq k \leq j^{\prime}$ that $u_{k}^{x}>\alpha^{x}$. Hence the region in $\Delta_{p r}$ to the right of the concatenation $\beta_{1} \alpha \beta_{2}^{-1}$ is convex, and thus contains the CAT(0) geodesic in $\Delta_{p r}$ joining $u_{i^{\prime}}$ with $u_{j^{\prime}}$.

We claim that $\beta_{1}$ is $(7 c+15)$-close to $\left(u_{k}\right)$. Indeed, if $l=i^{\prime}$ or $l-1=i^{\prime}$, then this is easy. Otherwise, let $i^{\prime} \leq k \leq l-1$. Let $\left.\Delta^{\prime \prime} \subset \Delta\right|_{i^{\prime}} ^{l-1}$ be the generalized characteristic disc for $\left(i^{\prime}, l-1\right)$ obtained from $\left.\Delta^{\prime}\right|_{i^{\prime}} ^{l-1}$ (the modified characteristic disc, in which $\gamma^{\prime}$ is a $\operatorname{CAT}(0)$ geodesic) by substituting $w_{k}^{\prime}$ with $w_{k}^{\prime \prime}$, such that $\left(w_{k}^{\prime \prime}\right)^{x}=h_{k}^{x}+1$. Denote $\gamma^{\prime}$ restricted to the layers from $i^{\prime}$ to $l-1$ by $\left.\gamma^{\prime}\right|_{i^{\prime}} ^{l-1}$. We have $\left.\gamma^{\prime}\right|_{i^{\prime}} ^{l-1} \subset \Delta^{\prime \prime}$ and by assertion (1) of Step 1 we have that $\left.\gamma^{\prime}\right|_{i^{\prime}} ^{l-1}$ does not touch the $\left(w_{k}^{\prime}\right)$ boundary component of $\Delta^{\prime}$, hence it is also in $\Delta^{\prime \prime}$ a CAT(0) geodesic (which does not touch $w_{k}^{\prime \prime}$ ). Let $\left.\Delta_{p s}^{\prime} \subset \Delta_{p s}\right|_{i^{\prime}} ^{l-1}$ be the generalized characteristic disc for $\left(i^{\prime}, l-1\right)$ obtained from $\left.\Delta_{p s}\right|_{i^{\prime}} ^{l-1}$ by deleting $\frac{1}{2}$-horizontal neighborhood of the boundary component corresponding to $\left(s_{k}\right)$. Observe that there is an (orientation reversing) embedding $e^{\prime \prime}: \Delta^{\prime \prime} \rightarrow \Delta_{p s}^{\prime}$, and that $e^{\prime \prime}\left(\gamma^{\prime}\right)$ is still a CAT(0) geodesic in $\Delta_{p s}^{\prime}$. Moreover, $e^{\prime \prime}\left(h_{k}\right)=\tilde{h}_{k}$, so that $\left|e^{\prime \prime}\left(\gamma^{\prime} \cap v_{k} w_{k}\right) \tilde{h}_{k}\right| \leq \frac{1}{2}$.

Let $\left.\Delta_{p h} \subset \Delta_{p s}\right|_{i^{\prime}} ^{l-1}$ be the generalized characteristic disc for $\left(i^{\prime}, l-1\right)$ obtained from $\Delta_{p s}^{\prime}$ by splitting along $\widetilde{h}_{k}$ (in fact $\bar{h}_{k}$ is a 1 -skeleton geodesic and $\Delta_{p h}$ is the partial characteristic disc for $\left(p_{k}\right),\left(\bar{h}_{k}\right)$, but we do not need this). By Lemma 12.12 the CAT $(0)$ geodesic $\widetilde{h}_{i^{\prime}} \widetilde{h}_{l-1}$ in $\Delta_{p h}$ is 1 -close to the boundary path $\left(\tilde{h}_{k}\right)$. Now recall that there is an embedding $e: \Delta_{p h} \rightarrow \Delta_{p r}$, such that $\left|e\left(\tilde{h}_{k}\right) u_{k}\right| \leq 1$. Let us compute the distances between the endpoints of the image under $e$ of the CAT(0) geodesic $\widetilde{h}_{i^{\prime}} \widetilde{h}_{l-1}$ and the endpoints of $\beta_{1}$ in $\Delta_{p r}$. The distance between $e\left(\widetilde{h}_{i^{\prime}}\right)$ and $u_{i^{\prime}}$ is at most 1 , and the distance between the second pair of endpoints is at most $2+\left(7 c+10 \frac{1}{2}\right)+\frac{1}{2}$ by assertion (2) of Step 1. Hence, by Lemma 11.7, we have that $e\left(\tilde{h}_{i}, \tilde{h}_{l-1}\right)$ is $(7 c+13)-$ close to $\beta_{1}$. Recall that $e\left(\tilde{h}_{i^{\prime}} \widetilde{h}_{l-1}\right)$ is $1-$ close to $e\left(\left(\tilde{h}_{k}\right)\right)$, which is 1 -close to $\left(u_{k}\right)$. Altogether, $\beta_{1}$ is $((7 c+13)+1+1)$-close to $\left(u_{k}\right)$, as desired. Thus we have justified the claim. Analogously, $\beta_{2}$ is $(7 c+15)-$ close to $\left(u_{k}\right)$.

From the claim and since, by assertion (2) of Step $1, \alpha$ is $(7 c+13)$-close to $\left(u_{k}\right)$, it follows that the two boundary components of the convex region in $\Delta_{p r}$ to the right of $\beta_{1} \alpha \beta_{2}^{-1}$ are $(7 c+15)$-close. Hence the CAT $(0)$ geodesic $u_{i^{\prime}} u_{j^{\prime}}$ in $\Delta_{p r}$ is $(7 c+15)$-close to $\left(u_{k}\right)$. Now consider the CAT $(0)$ geodesic $\gamma_{p r}$ in $\Delta_{p r}$ (which appears in the statement of the proposition) restricted to the layers from $i^{\prime}$ to $j^{\prime}$. Since 
its endpoints are at distance $\leq 7 c+12$ from the endpoints of $u_{i^{\prime}} u_{j^{\prime}}$ (this is because $\left|p_{i^{\prime}} r_{i^{\prime}}\right|=7 c+12=\left|p_{j^{\prime}} r_{j^{\prime}}\right|$ ), we get (by Lemma 11.7, we do not vary the boundary this time) that $\gamma_{p r}$ is $(14 c+27)-$ close to $u_{k}$, as desired (recall that $c=5$ ).

\section{Contracting}

In this section we prove the following consequence of Proposition 12.1, which summarizes the contracting properties of Euclidean geodesics.

Theorem 13.1 (Theorem C) Let $s, s^{\prime}, t$ be vertices in a systolic complex $X$ such that $|s t|=n,\left|s^{\prime} t\right|=n^{\prime}$. Let $\left(r_{k}\right)_{k=0}^{n},\left(r_{k}^{\prime}\right)_{k=0}^{n^{\prime}}$ be 1-skeleton geodesics such that $r_{k} \in$ $\delta_{k}, r_{k}^{\prime} \in \delta_{k}^{\prime}$, where $\left(\delta_{k}\right),\left(\delta_{k}^{\prime}\right)$ are Euclidean geodesics for $t, s$ and for $t, s^{\prime}$ respectively. Then for all $0 \leq c \leq 1$ we have $\left|r_{\lfloor c n\rfloor} r_{\left\lfloor c n^{\prime}\right\rfloor}^{\prime}\right| \leq c\left|s s^{\prime}\right|+C$, where $C$ is a universal constant.

In the proof we need three easy preparatory lemmas.

Lemma 13.2 Let $D$ be a 2-dimensional systolic complex (in particular CAT(0) with the standard piecewise Euclidean metric). Let $x, y$ be vertices in $D$. Then there exists a 1 -skeleton geodesic $\omega$ in $D$ joining $x, y$ such that if $D_{0}$ is the union with $\omega$ of a connected component of $D \backslash \omega$, then the CAT( 0$)$ geodesic $x y$ in is $1-$ close to $\omega$ in $L \cap D_{0}$, where $L$ is the convex hull in $D$ of $x \cup y$.

Proof Let $L_{i}$ be the layers in $D$ between $x, y$. Then $L$ is the span in $D$ of the union of $L_{i}$. Observe that $L$ is convex in CAT(0) sense in $D$. Hence the CAT(0) geodesic $x y$ in $D$ is contained in $L$. Now similarly as in Definition 9.10 define vertices $\omega_{i} \in L_{i}$ to be the vertices nearest to $x y \cap L_{i}$ (possibly nonunique). Analogously as in Lemma 9.11 one proves that $\omega_{i}, \omega_{i+1}$ are neighbors, hence $\left(\omega_{i}\right)$ form a path $\omega$, which is a 1 -skeleton geodesic. By the construction we have $\left|\omega_{i}, x y \cap L_{i}\right| \leq \frac{1}{2}$ (here $|\cdot, \cdot \cdot|$ denotes the distance along the straight line). For a fixed $D_{0}$ the CAT $(0)$ geodesic $x y$ in $D_{0}$ is contained in the convex $L \cap D_{0}$, hence it is 1 -close to $\omega$ by Lemma 12.12 applied to $L$.

Lemma 13.3 Let $\Delta$ be a generalized characteristic disc for $(i, j)$. Let $\Delta$ split $\subset \Delta$ be a generalized characteristic disc for $(i, j)$ with $w_{k}$ substituted with $\dot{w}_{k}$ for some $\dot{w}_{k} \in v_{k} w_{k}$. Let $\gamma, \dot{\gamma}$ be CAT(0) geodesics with common endpoints in the layers $i, j$ in $\Delta, \Delta$ split, respectively. Then $\dot{\gamma} \cap v_{k} w_{k}$ is not farther from $v_{k}$ than $\gamma \cap v_{k} w_{k}$.

Proof Let $\Delta_{0} \subset \Delta$ be the characteristic disc for $(i, j)$ with $w_{k}$ substituted with $\gamma \cap v_{k} w_{k}$. Then $\Delta_{0} \cap \Delta$ split is convex in $\Delta$ split and we are done. 
Lemma 13.4 Let $T$ be a CAT(0) (ie simply connected) subspace of $\mathbb{E}^{2}$, whose boundary is an embedded loop which consists of three geodesic (in $T$ ) segments $\alpha, \beta, \gamma$, where $\alpha$ is contained in a straight line in $\mathbb{E}^{2}$. Denote $x=\beta \cap \gamma$. Let $\eta$ be a geodesic in $T$ contained in a straight line parallel to $\alpha$ with endpoints on $\beta, \gamma$. Let $c$ denote the ratio of the distances in $\mathbb{E}^{2}$ between $x$ and the line containing $\eta$ and between $x$ and the line containing $\alpha$. Then $|\eta| /|\alpha| \leq c$.

Proof Let $y_{1}, y_{2} \in \mathbb{E}^{2}$ be points on the line containing $\eta$ colinear with $x$ and the endpoints of $\alpha$. By the Tales Theorem we have $\left|y_{1} y_{2}\right| /|\alpha|=c$. On the other hand, since $\beta, \gamma$ are geodesics in $T$, we get that $\eta \subset y_{1} y_{2}$.

We are now ready for the endgame.

Proof of Theorem 13.1 (Theorem C) Let $m$ be maximal satisfying $r_{m}=r_{m}^{\prime}$. First assume that $\lfloor c n\rfloor \leq m$ or $\left\lfloor c n^{\prime}\right\rfloor \leq m$, say $\left\lfloor c n^{\prime}\right\rfloor \leq m$. Then $\left|r_{\left\lfloor c n^{\prime}\right\rfloor} r_{\left\lfloor c n^{\prime}\right\rfloor}^{\prime}\right| \leq 194$. Indeed, let $\Delta$ be the characteristic disc for $\left(r_{i}\right),\left(r_{i}^{\prime}\right)$ between $t$ and $r_{m}=r_{m}^{\prime}$, for the thick interval containing $\left\lfloor c n^{\prime}\right\rfloor$ (if layer $\left\lfloor c n^{\prime}\right\rfloor$ is thin then there is nothing to prove). Then by Proposition 12.1 applied to $\left(r_{i}\right)_{i=0}^{n}$ and $r_{0}^{\prime}, \ldots r_{m}^{\prime}, r_{m+1}, \ldots r_{n}$ we get that the CAT $(0)$ geodesic in $\Delta$ joining the barycenters of the two outermost edges is $97-$ close to the boundary component corresponding to $\left(r_{i}\right)$. Similarly we get that this CAT(0) geodesic is $97-$ close to the second boundary component. Altogether we get that $\left|r_{\left\lfloor c n^{\prime}\right\rfloor} r_{\left\lfloor c n^{\prime}\right\rfloor}^{\prime}\right| \leq 194$, as desired. This yields

$$
\begin{aligned}
\left|r_{\lfloor c n\rfloor} r_{\left\lfloor c n^{\prime}\right\rfloor}^{\prime}\right| & \leq\left|r_{\lfloor c n\rfloor} r_{\left\lfloor c n^{\prime}\right\rfloor}\right|+\left|r_{\left\lfloor c n^{\prime}\right\rfloor} r_{\left\lfloor c n^{\prime}\right\rfloor}^{\prime}\right| \leq\left|\lfloor c n\rfloor-\left\lfloor c n^{\prime}\right\rfloor\right|+194 \\
& <c\left|n-n^{\prime}\right|+195 \leq c\left|s s^{\prime}\right|+195,
\end{aligned}
$$

as required. So from now on we assume that $\lfloor c n\rfloor>m$ and $\left\lfloor c n^{\prime}\right\rfloor>m$.

Let $k$ be minimal such that $r_{k}$ lies on some 1 -skeleton geodesic $s s^{\prime}$. Now let $k^{\prime}$ be minimal such that $r_{k^{\prime}}^{\prime}$ lies on some 1 -skeleton geodesic $r_{k} s^{\prime}$. Consider various 1skeleton geodesics $\psi$ connecting $r_{k}$ with $r_{k^{\prime}}$. The loops $r_{m} r_{m+1} \cdots r_{k} \psi r_{k^{\prime}}^{\prime} r_{k^{\prime}-1}^{\prime} \cdots r_{m}^{\prime}$ are embedded by the choice of $m, k, k^{\prime}$. Consider a surface $S: D \rightarrow X$ of minimal area spanned on such a loop (we allow $\psi$ to vary). By minimality of the area $D$ is systolic, hence CAT(0) with respect to the standard piecewise Euclidean metric. Denote the preimages of $r_{i}, r_{i}^{\prime}, \psi$ in $D$ by $x_{i}, x_{i}^{\prime}, \alpha$ respectively. We attach to $D$ at $x_{k}, x_{k^{\prime}}^{\prime}, x_{m}=$ $x_{m}^{\prime}$ three simplicial paths $\beta, \beta^{\prime}, \zeta$ of lengths $n-k, n^{\prime}-k^{\prime}, m$ respectively and denote obtained in this way simplicial (and CAT(0)) complex by $D^{\prime}$. Denote the vertices in $D^{\prime} \backslash D$ by $x_{n}, \ldots, x_{k+1}$, by $x_{n^{\prime}}^{\prime}, \ldots, x_{k^{\prime}+1}^{\prime}$, and by $x_{0}=x_{0}^{\prime}, \ldots, x_{m-1}=x_{m-1}^{\prime}$ in $\beta, \beta^{\prime}, \zeta$ respectively.

By minimality of the area of $D$, the path $\beta \alpha \beta^{\prime-1}$ is a CAT(0) geodesic in $D^{\prime}$. Let $D_{1}, D_{2}$ be simplicial spans in $D^{\prime}$ of the unions of all 1 -skeleton geodesics from $x_{0}$ 
to $x_{n}$ and from $x_{0}^{\prime}$ to $x_{n^{\prime}}^{\prime}$, respectively. Observe that $D_{1}, D_{2}$ are convex (in CAT(0) sense) in $D^{\prime}$, hence the CAT(0) geodesics in $D^{\prime}$ from $x_{0}$ to $x_{n}$ and from $x_{0}^{\prime}$ to $x_{n^{\prime}}^{\prime}$ agree with $\operatorname{CAT}(0)$ geodesics joining those pairs of points in $D_{1}, D_{2}$, respectively. By Proposition 12.1, $\left(x_{i}\right)$ is 97-close (in $\left.D_{1}\right)$ to the CAT( 0$)$ geodesic $x_{0} x_{n}$ and $\left(x_{i}^{\prime}\right)$ is 97-close (in $D_{2}$ ) to the CAT(0) geodesic $x_{0}^{\prime} x_{n^{\prime}}^{\prime}$.

Our goal, which immediately implies Theorem 13.1 (Theorem C), is to get an estimate $\left|x_{\lfloor c n\rfloor} x_{\left\lfloor c n^{\prime}\right\rfloor}^{\prime}\right| \leq c\left|x_{n} x_{n^{\prime}}^{\prime}\right|+C$ with some universal constant $C$.

We claim that for any three consecutive vertices $v, w, u$ on $\alpha$ we have that $\left|x_{0} w\right|=$ $\left|x_{0} v\right|+1$ implies $\left|x_{0} u\right|=\left|x_{0} w\right|+1$. We prove this claim by contradiction. If $\left|x_{0} u\right|=\left|x_{0} w\right|-1$ then, by Lemma 2.8, $u, v$ are neighbors contradicting the fact that $v w u$ is a 1 -skeleton geodesic. If $\left|x_{0} u\right|=\left|x_{0} w\right|$, then by Lemma 2.8 there exists a vertex $z \in D$ in the projection of the edge $w u$ onto $B_{\left|x_{0} v\right|}\left(x_{0}\right)$. Again by Lemma 2.8, we have that $|z v| \leq 1$. Thus the defect at $w$ is at least 1 , contradicting the minimality of the area of $D$. This justifies the claim.

The claim implies that $\alpha$ is a concatenation $\alpha_{1} \alpha_{0} \alpha_{2}$, where vertices in $\alpha_{0}$ are at constant distance from $x_{0}$ and $\alpha_{1}, \alpha_{2}$ are contained in 1-skeleton geodesic rays in $D^{\prime}$ issuing from $x_{0}$. We apply Lemma 13.2 to obtain a special 1 -skeleton geodesic $\omega$ in $D^{\prime}$ connecting $x_{0}$ to $\alpha_{1} \cap \alpha_{0}$. Let $\widetilde{D}_{1}$ be the union of $\omega$ and all of the components of $D^{\prime} \backslash \omega$ containing some $x_{i}$ (ie on one "side" of $\omega$ ). Denote by $\widetilde{D}_{1}^{c}$ the union of $\omega$ with the other components of $D^{\prime} \backslash \omega$. Denote by $\omega^{\prime}$ a 1 -skeleton geodesic connecting $x_{0}$ to $\alpha_{0} \cap \alpha_{2}$ given by Lemma 13.2 applied do $\widetilde{D}_{1}^{c}$. Let $\widetilde{D}_{2}$ be the union of $\omega^{\prime}$ with the components of $\tilde{D}_{1}^{c} \backslash \omega^{\prime}$ containing some $x_{i}^{\prime}$. Denote the union of $\omega^{\prime}$ with the other components of $\tilde{D}_{1}^{c} \backslash \omega^{\prime}$ by $\tilde{D}_{0}$.

Note that, since $\widetilde{D}_{1} \subset D_{1}, \widetilde{D}_{2} \subset D_{2}$, by Lemma 13.3 we have that $\left(x_{i}\right)$ is 97-close to the CAT(0) geodesic $x_{0} x_{n}$ in $\widetilde{D}_{1}$ and $\left(x_{i}^{\prime}\right)$ is 97-close to the CAT(0) geodesic $x_{0}^{\prime} x_{\mathfrak{p}^{\prime}}^{\prime}$ in $\widetilde{D}_{2}$. Moreover, by Lemma 13.2 and Lemma 13.3, the CAT(0) geodesics in $\widetilde{D}_{0}, \widetilde{D}_{1}, \widetilde{D}_{2}$ joining the endpoints of $\omega, \omega^{\prime}$ are 1-close (in particular 97-close) to $\omega, \omega^{\prime}$, respectively. Moreover, vertices in $\alpha_{0}$ are at constant distance from $x_{0}$ in $\widetilde{D}_{0}$, and $\omega \alpha_{1}^{-1}, \omega^{\prime} \alpha_{2}$ are 1 -skeleton geodesics in $\widetilde{D}_{1}, \widetilde{D_{2}}$, respectively. Thus substituting $D^{\prime}=\widetilde{D}_{0}, \widetilde{D}_{1}, \widetilde{D}_{2}$ we have reduced the proof of our goal (up to replacing $C$ with $3 C$ ) to the following two special cases:

(i) Vertices in $\alpha$ are at a constant distance from $x_{0}$ (hence from $x_{m}$ ), or

(ii) $n^{\prime}=k^{\prime}$ and $\alpha x_{k^{\prime}}^{\prime} \cdots x_{0}^{\prime}$ is a 1 -skeleton geodesic.

Observe that it is now possible that $x_{i}=x_{i}^{\prime}$ for $i>m$. Let $m^{\prime}$ be maximal such that $x_{m^{\prime}}=x_{m^{\prime}}^{\prime}$. If $\lfloor c n\rfloor \leq m^{\prime}$ or $\left\lfloor c n^{\prime}\right\rfloor \leq m^{\prime}$, say the latter, then, since the CAT(0) geodesics 
$x_{0} x_{n}, x_{0}^{\prime} x_{n^{\prime}}^{\prime}$ in $D^{\prime}$ coincide on $x_{0} x_{m^{\prime}}$, we get that $\left|x_{\left\lfloor c n^{\prime}\right\rfloor} x_{\left\lfloor c n^{\prime}\right\rfloor}^{\prime}\right| \leq 97+97=194$, hence

$$
\begin{aligned}
\left|x_{\lfloor c n\rfloor} x_{\left\lfloor c n^{\prime}\right\rfloor}^{\prime}\right| & \leq\left|x_{\lfloor c n\rfloor} x_{\left\lfloor c n^{\prime}\right\rfloor}\right|+\left|x_{\left\lfloor c n^{\prime}\right\rfloor} x_{\left\lfloor c n^{\prime}\right\rfloor}^{\prime}\right| \\
& \leq\left|\lfloor c n\rfloor-\left\lfloor c n^{\prime}\right\rfloor\right|+194<c\left|n-n^{\prime}\right|+195 \leq c\left|x_{n} x_{n^{\prime}}^{\prime}\right|+195,
\end{aligned}
$$

as desired. So from now on we can assume that $\lfloor c n\rfloor>m^{\prime},\left\lfloor c n^{\prime}\right\rfloor>m^{\prime}$, and we can replace the component of $D^{\prime} \backslash x_{m^{\prime}}$ containing $x_{0}$ with a simplicial path of length $m^{\prime}$. Let $D$ be as before the maximal subcomplex of $D^{\prime}$ which is a topological disc.

First suppose that we are in case (i). Observe that (up to increasing $C$ by 2) we can assume that $n=k$ and $n^{\prime}=k^{\prime}$. This is because once we proved our estimate for $n=k, n^{\prime}=k^{\prime}$ we can concatenate an estimate realizing path $x_{\lfloor c k\rfloor} x_{\left\lfloor c k^{\prime}\right\rfloor}^{\prime}$ with the paths $x_{\lfloor c k\rfloor} \cdots x_{\lfloor c n\rfloor}$ and $x_{\left\lfloor c k^{\prime}\right\rfloor}^{\prime} \cdots x_{\left\lfloor c n^{\prime}\right\rfloor}^{\prime}$, obtaining a path from $x_{\lfloor c n\rfloor}$ to $x_{\left\lfloor c n^{\prime}\right\rfloor}^{\prime}$ of length

$$
\begin{aligned}
& (\lfloor c n\rfloor-\lfloor c k\rfloor)+\left|x_{\lfloor c k\rfloor} x_{\left\lfloor c k^{\prime}\right\rfloor}^{\prime}\right|+\left(\left\lfloor c n^{\prime}\right\rfloor-\left\lfloor c k^{\prime}\right\rfloor\right) \\
& <(c(n-k)+1)+\left(c\left|x_{k} x_{k^{\prime}}\right|+C\right)+\left(c\left(n^{\prime}-k^{\prime}\right)+1\right) \\
& =\left(c\left(\left|x_{n} x_{k}\right|\right)+1\right)+\left(c\left|x_{k} x_{k^{\prime}}\right|+C\right)+\left(c\left(\left|x_{n^{\prime}} x_{k^{\prime}}\right|\right)+1\right)=c\left|x_{n} x_{n^{\prime}}\right|+(C+2),
\end{aligned}
$$

as required.

We claim that $D$ is flat and the interior vertices of $\alpha$ have defect 0 . Indeed, observe that the defects at the interior vertices of $\alpha$ and at the interior vertices of $D$ are nonpositive, whereas the defect at $x_{m^{\prime}}=x_{m^{\prime}}^{\prime}$ is at most 2. Hence, by Gauss-Bonnet Lemma 7.2, it is enough to prove that the sums of the defects at the vertices of each of the paths $x_{m^{\prime}+1} \cdots x_{k}$ and $x_{m^{\prime}+1}^{\prime} \cdots x_{k^{\prime}}$ are at most 2. Suppose otherwise, without loss of generality, that the sum of the defects at the vertices of $x_{m^{\prime}+1} \cdots x_{k}$ is $\geq 3$. Denote the vertex following $x_{k}$ on $\alpha$ by $y$. Then $\left|x_{m^{\prime}} y\right| \leq\left|x_{m^{\prime}+1} x_{k}\right|$, hence $\left|x_{0} y\right|<\left|x_{0} x_{k}\right|$, which contradicts the hypothesis of case (ii). Thus we have proved the claim. In particular, $\alpha$ is contained in a straight line in $D \subset \mathbb{E}_{\Delta}^{2}$ and $k=k^{\prime}$.

Define $\eta$ to be the path in $D$ starting at $x_{\lfloor c k\rfloor}$ reaching $x_{\lfloor c k\rfloor}^{\prime}$ contained (in $D \subset \mathbb{E}_{\Delta}^{2}$ ) in a straight line parallel to $\alpha$. Let $\xi_{1}, \xi_{2}$ be CAT(0) geodesics in $D$ joining $x_{k}$ with $x_{m^{\prime}}$ and $x_{k}^{\prime}$ with $x_{m^{\prime}}^{\prime}=x_{m^{\prime}}$, respectively. Let $z_{i}=\eta \cap \xi_{i}$, for $i=1,2$. We have $\left|x_{\lfloor c k\rfloor} z_{1}\right| \leq 97$ and $\left|z_{2} x_{\lfloor c k\rfloor}^{\prime}\right| \leq 97$ (again exceptionally $|\cdot, \cdot|$ denotes the distance along the straight line). Let $m^{\prime \prime}$ be maximal such that $\xi_{1} \cap x_{m^{\prime \prime}} x_{m^{\prime \prime}}^{\prime}=\xi_{2} \cap x_{m^{\prime \prime}} x_{m^{\prime \prime}}^{\prime}$. Then for all $i \leq m^{\prime \prime}$ we have $\xi_{1} \cap x_{i} x_{i}^{\prime}=\xi_{2} \cap x_{i} x_{i}^{\prime}$. In particular, if $\lfloor c k\rfloor \leq m^{\prime \prime}$, then $z_{1}=z_{2}$ and $|\eta| \leq 194$, as desired. If $\lfloor c k\rfloor>m^{\prime \prime}$, then we apply Lemma 13.4 with $T \subset D$ the geodesic triangle with vertices $x_{k}, x_{k}^{\prime}, \xi_{1} \cap x_{m^{\prime \prime}} x_{m^{\prime \prime}}^{\prime}=\xi_{2} \cap x_{m^{\prime \prime}} x_{m^{\prime \prime}}^{\prime}$. We get that $|\eta| \leq c\left|x_{k} x_{k}^{\prime}\right|+194$, as desired. 
Now suppose that we are in case (ii). Like in case (i) (up to increasing $C$ by 1 ) we can assume that $n=k$. Since the boundary of $D$ is a union of two geodesics, by Gauss-Bonnet Lemma 7.2, $D$ is flat. Consider an embedding $D \subset \mathbb{E}_{\Delta}^{2}$ such that the layers (denoted by $L_{k}$ ) between $x_{m^{\prime}}=x_{m^{\prime}}^{\prime}$ and $x_{k}$ in $\mathbb{E}_{\Delta}^{2}$ are horizontal and $x_{i}$ are to the left from $x_{i}^{\prime}$, for $i \leq k^{\prime}$. By minimality of area, $\alpha$ is contained in a straight line in $D \subset \mathbb{E}_{\Delta}^{2}$. Like in case (i), let $\xi_{1}, \xi_{2}$ be CAT(0) geodesics in $D$ joining $x_{k}$ with $x_{m^{\prime}}$ and $x_{k^{\prime}}^{\prime}$ with $x_{m^{\prime}}^{\prime}=x_{m^{\prime}}$, respectively. Similarly like in the previous case, let $m^{\prime \prime}$ be maximal such that $\xi_{1} \cap L_{m^{\prime \prime}}=\xi_{2} \cap L_{m^{\prime \prime}}$. Denote $u=\xi_{1} \cap L_{m^{\prime \prime}}=\xi_{2} \cap L_{m^{\prime \prime}}$. By the same argument as after the choice of $m^{\prime}$, we can assume that $\left\lfloor c k^{\prime}\right\rfloor>m^{\prime \prime}$. Let $z_{1}=\xi_{1} \cap L_{\lfloor c k\rfloor}, z_{2}=\xi_{2} \cap L_{\left\lfloor c k^{\prime}\right\rfloor}$. Let $y_{1} \in L_{\lfloor c k\rfloor} \cap D$ be the vertex with minimal possible $y_{1}^{x}$ but $\geq z_{1}^{x}$. Similarly, let $y_{2} \in L_{\left\lfloor c k^{\prime}\right\rfloor} \cap D$ be the vertex with maximal possible $y_{2}^{x}$ but $\leq z_{2}^{x}$. We claim that $\left|y_{1} y_{2}\right|=\lfloor c k\rfloor-\left\lfloor c k^{\prime}\right\rfloor$.

Before we justify the claim, observe that it already implies the theorem. Indeed, the claim gives

$$
\begin{aligned}
\left|x_{\lfloor c k\rfloor} x_{\left\lfloor c k^{\prime}\right\rfloor}^{\prime}\right| & \leq\left|x_{\lfloor c k\rfloor} y_{1}\right|+\left|y_{1} y_{2}\right|+\left|y_{2} x_{\left\lfloor c k^{\prime}\right\rfloor}^{\prime}\right| \\
& \leq 97+\left(\lfloor c k\rfloor-\left\lfloor c k^{\prime}\right\rfloor\right)+97 \\
& <97+\left(c\left(k^{\prime}-k\right)+1\right)+97=c\left|x_{k} x_{k^{\prime}}^{\prime}\right|+195
\end{aligned}
$$

as desired.

Finally, let us justify the claim. We need to show that $y_{2}^{x}-y_{1}^{x} \leq\left(\lfloor c k\rfloor-\left\lfloor c k^{\prime}\right\rfloor\right) / 2$. By the choice of $m^{\prime \prime}$ we have that $z_{1}, z_{2}$ lie in the Euclidean triangle in $\mathbb{E}_{\Delta}^{2}$ with vertices $x_{k}, x_{k^{\prime}}^{\prime}, u$. Denote by $u_{1}$ (resp. $u_{2}$ ) the vertex on the edge $u x_{k}$ (resp. $u x_{k^{\prime}}^{\prime}$ ) of this triangle in $L_{\lfloor c k\rfloor}$ (resp. $L_{\left\lfloor c k^{\prime}\right\rfloor}$ ). Assume without loss of generality that $\left\lfloor c k^{\prime}\right\rfloor / k^{\prime} \geq$ $\lfloor c k\rfloor / k$. Denote then by $u_{*}$ the vertex on the edge $u x_{k}$ dividing this edge in same proportion as the proportion in which $u_{2}$ divides $u x_{k^{\prime}}^{\prime}$. By the Tales Theorem, and since $u_{1} u_{*} \subset u x_{k}$ forms with the vertical direction angle at most $30^{\circ}$, we have that

$$
\begin{aligned}
u_{2}^{x}-u_{1}^{x} & \leq\left(u_{2}^{x}-u_{*}^{x}\right)+\left(u_{*}^{x}-u_{1}^{x}\right)<c\left(x_{k}^{x}-\left(x_{k^{\prime}}^{\prime}\right)^{x}\right)+\frac{1}{2} \\
& =\frac{c k-c k^{\prime}}{2}+\frac{1}{2}<\frac{\lfloor c k\rfloor-\left\lfloor c k^{\prime}\right\rfloor}{2}+1,
\end{aligned}
$$

hence

$$
y_{2}^{x}-y_{1}^{x} \leq z_{2}^{x}-z_{1}^{x} \leq u_{2}^{x}-u_{1}^{x}<\frac{\lfloor c k\rfloor-\left\lfloor c k^{\prime}\right\rfloor}{2}+1 \text {. }
$$

Thus, since $y_{2}^{x}-y_{1}^{x}$ and $\left(\lfloor c k\rfloor-\left\lfloor c k^{\prime}\right\rfloor\right) / 2$ differ by an integer (because $y_{1}, y_{2}$ are vertices in $\mathbb{E}_{\Delta}^{2}$ ), we have $y_{2}^{x}-y_{1}^{x} \leq\left(\lfloor c k\rfloor-\left\lfloor c k^{\prime}\right\rfloor\right) / 2$, as desired. This ends the proof of the claim and of the whole theorem. 
If we followed the constants carefully, we would get that Theorem 13.1 (Theorem C) is satisfied with any $C \geq 204$.

\section{Final remarks}

In this section we state some additional results on the compactification $\bar{X}$, for which we do not provide proofs.

$E Z$-structures explored by Farrell-Lafont [16] in relation to the Novikov conjecture concern only the torsion-free group case. To get similar results (Novikov conjecture) for a group $G$ with torsion one needs to construct an appropriate compactification (which we will also call an $E Z$-structure) of a classifying space for proper $G$-actions, denoted $\underline{E} G . \underline{E} G$ is a contractible space with a proper $G$ action such that, for every finite subgroup $F$ of $G$, the set $\underline{E} G^{F} \subset \underline{E} G$ of points fixed by $F$ (the fixed point set of $F$ ) is contractible (in particular nonempty). For more details on $\underline{E} G$ see Lück [21]. Relying on the work of Przytycki [23], Chepoi-Osajda [9] proved the following.

Theorem 14.1 Let a group $G$ act geometrically by simplicial automorphisms on a systolic complex $X$. Then $X$ is a finite model for $\underline{E} G$.

We claim the following.

Claim 14.2 Let a group $G$ act geometrically by simplicial automorphisms on a systolic complex $X$. Let $\bar{X}=X \cup \partial X$. Then

(1) for every finite subgroup $F$ of $G$, the fixed point set $\bar{X}^{F}$ is contractible,

(2) for every finite subgroup $F$ of $G$, the fixed point set $X^{F}$ is dense in $\bar{X}^{F}$.

Assertion 2 is easy to prove, ie the only difficulties in proving Claim 14.2 concern assertion 1. To obtain it one has to reprove Lemma 6.2 with $\bar{X}^{F}$ in place of $\bar{X}$.

Combining Theorem 14.1, Theorem 6.3 (Theorem A), Claim 14.2, and Theorem 4.1 of Rosenthal [24], we immediately get the following.

Claim 14.3 The Novikov conjecture holds for systolic groups.

Now we turn to the question of determining our boundary in some specific cases. We have already mentioned the case of hyperbolic systolic groups in Remark 4.6. Now we consider the CAT(0) case. After making it through the second part of the article, the reader should not be surprised by the following. 
Claim 14.4 If $X$ is a two-dimensional simplicial complex, which is CAT(0) (which is equivalent with systolic in dimension two), then its compactification by the CAT(0) visual boundary is homeomorphic in a natural way with our $\bar{X}$.

For example, this implies that our boundary of a systolic Euclidean plane is a circle. The argument for Claim 14.4 is that our compactification is constructed using Euclidean geodesics in systolic complexes, which in this case are coarsely CAT(0) geodesics.

The next claim concerns the following construction, which has not yet appeared in the literature. Namely Elsner and Przytycki had developed a way to turn equivariantly any $\mathcal{V H}$-complex (see Bridson-Wise [6]) which is CAT(0) into a systolic complex (that is how they observed that the abelian product of two free groups is systolic). Although the resulting complex is usually not 2 -dimensional, the only higher dimensional simplices that appear are used to deal with branching at the vertical edges. This is why we believe that the CAT(0) visual boundary of the original $\mathcal{V H}$-complex is homeomorphic in a natural way with our boundary of the resulting systolic complex.

In particular, this would imply that there is a systolic group acting geometrically on two systolic complexes whose (our) boundaries are not homeomorphic. Namely, in the family of torus complexes defined by Croke-Kleiner [10] the complexes with $\alpha=\frac{\pi}{2}$ and $\alpha=\frac{\pi}{3}$ have universal covers with nonhomeomorphic CAT(0) visual boundaries. At the same time, there is a torus complex with $\alpha=\frac{\pi}{3}$, whose universal cover is 2dimensional systolic while there also is a torus complex with $\alpha=\frac{\pi}{2}$, whose universal cover is a $\mathcal{V H}$-complex, which is $\operatorname{CAT}(0)$.

\section{References}

[1] G Arzhantseva, MR Bridson, T Januszkiewicz, IJ Leary, A Minasyan, J Światkowski, Infinite groups with fixed point properties, Geom. Topol. 13 (2009) 1229-1263 MR2496045

[2] A Bartels, W Lück, The Borel Conjecture for hyperbolic and CAT(0)-groups arXiv: $0901.0442 \mathrm{v} 1$

[3] M Bestvina, Local homology properties of boundaries of groups, Michigan Math. J. 43 (1996) 123-139 MR1381603

[4] M Bestvina, G Mess, The boundary of negatively curved groups, J. Amer. Math. Soc. 4 (1991) 469-481 MR1096169

[5] B H Bowditch, Cut points and canonical splittings of hyperbolic groups, Acta Math. 180 (1998) 145-186 MR1638764

[6] M R Bridson, D T Wise, $\mathcal{V H}$ complexes, towers and subgroups of $F \times F$, Math. Proc. Cambridge Philos. Soc. 126 (1999) 481-497 MR1684244 
[7] G Carlsson, E K Pedersen, Controlled algebra and the Novikov conjectures for Kand L-theory, Topology 34 (1995) 731-758 MR1341817

[8] V Chepoi, Graphs of some CAT(0) complexes, Adv. in Appl. Math. 24 (2000) 125-179 MR1748966

[9] V Chepoi, D Osajda, Dismantlability of weakly systolic complexes and applications, in preparation

[10] C B Croke, B Kleiner, Spaces with nonpositive curvature and their ideal boundaries, Topology 39 (2000) 549-556 MR1746908

[11] F Dahmani, Classifying spaces and boundaries for relatively hyperbolic groups, Proc. London Math. Soc. (3) 86 (2003) 666-684 MR1974394

[12] A N Dranishnikov, On Bestvina-Mess formula, from: "Topological and asymptotic aspects of group theory”, (R Grigorchuk, M Mihalik, M Sapir, Z Šunik, editors), Contemp. Math. 394, Amer. Math. Soc. (2006) 77-85 MR2216707

[13] J Dugundji, Topology, Allyn and Bacon, Boston (1966) MR0193606

[14] T Elsner, Systolic spaces with isolated flats, submitted

[15] T Elsner, Flats and the flat torus theorem in systolic spaces, Geom. Topol. 13 (2009) 661-698 MR2469526

[16] F T Farrell, J-F Lafont, EZ-structures and topological applications, Comment. Math. Helv. 80 (2005) 103-121 MR2130569

[17] F Haglund, Complexes simpliciaux hyperboliques de grande dimension, Prepublication Orsay 71 (2003)

[18] F Haglund, J Świątkowski, Separating quasi-convex subgroups in 7-systolic groups, Groups Geom. Dyn. 2 (2008) 223-244 MR2393180

[19] T Januszkiewicz, J Świątkowski, Simplicial nonpositive curvature, Publ. Math. Inst. Hautes Études Sci. (2006) 1-85 MR2264834

[20] T Januszkiewicz, J Świątkowski, Filling invariants of systolic complexes and groups, Geom. Topol. 11 (2007) 727-758 MR2302501

[21] W Lück, Survey on classifying spaces for families of subgroups, from: "Infinite groups: geometric, combinatorial and dynamical aspects", (L Bartholdi, T CeccheriniSilberstein, T Smirnova-Nagnibeda, A Zuk, editors), Progr. Math. 248, Birkhäuser, Basel (2005) 269-322 MR2195456

[22] P Papasoglu, E Swenson, Boundaries and JSJ decompositions of CAT(0)-groups arXiv:0701618v1

[23] P Przytycki, E $G$ for systolic groups, Comment. Math. Helv. 84 (2009) 159-169 MR2466079

[24] D Rosenthal, Split injectivity of the Baum-Connes assembly map arXiv: math/0312047 
Instytut Matematyczny, Uniwersytet Wrocławski

pl Grunwaldzki 2/4, 50-384 Wrocław, Poland

Institute of Mathematics, Polish Academy of Sciences

Śniadeckich 8, 00-956 Warsaw, Poland

dosaj@math.uni.wroc.pl, pprzytyc@mimuw.edu.pl

Proposed: Wolfgang Lück

Seconded: Jean-Pierre Otal, Martin Bridson

Received: 18 August 2008

Revised: 15 July 2009 